Portland State University

PDXScholar

\title{
Synthesis and Characterization of the 2-Dimensional Transition Metal Dichalcogenides
}

Robert Browning

Portland State University

Follow this and additional works at: https://pdxscholar.library.pdx.edu/open_access_etds

Part of the Atomic, Molecular and Optical Physics Commons Let us know how access to this document benefits you.

\section{Recommended Citation}

Browning, Robert, "Synthesis and Characterization of the 2-Dimensional Transition Metal Dichalcogenides" (2017). Dissertations and Theses. Paper 3483.

https://doi.org/10.15760/etd.5367

This Dissertation is brought to you for free and open access. It has been accepted for inclusion in Dissertations and Theses by an authorized administrator of PDXScholar. Please contact us if we can make this document more accessible: pdxscholar@pdx.edu. 
Synthesis and Characterization of the 2-Dimensional Transition Metal Dichalcogenides

by

Robert Browning

A dissertation submitted in partial fulfillment of the requirements for the degree of

Doctor of Philosophy

in

Applied Physics

\author{
Dissertation Committee: \\ Raj Solanki, Chair \\ Shankar Rananavare \\ Andrew Rice \\ Tami Clare
}

Portland State University

2017 


\begin{abstract}
In the last 50 years, the semiconductor industry has been scaling the silicon transistor to achieve faster devices, lower power consumption, and improve device performance. Transistor gate dimensions have become so small that short channel effects and gate leakage have become a significant problem. To address these issues, performance enhancement techniques such as strained silicon are used to improve mobility, while new high-k gate dielectric materials replace silicon oxide to reduce gate leakage. At some point the fundamental limit of silicon will be reached and the semiconductor industry will need to find an alternate solution. The advent of graphene led to the discovery of other layered materials such as the transition metal dichalcogenides. These materials have a layered structure similar to graphene and therefore possess some of the same qualities, but unlike graphene, these materials possess sizeable bandgaps between 1-2 eV making them useful for digital electronic applications. Since initially discovered, most of the research on these films has been from mechanically exfoliated flakes, which are easily produced due to the weak van der Waals force binding the layers together. For these materials to be considered for use in mainstream semiconductor technology, methods need to be explored to grow these films uniformly over a large area.
\end{abstract}

In this research, atomic layer deposition (ALD) was employed as the growth technique used to produce large area uniform thin films of several different transition metal dichalcogenides. By optimizing the ALD growth parameters, it is possible to grow 
high quality films a few to several monolayers thick over a large area with good uniformity. This has been demonstrated and verified using several physical analytical tests such as Raman spectroscopy, photoluminescence, $\mathrm{x}$-ray photoelectron spectroscopy, $\mathrm{x}$-ray diffraction, transmission electron spectroscopy, and scanning electron microscopy, which show that these films possess the same qualities as those of the mechanically exfoliated films. Back-gated field effect transistors were created and electrical characterization was performed to determine if ALD grown films possess the same electronic properties as films produced from other methods. The tests revealed that the ALD grown films have high field effect mobility and high current on/off ratios. The $\mathrm{WSe}_{2}$ films also exhibited ambipolar electrical behavior making them a possible candidate for complementary metal-oxide semiconductor (CMOS) technology. Ab-initio density functional theory calculations were performed and compared to experimental properties of $\mathrm{MoS}_{2}$ and $\mathrm{WSe}_{2}$ films, which show that the ALD films grown in this research match theoretical predictions. The transconductance measurements from the $\mathrm{WSe}_{2}$ devices used, matched very well with the theoretical calculations, bridging the gap between experimental data and theoretical predictions. Based upon this research, ALD growth of TMD films proves to be a viable alternative for silicon based digital electronics. 


\section{Dedication}

This dissertation is dedicated to my wife who has stood by me all these years, encouraging and supporting me, and to my parents who always believed in me. 


\section{Acknowledgements}

I would like to acknowledge my advisor Prof. Raj Solanki who has been a constant source of encouragement, knowledge and support over the years. Your guidance and direction have enabled me to accomplish a goal I would never have been able to complete otherwise. I thank you for the opportunity to be able to work on such an important project which has been challenging and extremely rewarding. 


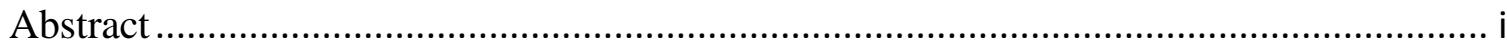

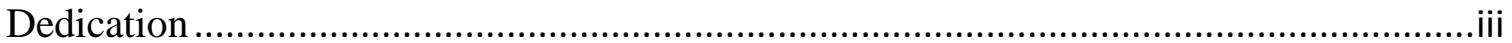

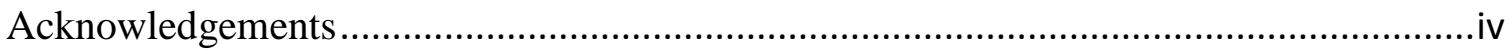

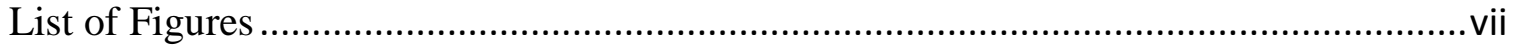

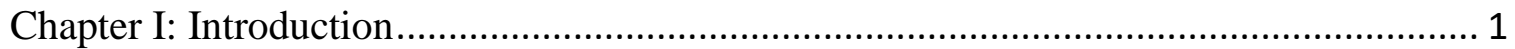

1.1 Device miniaturization and the need for TMD films ...................................... 1

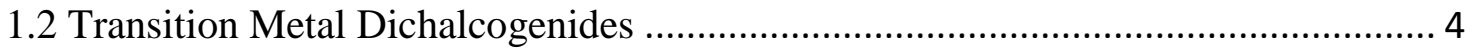

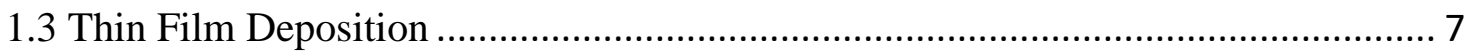

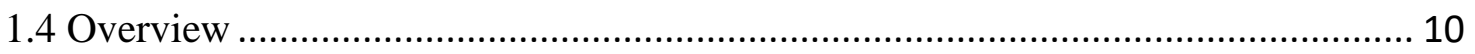

Chapter II: Synthesis and Physical Characterization .................................................... 12

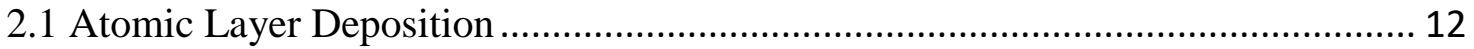

2.2 Film Characterization: Structural Techniques and Elemental Analysis................. 16

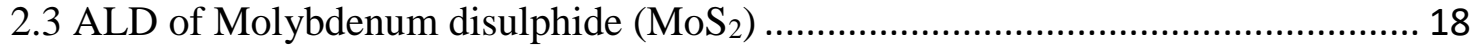

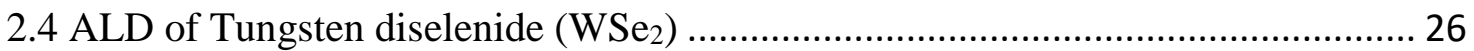

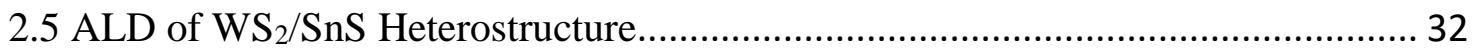

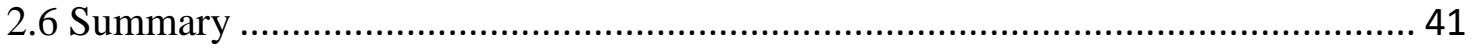

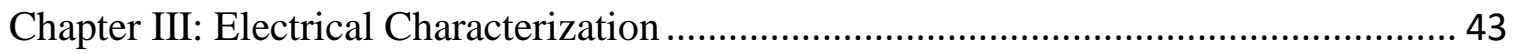

3.1 Device Fabrication and Measurement Techniques .......................................... 43

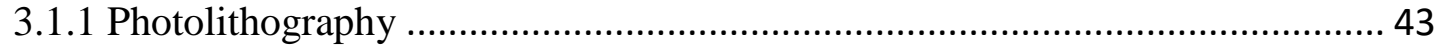

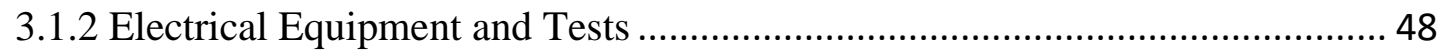

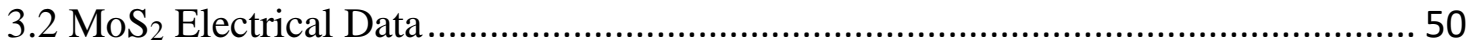

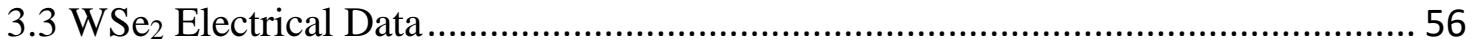

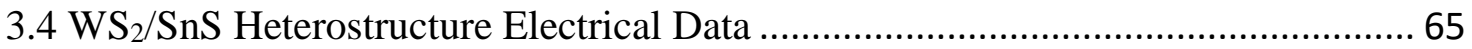

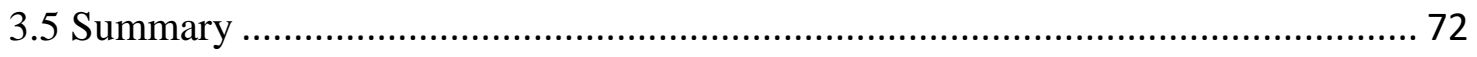

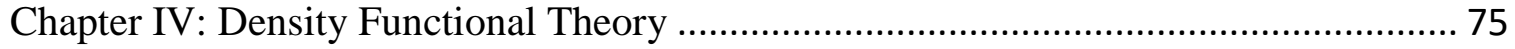

4.1 Introduction and Theoretical Background........................................................ 75

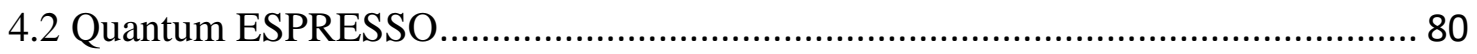

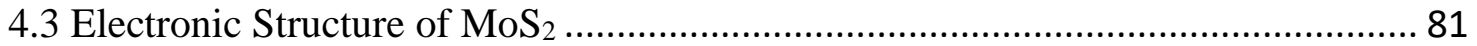




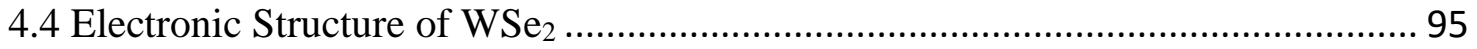

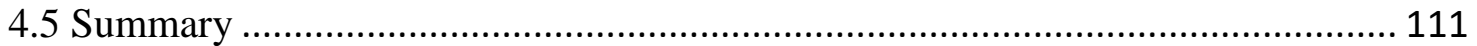

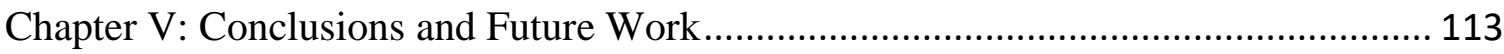

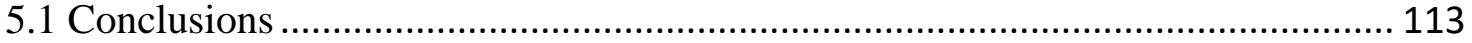

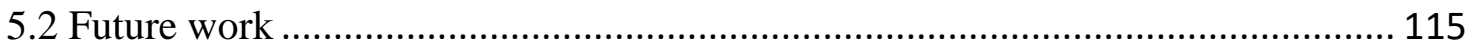

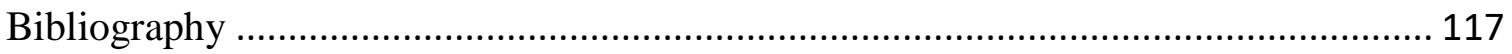




\section{List of Figures}

Figure 1.1: Classic MOSFET scaling first described by Dennard in 1974 IEEE [1] ......... 1 Figure 1.2: Side (a) and top (b) view of two layer $2 \mathrm{H}$ phase $\mathrm{MoS}_{2}$. The black box outlines the $\mathrm{MoS}_{2}$ unit cell 5

Figure 1.3: Optical micrograph (a) and AFM image (b) of $\mathrm{MoS}_{2}$ flakes produced by mechanical exfoliation [20]. Reprinted with permission from ACS Nano. Copyright 2010 American Chemical Society. 8

Figure 1.4: STM image of MBE grown $\mathrm{MoSe}_{2}$ film [22]........................................ 9

Figure 1.5: $\mathrm{MoS}_{2}$ microribbons grown via electrochemical synthesis. The time denotes number of hours $\mathrm{MoO}_{2}$ was exposed to $\mathrm{H}_{2} \mathrm{~S}$ at $800{ }^{\circ} \mathrm{C}$ to create $\mathrm{MoS}_{2}$ ribbons [12]. Reprinted with permission from Nano Letters. Copyright 2004 American Chemical Society

Figure 1.6: Optical microscopy image (a) and AFM image (b) of CVD grown monolayer $\mathrm{WSe}_{2}$ [23]. Reprinted with permission from ACS Nano. Copyright 2015 American Chemical Society. 10

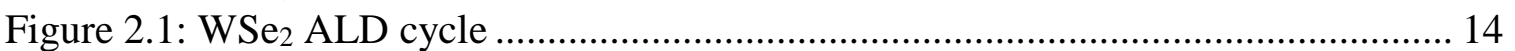

Figure 2.2: Microchemistry F-120 ALD Reactor .................................................... 15

Figure 2.3: Raman spectrum of bilayer $\mathrm{MoS}_{2}$ film on silicon oxide on silicon substrate

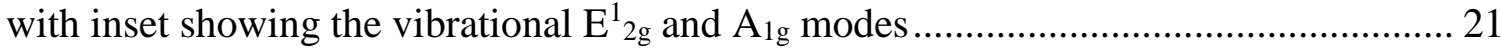
Figure 2.4: Scanning electron micrograph (SEM) of ALD deposited $\mathrm{MoS}_{2}$ film [28].... 22

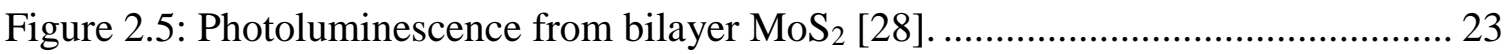
Figure 2.6: (a) XPS spectrum of $\mathrm{MoS}_{2}$ film showing Mo3d doublet [28]. (b) XPS spectrum of $\mathrm{MoS}_{2}$ with $\mathrm{S} 2 \mathrm{p}$ doublet. ................................................................... 25 Figure 2.7: X-ray diffraction spectrum of bilayer $\mathrm{MoS}_{2}$ thin film on a silicon oxide coated

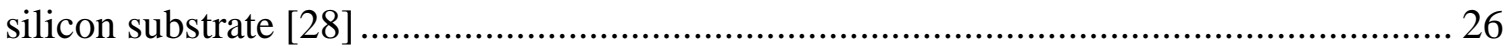

Figure 2.8: Side (a) and top (b) view of two layer $2 \mathrm{H}$ phase of $\mathrm{WSe}_{2} \ldots \ldots \ldots \ldots \ldots \ldots \ldots \ldots . . . . . . . . . . . . . .27$ Figure 2.9: Raman spectrum of $\mathrm{WSe}_{2}$ produced with an excitation wavelength of $532 \mathrm{~nm}$

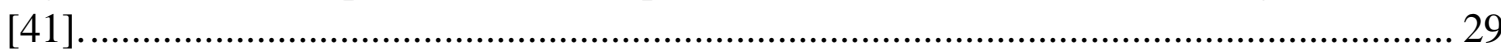

Figure 2.10: Cross-sectional TEM of a few layered thick WSe $\mathrm{W}_{2}$ film [41] ..................... 30 Figure 2.11: Top view TEM scan of $\mathrm{WSe}_{2}[41]$......................................................... 31

Figure 2.12: XPS spectra for binding energies of (a) W and (b) Se in ALD WSe 2 [41].. 32

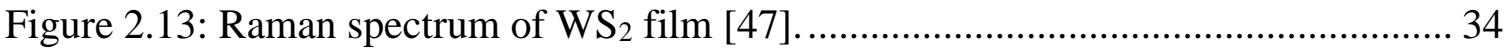

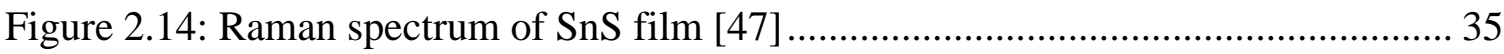

Figure 2.15: Raman spectrum of the $\mathrm{WS}_{2} / \mathrm{SnS}$ heterostructure [47] ............................ 36 Figure 2.16: Cross-sectional TEM of 3 alternating sets of $\mathrm{WS}_{2} / \mathrm{SnS}$ layers, starting with

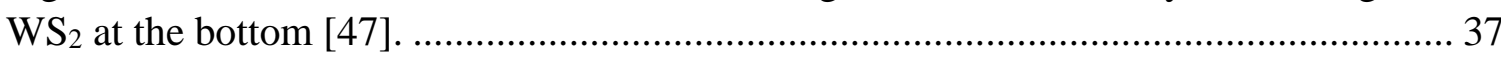

Figure 2.17: Cross-sectional TEM micrograph of the $\mathrm{WS}_{2} / \mathrm{SnS}$ interface [47].............. 38 
Figure 2.18: (a) FFT of $\mathrm{SnS}$ part of the film stack. Lattices are shown on the FFT. Blue is the $\mathrm{c}^{*}$ axis of $\mathrm{SnS}$ and red is the $\mathrm{b}^{*}$ lattice of $\mathrm{SnS}$. (b) FFT of the $\mathrm{WS}_{2}$ part of the film stack [47]....

Figure 2.19: FFT of the interface section of Fig. 2.17 [47]. The green lattice corresponds to the $\mathrm{WS}_{2}$ interlayer spacing. The blue $\mathrm{c}^{*}$ axis of $\mathrm{SnS}$, red $\mathrm{b}^{*}$ axis of $\mathrm{SnS}$.................. 40

Figure 3.1: OAI Hybralign 200 Contact Aligner .............................................................. 45

Figure 3.2: Close up view of completed wafer, patterned with metal contacts showing the individual transistors and TLM structures. ................................................................... 47

Figure 3.3: $\mathrm{WSe}_{2}$ wafer patterned with metal deposition..........................48

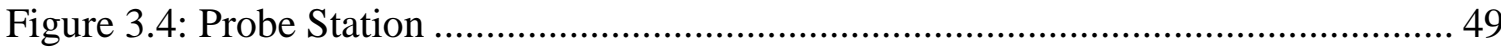

Figure 3.5: HP 4145B Semiconductor Parameter Analyzer ......................................... 50

Figure 3.6: Drain current $\left(\mathrm{I}_{\mathrm{d}}\right)$ vs. drain voltage $\left(\mathrm{V}_{\mathrm{d}}\right)$ of $\mathrm{MoS}_{2}$ bilayer film...................... 52

Figure 3.7: $\log \mathrm{I}_{\mathrm{d}}$ vs. $\mathrm{V}_{\mathrm{g}}$ plot of the back-gated $\mathrm{MoS}_{2}$ transistor [28] ............................ 53

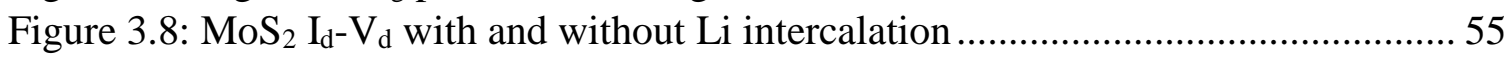

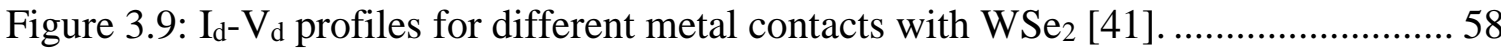

Figure 3.10: $\mathrm{I}_{\mathrm{d}}-\mathrm{V}_{\mathrm{d}}$ profile of $\mathrm{Pd}$ and Ni contacts before and after post-metallization anneal. $I_{d}$ increases for the same $V_{d}$ indicating lower resistance post metal anneal........ 59

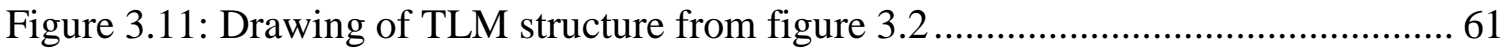

Figure 3.12: Measured resistance between the Au contacts of the TLM structure for WSe $\mathrm{W}_{2}$

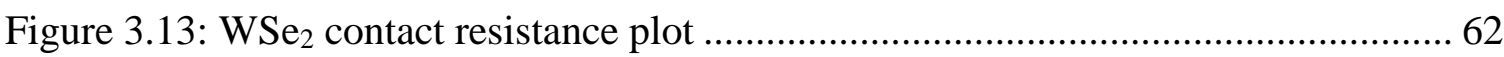

Figure 3.14: Plot of the field effect current vs. the back-gate voltage showing ambipolar

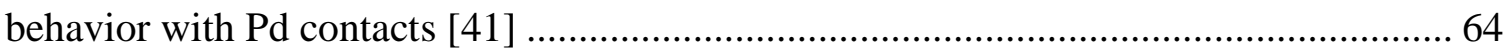

Figure 3.15: Plot of the field effect current vs. the back-gate voltage with Ni contacts... 65

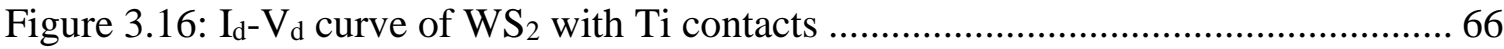

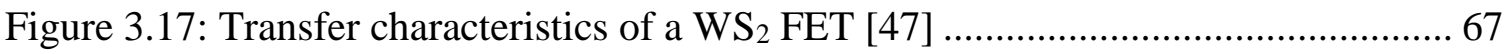

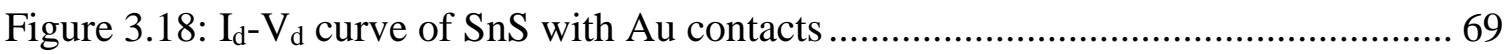

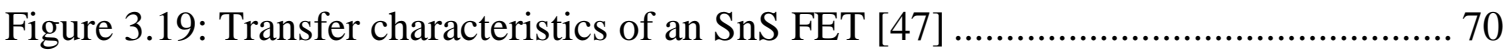

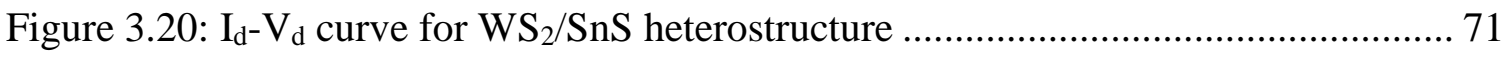

Figure 3.21: Transfer characteristics of the heterojunction FET [47] ............................ 72

Figure 4.1: Schematic representation of the self-consistent loop of the Kohn-Sham

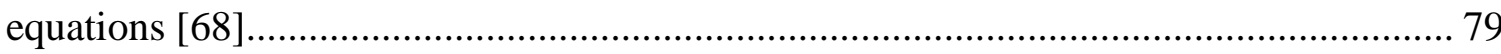

Figure 4.2: (a) Monolayer $\mathrm{MoS}_{2}$ (b) 2H polytype (c) 3R polytype [74] ....................... 82

Figure 4.3: The first Brillouin zone of hexagonal unit cell and the IBZ ......................... 83

Figure 4.4: Cutoff energy and k-point convergence study. Inset shows small difference

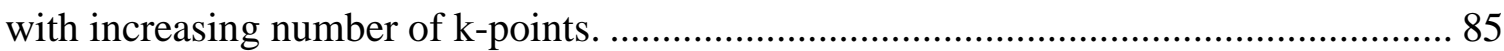

Figure 4.5: Electronic band structure of bulk, bilayer, and monolayer $\mathrm{MoS}_{2} \ldots \ldots \ldots \ldots \ldots . . . . .88$ Figure 4.6: Calculated projected density of states (PDOS) of bulk, bilayer, and monolayer $\mathrm{MoS}_{2}$ for $\mathrm{Mo}(4 \mathrm{~d})$ and $\mathrm{S}(3 \mathrm{p})$ states 89

Figure 4.7: Calculated band structure and orbital projected density of states (PDOS) for bilayer $\mathrm{MoS}_{2}$ 
Figure 4.8: Wannier functions of the Mo $4 \mathrm{~d}$ orbitals for bilayer $\mathrm{MoS}_{2}$. Yellow and bluegreen isosurfaces correspond to positive and negative charge respectively. 92 Figure 4.9: Wannier functions of the S 3p orbitals for bilayer $\mathrm{MoS}_{2}$. Yellow and bluegreen isosurfaces correspond to positive and negative charge respectively................... 93 Figure 4.10: $\mathrm{MoS}_{2}$ bilayer film theoretical quantum conductance ................................ 94 Figure 4.11: $\mathrm{MoS}_{2}$ bilayer band structure, conductance, and density of states ............... 95

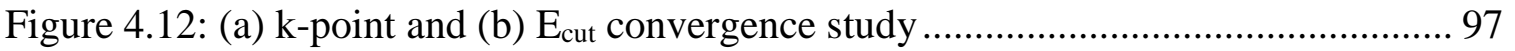
Figure 4.13: Electronic band structure of bulk and monolayer $\mathrm{WSe}_{2}$........................... 99 Figure 4.14: Calculated projected density of states (PDOS) of bulk and monolayer $\mathrm{WSe}_{2}$ for $\mathrm{W}(4 \mathrm{~d})$ and $\mathrm{Se}(3 \mathrm{p})$ states 100 Figure 4.15: Calculated band structure and orbital projected density of states (PDOS) for bulk $\mathrm{WSe}_{2}$ 101

Figure 4.16: Wannier functions for the 5d orbitals for bulk WSe2. Yellow and blue-green isosurfaces correspond to positive and negative charge respectively. 103 Figure 4.17: Wannier functions for the $4 \mathrm{p}$ orbitals for bulk $\mathrm{WSe}_{2}$. Yellow and blue-green isosurfaces correspond to positive and negative charge respectively.... 104 Figure 4.18: Comparison of experimental and theoretical conductance of bulk WSe 2 film. 105

Figure 4.19: Theoretical bulk conductance adjusted for Pd contact resistance as compared to experimental conductance from $\mathrm{WSe}_{2}$ sample. 107

Figure 4.20: Theoretical bulk conductance adjusted for Ni contact resistance as compared to experimental conductance from $\mathrm{WSe}_{2}$ sample 108

Figure 4.21: $\mathrm{WSe}_{2}$ bulk film band structure, conductance, and density for states 110 
Chapter I: Introduction

\subsection{Device miniaturization and the need for TMD films}

For over 50 years the semiconductor industry has endeavored to scale down the dimensions of silicon based electronics to reduce power consumption, improve device performance, and lower cost. A large part of the success of the MOS (metal-oxide semiconductor) transistor is because it can be scaled to increasingly smaller dimensions, which results in its higher performance. The ability to improve performance consistently while decreasing power consumption has made CMOS (complementary metal-oxide semiconductor) architecture the dominant technology for integrated circuits, especially logic circuits. The scaling of the MOS transistor has been the primary factor driving improvements in microprocessor performance. In 1974 Robert Dennard [1] published a paper listing the scaling enablers for miniaturizing silicon based electronics.

TABLE I

Scaling Results for Crrcuit Performance

\begin{tabular}{ll}
\hline Device or Circuit Parameter & Scaling Factor \\
\hline Device dimension $t_{\mathrm{ox}}, L, W$ & $1 / \kappa$ \\
Doping concentration $N_{a}$ & $\kappa$ \\
Voltage $V$ & $1 / \kappa$ \\
Current $I$ & $1 / \kappa$ \\
Capacitance $\epsilon A / t$ & $1 / \kappa$ \\
Delay time/circuit $V C / I$ & $1 / \kappa$ \\
Power dissipation/circuit $V I$ & $1 / \kappa^{2}$ \\
Power density $V I / A$ & 1 \\
\hline
\end{tabular}

Figure 1.1: Classic MOSFET scaling first described by Dennard in 1974 IEEE [1] 
He suggested that scaling channel oxide thickness, transistor gate length, and transistor channel width by a constant factor of $1 / k$ ( $k$ is a scaling constant) would provide a delay improvement of $1 / k$ at a constant power factor, thereby increasing transistor speed. To better understand this speed enhancement, we need to understand what determines the speed of the metal-oxide semiconductor field effect transistor (MOSFET). The main performance metric for transistor speed is gate delay (amount of time for a change on the input of the gate to cause a change in the output), which is defined as $C_{\text {load }} V_{D} / I_{D}$, where $C_{\text {load }}$ is the load capacitance, $V_{D}$ is the source to drain, or supply, voltage and $I_{D}$ is the drain or drive current. The formula for the drive current in the linear region is [2];

$$
I_{D}=\frac{Z \mu_{e f f} C_{o x}^{\prime}}{L}\left[\left(V_{G S}-V_{T}\right)-\frac{V_{D}}{2}\right] V_{D}
$$

for $V_{G S} \geq V_{T}, V_{D}<V_{D, S A T}$. Drive current in the saturation region is;

$$
I_{D}=\frac{Z \mu_{e f f} C_{o x}^{\prime}}{2 L}\left(V_{G S}-V_{T}\right)^{2}
$$

for $V_{G S} \geq V_{T}, V_{D} \geq V_{D, S A T}$. $L$ is the channel length, $Z$ is the channel width ( $\perp$ to $L$ ), $\mu_{\text {eff }}$ is the carrier mobility under the gate, $V_{G S}$ is the gate voltage, $V_{T}$ is the threshold voltage and $C^{\prime}{ }_{o x}$ is the gate capacitance per unit area, $\epsilon_{\mathrm{ox}} / \mathrm{t}_{\mathrm{ox}}$, where $\epsilon_{\mathrm{ox}}$ is the gate oxide electric permittivity and $t_{0 x}$ is the gate oxide thickness. To increase the speed of the MOSFET, we need to reduce the gate delay, which generally has been done by decreasing $L$ or $t_{o x}$ which in turn increases the drive current and reduces the gate delay, $C_{\text {load }} V_{D} / I_{D}$, to increase transistor speed: $\uparrow I_{D}=\frac{Z \mu_{e f f} C_{o x}^{\prime} \uparrow}{L \downarrow}\left[\left(V_{G S}-V_{T}\right)-\frac{V_{D}}{2}\right] V_{D}$. This decrease in $L$ and $t_{o x}$ 
explains why transistor size scaling had been the driving force behind device performance improvement until about 15 years ago. The desire for faster transistors started a decade long era of scaling that was seen in the 1990's. Each new generation of process technology was expected to reduce minimum feature size by approximately $0.7 \mathrm{x}$ $(k \sim 1.4)$. A $0.7 x$ reduction in linear features size was considered to be the right amount of reduction for a new process generation since it provided $\sim 2 \mathrm{x}$ increase in transistor density. In conjunction with the density increase, Dennard's scaling assumed that channel doping concentration could be continually increased to enable shorter channel lengths with the appropriate $V_{T}$. Unfortunately, increasing channel doping concentration too high can cause carrier mobility and performance to degrade due to increased impurity scattering, and source and drain junction leakage to increase due to direct tunneling. Junction leakage is a limiter for ultra-low power integrated circuits. Gate oxide thickness reduction has also slowed due to leakage from tunneling. In Intel's $65 \mathrm{~nm}$ process, the gate oxide is about $1.2 \mathrm{~nm}$ or about 5 atoms thick, which is generally considered to be the limit that $\mathrm{SiO}_{2}$ can be scaled. The general guideline for devices with ultra-thin body is that the body thickness be about $1 / 3$ the gate length to maintain effective electrostatic control of the channel by the gate [3]. For sub-5nm gate lengths, this corresponds to channel materials with only about two atomic layers in thickness. Channel gate length is getting so small that quantum tunneling and gate leakage will be too high to continue with silicon. Unfortunately, this "classic" scaling held up until about 15 years ago, when problems such as increased gate leakage caused by extremely thin gate oxide, degraded carrier mobility, short channel effects, device stability, etc., caused classic scaling to 
reach its limit. Since that time, classic scaling has been replaced with "equivalent scaling" techniques like strained silicon, high- $/$ metal gate, and tri-gate transistor architecture. The search for materials that can perform as well or better than silicon is an ongoing pursuit that must continue if we are to continue to reduce device dimensions.

\subsection{Transition Metal Dichalcogenides}

In 2004 it was discovered that atomically thin layers of graphite, commonly referred to as graphene, could easily be produced using mechanical exfoliation [4], because of the weak van der Waals force between the layers of the highly oriented graphite. The discovery of graphene attracted significant attention because of its extremely high electrical and thermal conductivity, high carrier mobility and molecular barrier properties. Unfortunately, large area graphene has no bandgap, resulting in small or no current on/off ratios in its field effect transistors (FETs), and is therefore not a suitable material for digital electronic applications. Very small band gaps have been introduced by shaping graphene, but this increases the complexity of manufacturability and significantly reduces the mobility. These issues make it very difficult to build logic circuits from graphene, therefore trying to produce semiconducting graphene requires that we look for other suitable alternatives for silicon based electronics. The discovery of graphene did, however, reveal some interesting electrical, mechanical, and optical properties of several other 2-dimensional (2D) layered crystals, such as the transition metal dichalcogenides. Transition metal dichalcogenides (TMDs) are a class of materials with the chemical formula $\mathrm{MX}_{2}$, where $\mathrm{M}$ is the transition metal from group IV (Ti, $\mathrm{Zr}$, Hf, etc.), group V (Ta, V, etc.), or group VI (Mo, W, etc.) and X is the chalcogenide (S, 
$\mathrm{Se}$, or $\mathrm{Te}$ ) atom. The metal atom is sandwiched in between two layers of chalcogenide atoms in a covalent bond. The adjacent layers are held together by weak van der Waals force interaction to form the bulk crystal in a variety of polytypes, which vary in stacking orders and metal atom coordination (Fig. 1.2). The TMDs can be semiconductors (eg. $\mathrm{MoS}_{2}, \mathrm{WSe}_{2}, \mathrm{WS}_{2}, \mathrm{SnS}_{2}$ ), semimetals (eg. $\mathrm{WTe}_{2}, \mathrm{TiSe}_{2}$ ), metals (eg. $\mathrm{NbS}_{2}, \mathrm{VS}_{2}$ ), and superconductors (e.g., $\mathrm{NbSe}_{2}, \mathrm{VSe}_{2}$ ).
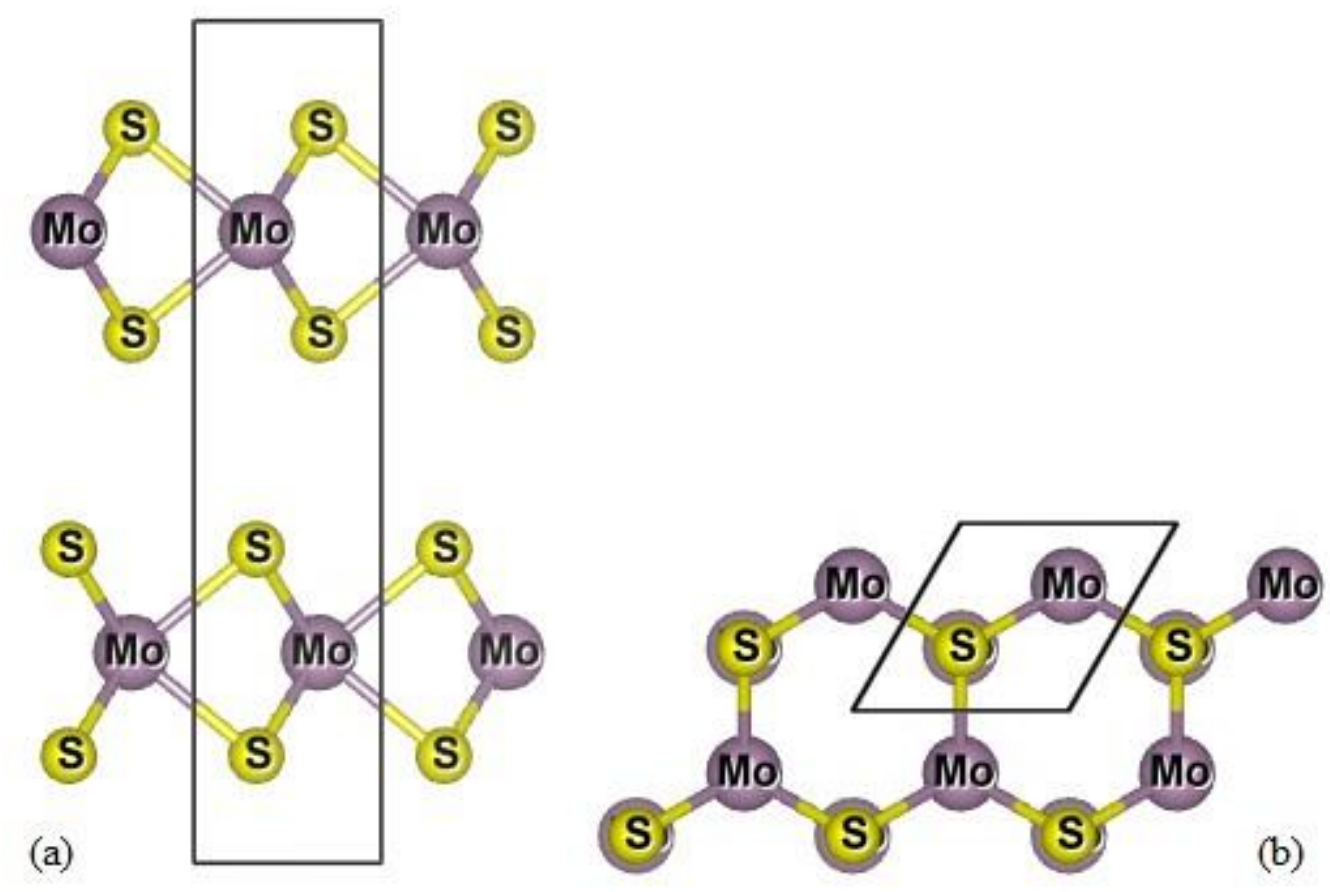

Figure 1.2: Side (a) and top (b) view of two layer $2 \mathrm{H}$ phase $\mathrm{MoS}_{2}$. The black box outlines the $\mathrm{MoS}_{2}$ unit cell.

The overall symmetry is hexagonal or rhombohedral, and the metal atoms have octahedral or trigonal prismatic coordination. The TMDs show a wide range of optical, electronic, mechanical, chemical, and thermal properties. Several of the TMDs also 
display a bandgap of between 1-2 eV making them promising materials for FETs. Some of the interesting electrical properties of transistors made from these materials show large $I_{\text {on }} / I_{\text {off }}$ ratios, low leakage current, high field effect mobility, large current carrying capacity, and a high degree of immunity to short channel effects. The high mobility of charge carriers is due to the strong covalent bonding of atoms within the layers, while the weak van der Waals bonding results in intrinsically low density of surface traps. These create pristine surfaces free of dangling bonds which reduce roughness scattering that leads to higher mobilities. A few of the TMDs show a transition from indirect bandgap in the bulk to direct bandgap in the monolayer. $\mathrm{MoS}_{2}$ has a bulk indirect bandgap of about $1.2 \mathrm{eV}$ and increases to about $1.9 \mathrm{eV}$ [5] in single layer whereas $\mathrm{WSe}_{2}$ indirect bandgap is about $1.3 \mathrm{eV}$ with a direct bandgap of about $1.6 \mathrm{eV}[6]$ at the single layer. The direct bandgap for $\mathrm{MoS}_{2}$ and $\mathrm{WSe}_{2}$ also results in photoluminescence at the monolayer which opens the possibility of optoelectronic applications.

The TMDs are promising channel materials for semiconductor logic device application, however there are some challenges to using TMDs as channel materials in MOSFETs. To date there is no suitable method for implanting these $2 \mathrm{D}$ materials due to the nature of the ultra-thin body. Theoretical work predicts partial Fermi level pinning at the interface of these materials and the metal contacts which leads to Schottky barriers [7]. To reduce the contact resistance, one would need to heavily dope the semiconductor to enable tunneling or choose a high (low) work function metal for valence (conduction) band conduction. Since the films are generally intrinsically p-type or n-type, and the 
films cannot be doped, choosing the appropriate contact metal to create Ohmic contacts can be challenging.

Another challenge is developing an adequate technique to deposit a few layer thick films of high enough quality to make them a viable gate channel material. Many of the transition metal dichalcogenides have common crystal structures that are composed of stacked layers which interact via weak van der Waals force between the adjacent layers, similar to graphene. Formation of stable thin crystals with thicknesses down to a monolayer is easily achieved using mechanical exfoliation as was done with graphene. The process of mechanical exfoliation is sufficient to create small area, ultra-thin layers for early research into the properties of the TMD films. However, depositing stable, thin, uniform films is a necessary next step in the further development of the TMDs as a viable channel material.

\subsection{Thin Film Deposition}

To date these films have been produced using a wide range of methods, including chemical vapor deposition [8-11], electrochemical synthesis [12], molecular beam epitaxy (MBE) [13], and various forms of exfoliation [14-19]. Because TMDs are layered materials like graphene, the first approach to creating monolayer thin films has been mechanical exfoliation. Mechanical exfoliation has been the mainstay of early research of the TMDs because of the relative ease of producing few to single monolayers of the film. Mechanical exfoliation (also known as "scotch tape method") typically results in flakes of varying sizes and thicknesses (Fig. 1.3) and has no large area uniformity. Mechanical 
exfoliation also suffers from possible contamination, issues with reproducibility and the ability to scale up.

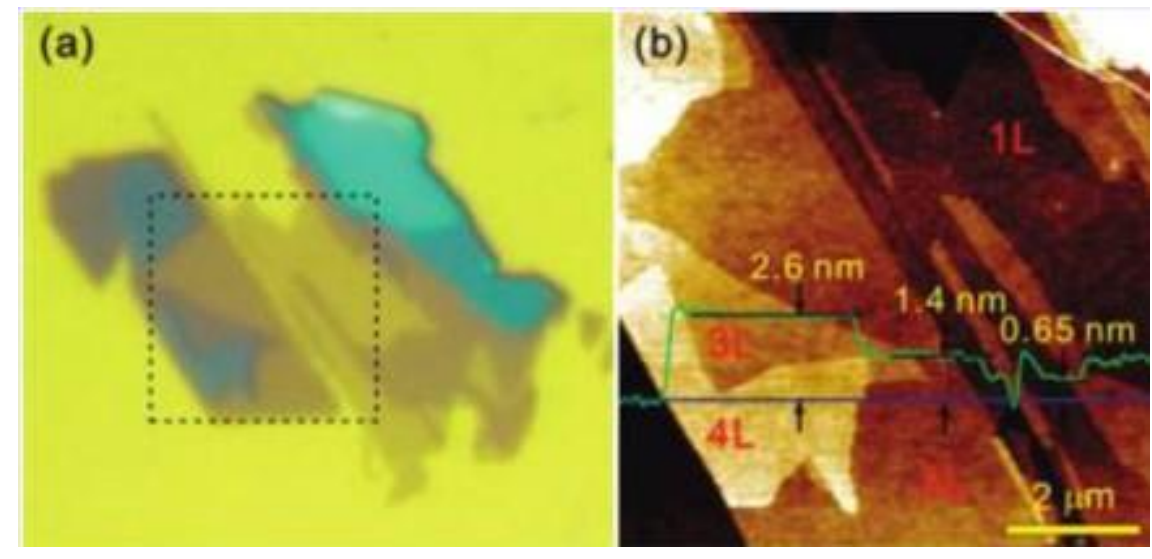

Figure 1.3: Optical micrograph (a) and AFM image (b) of $M o S_{2}$ flakes produced by mechanical exfoliation [20]. Reprinted with permission from ACS Nano. Copyright 2010 American Chemical Society.

Chemical exfoliation can produce small area thin sheets, but the chemicals are highly toxic and exothermic. Using thin film deposition methods, small area (5 $\mathrm{mm}$ x 2 $\mathrm{mm}) \mathrm{MoSe}_{2}$ films have been grown using MBE (Fig. 1.4), however larger area films suffer from domain boundary defects [21, 22] that cause mid-gap states and the process is slow and expensive. 


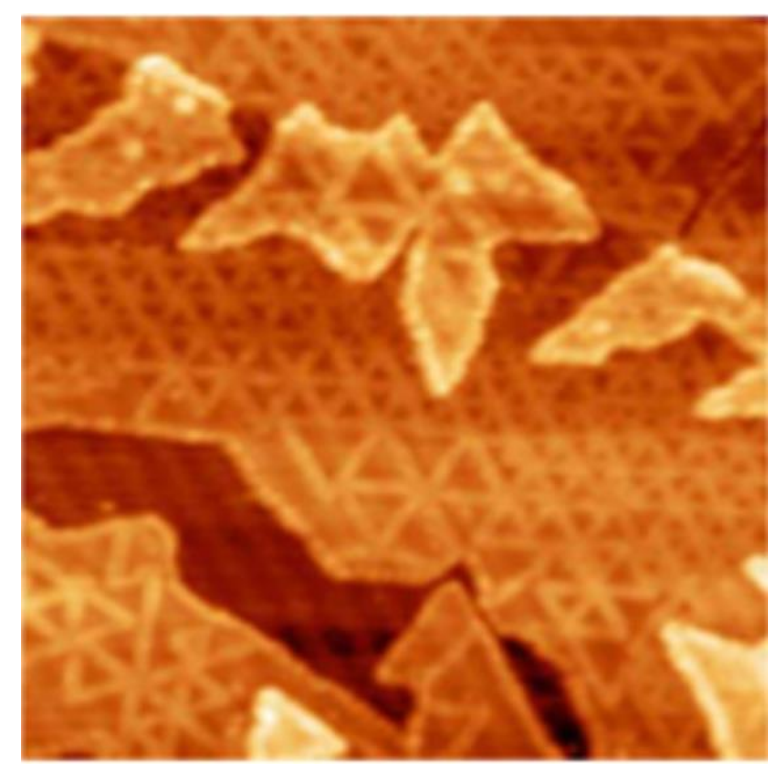

Figure 1.4: STM image of MBE grown $\mathrm{MoSe}_{2}$ film [22]

Another approach has been to produce micro-ribbons using electrochemical synthesis (Fig. 1.5). This process cannot grow large area thin films and requires high temperature $\left(800-950{ }^{\circ} \mathrm{C}\right)$ annealing for an extended time to synthesize the required film. One of the most common thin film deposition methods for advanced semiconductor technology is chemical vapor deposition (CVD), which is a bottom up approach where the reactants are delivered to a heated substrate in a gas phase reaction. The CVD process has only been able to create small area films and usually requires high temperatures to achieve these. The morphology of the films consists of platelets or plate-like crystals (Fig. 1.6) which generally have poor crystallinity. Large area uniform growth of single layer TMDs using CVD remains a challenge. Precise control of few atoms thick layered 
films over a large area is difficult, therefore an alternate method is required if we are to achieve the atomically thin films necessary.

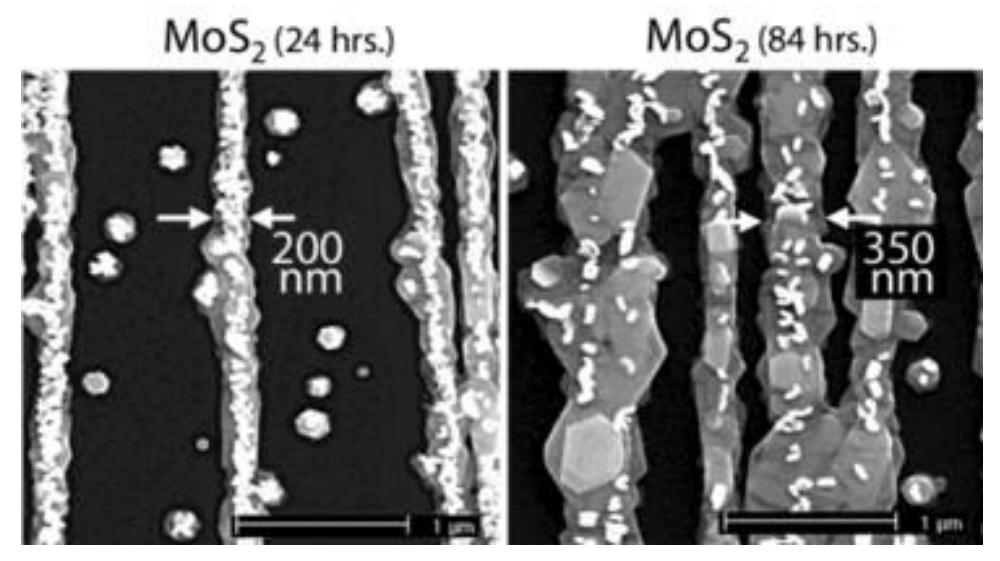

Figure 1.5: $\mathrm{MoS}_{2}$ microribbons grown via electrochemical synthesis. The time denotes number of hours $\mathrm{MoO}_{2}$ was exposed to $\mathrm{H}_{2} \mathrm{~S}$ at $800{ }^{\circ} \mathrm{C}$ to create $\mathrm{MoS}_{2}$ ribbons [12]. Reprinted with permission from Nano Letters. Copyright 2004 American Chemical Society.
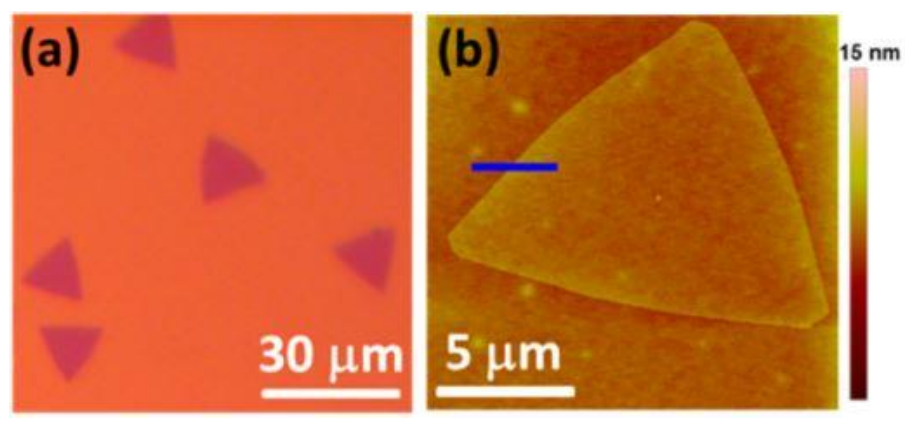

Figure 1.6: Optical microscopy image (a) and AFM image (b) of CVD grown monolayer $W_{\mathrm{Se}_{2}}$ [23]. Reprinted with permission from ACS Nano. Copyright 2015 American Chemical Society.

\subsection{Overview}


The goal of this work is to show that TMD films can be grown uniformly over a large area using atomic layer deposition, and to exhibit the unique properties these films possess. The work is divided into three areas of focus. Chapter two will discuss the film growth process of several TMD films using atomic layer deposition, and the physical properties of the materials will be examined using several characterization methods. Chapter three details the electrical characterization of these films by means of back-gated field effect transistors, including the patterning process used to create them. Chapter four is a theoretical analysis of $\mathrm{MoS}_{2}$ and $\mathrm{WSe}_{2}$ films using density functional theory. The theoretical data is then compared to the experimental data from chapters two and three so that we may bridge the gap between theory and experimental data. Finally, in chapter five, the data from chapters two, three and four is summarized. 


\section{Chapter II: Synthesis and Physical Characterization}

\subsection{Atomic Layer Deposition}

Atomic layer deposition (ALD) was introduced in 1974 by Dr. Tuomo Suntola in Finland while trying to improve the quality of $\mathrm{ZnS}$ films used in electroluminescent flat panel displays. Initially, ALD was not very well received due the fact that the film growth is a very slow process and at the time film thicknesses were far too great to make ALD useful. Continually decreasing device dimensions, and structures with high aspect ratio features that are used in advanced semiconductor technology, have increased interest towards this technique since the mid 1990's. Today it is the main deposition method used in advanced gate dielectrics, as well as other areas where very thin uniform films are required including features with high aspect ratios. No other thin film deposition technique can match the conformality achieved by ALD on high aspect ratio structures. ALD is therefore a potentially advantageous method for depositing or growing atomically thin TMD films uniformly over a large area.

ALD is a monolayer stepwise growth process where the reactants, or precursors, are alternately injected into the growth area, and following each reaction the excess species and by-products are purged out. It is unique in that the process is a selfterminating gas-solid reaction instead of a gas-gas reaction that most other CVD processes use. ALD uses many of the same chemistries that CVD uses, but CVD is done in a dynamic flow situation, where ALD uses discrete steps. As a result, high quality films are grown over large areas by sequential surface reactions which enable precise 
control of the film composition and thickness, therefore this approach is ideal for growth of layered materials. The basic ALD process first introduces a precursor gas onto the substrate surface. A chemisorption process takes place, which is a type of adsorption that is driven by a chemical reaction at the surface. This new reaction creates a new chemical species that covalently bonds to the substrate surface. The limited number of surface sites ensures that reactions can only deposit a finite number of species, creating the selfterminating process. This is also very beneficial in that an exact precursor dosage is not required, since the surface saturation limits the reaction. The only requirement is that there are enough precursor molecules to completely cover the substrate surface. After this reaction, the by-products are purged out with an inert gas (nitrogen in our case). The second precursor is then introduced which reacts with the first chemisorbed precursor which is then followed by another nitrogen purge, which completes the process and creates one monolayer of the final film. It should be noted that although the growth of one monolayer per cycle is used to describe the process, the growth per cycle is usually less than this. When the surface is saturated with the chemisorbed species, the growth per cycle is determined by factors such as the reactants used, the ALD process temperature, and sometimes the substrate material. Steric hindrance of the ligands will cause saturation during the chemisorption process if the limited number of bonding sites does not cause it. Steric hindrance is when the chemisorbed ligands of the new species blocks part of the surface from being accessible to the precursor and so the surface is considered being full. Figure 2.1 shows a flow diagram as an example of how a single layer of $\mathrm{WSe}_{2}$ is created using $\mathrm{WCl}_{6}$ and $\mathrm{H}_{2} \mathrm{Se}$ precursors as the reactants. Using this process, we have 
demonstrated growth of several of the 2D films, including $\mathrm{MoS}_{2}, \mathrm{WS}_{2}, \mathrm{SnS}$ and $\mathrm{WSe}_{2}$, over a large area $(5 \mathrm{~cm} \times 5 \mathrm{~cm})$.

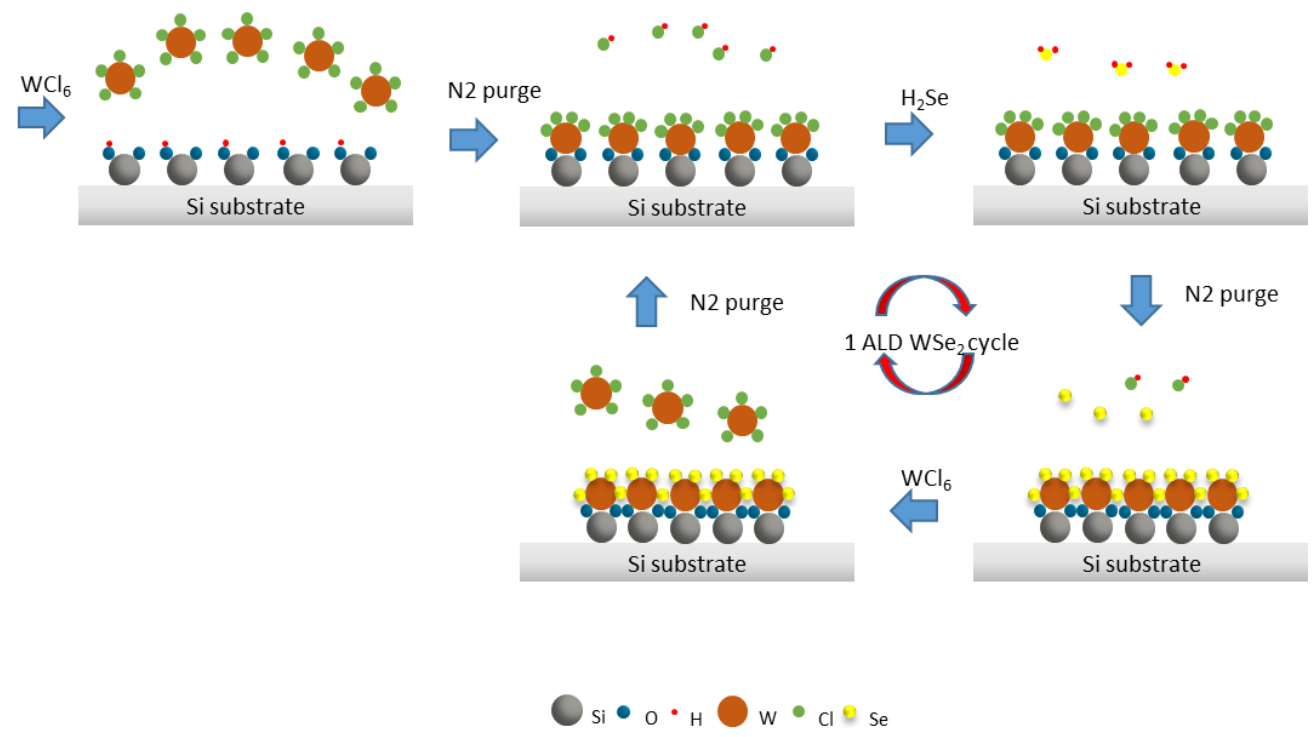

Figure 2.1: $\mathrm{WSe}_{2}$ ALD cycle

The self-limiting nature of the reactions allows the process to create thin films with atomic level control and with a high degree of uniformity and conformity, making it one of the most promising thin film deposition techniques for nanostructures, patterned substrates, and high aspect ratio features. Another characteristic of ALD is the ability to deposit films at low temperatures, which is beneficial for processes where low thermal budget is required. Depending on the film, ALD can deposit films at temperatures as low as $100{ }^{\circ} \mathrm{C}$ which has been demonstrated with $\mathrm{Al}_{2} \mathrm{O}_{3}$ and $\mathrm{ZrO}_{2}$ [24], although most of the TMD films are grown at higher temperatures to produce crystalline films of appropriate phase. 
All films used in this research were grown in a Microchemistry F-120 ALD reactor (Fig. 2.2), which can hold two $5 \mathrm{~cm}$ x $5 \mathrm{~cm}$ substrates per run. The F-120 reactor was the first commercial ALD reactor [25] and was created for fast cycling, which allowed for short pulse widths in our film growth parameters. The films typical growth temperatures ranged from $330^{\circ} \mathrm{C}$ to $450{ }^{\circ} \mathrm{C}$, based upon the film being grown. The substrates consisted of either $\mathrm{n}$ - or p-type silicon wafers coated with silicon oxide ranging in thicknesses from $30 \mathrm{~nm}$ to $320 \mathrm{~nm}$. The parameters used in the growth process for each film type are explained later in this chapter.

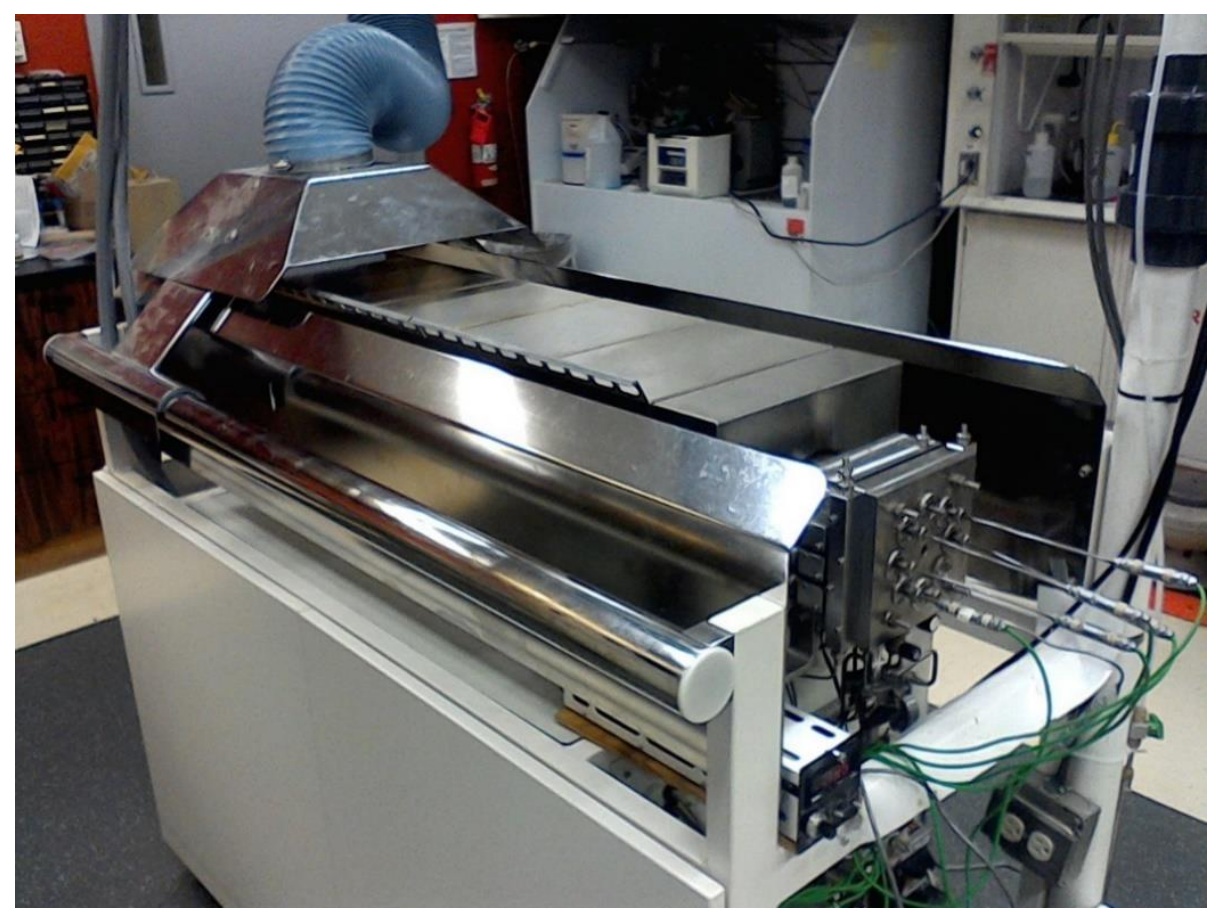

Figure 2.2: Microchemistry F-120 ALD Reactor 


\subsection{Film Characterization: Structural Techniques and Elemental Analysis}

Optimum parameters for growth of the TMD films were determined by characterizing the films with respect to their composition, crystalline phase, etc. Several physical methods for characterizing the TMD films such as XPS, XRD, SEM, TEM, photoluminescence, and Raman spectroscopy were used to determine the quality of the films. All the film characterization techniques used in this research will be briefly discussed, although not all of them were employed for every film.

Raman spectroscopy was one of the primary characterization tools used for structural characterization. It was used to observe specific materials based on their vibrational, rotational, and other low frequency modes, as well as their crystalline phase. Raman spectroscopy is an optical technique based on inelastic scattering of monochromatic light, usually from a laser source. Photons of the laser light are absorbed by the film and then re-emitted. The frequency of the re-emitted photons is shifted up or down in comparison with original monochromatic frequency, which is called the Raman effect. It was the primary characterization method used to optimize the ALD parameters for growth of $\mathrm{MoS}_{2}, \mathrm{WS}_{2}, \mathrm{SnS}, \mathrm{WS}_{2} / \mathrm{SnS}$ heterostructures and $\mathrm{WSe}_{2}$ films. Raman spectroscopy is also useful for determination of film thickness of some $2 \mathrm{D}$ chalcogenides because the phonon frequencies are sensitive to the thicknesses of these films.

Photoluminescence (PL) is a non-contact non-destructive method of probing the electronic structure of a material. The energy distribution from photoluminescence is measured and analyzed to determine properties of the material such as its band gap, using 
the formula; $\Delta E=\frac{1240 \mathrm{eV} \cdot \mathrm{nm}}{\lambda}$, where $\lambda$ is the wavelength of the emitted photons and $\Delta E$ is the energy band gap. For our $\mathrm{MoS}_{2}$ sample, when laser light of wavelength $532 \mathrm{~nm}$ was directed onto the film surface, it was absorbed, leading to photo-excitation of the electrons. The photo-excitation causes electrons to jump to a higher energy state and release photons as they relax and return to a lower energy state. The photons were measured with a $0.3 \mathrm{~m}$ spectrometer. PL could not be used in cases where there was little or no photo-excitation occurring, as in the case of the thicker samples of $\mathrm{MoS}_{2}$ or $\mathrm{WSe}_{2}$ (indirect band gaps). $\mathrm{MoS}_{2}$ does show some PL for bilayer films and strong PL for monolayer films.

Transmission electron microscopy (TEM) is a technique where a beam of electrons is transmitted through an ultra-thin specimen (film), interacting with the film as the beam passes through it. An image is formed from the electrons diffracted through the film. This image is magnified and focused onto an imaging device. TEM can produce images down to a single row of atoms and is therefore extremely useful for determining the number of layers in a film. TEM was also used to determine the internal structure and composition of our films.

X-ray photoelectron spectroscopy (XPS) is a surface chemical analysis technique that can be used to analyze the surface chemistry of a material. It measures the elemental composition to determine the empirical formula, chemical state, and electronic state of elements that were present within the material. XPS spectra for a few materials were obtained by irradiating a material with a beam of x-rays while simultaneously measuring 
the kinetic energy and number of electrons that escaped from the top to a depth of about $10 \mathrm{~nm}$ of the material being analyzed. XPS is very useful for TMD film analysis to determine the proper phase and composition of films since there are several different compositions that can be formed depending upon the growth parameters.

X-ray diffraction (XRD) is a non-destructive technique used to identify crystalline phases and orientation. XRD can determine structural properties such as lattice parameters (interplanar spacing), strain, grain size, epitaxy, phase composition, and preferred orientation. XRD can be used to measure thickness of thin films and multilayers and determine atomic arrangement. XRD was used to determine the crystalline property of $\mathrm{MoS}_{2}$ bilayer film in this research.

\subsection{ALD of Molybdenum disulphide $\left(\mathrm{MoS}_{2}\right)$}

Molybdenum disulphide $\left(\mathrm{MoS}_{2}\right)$ is the most studied of the 2D layered materials. It has a hexagonal honeycomb structure like graphene and therefore exhibits similar physical properties. The monolayer is composed of a single layer of molybdenum atoms sandwiched between two layers of sulfur atoms and coordinated through covalent bonds in a trigonal prismatic arrangement. Fig 1.2 shows a single layer of $\mathrm{MoS}_{2}$ in a $2 \mathrm{H}$ phase or polytype. As a bulk film, $\mathrm{MoS}_{2}$ is an indirect bandgap semiconductor, however monolayers of this film are interesting because they have a direct bandgap of about 1.8 eV [26] making $\mathrm{MoS}_{2}$ a good candidate for optoelectronics.

The ability to grow $\mathrm{MoS}_{2}$ (as well as the other TMDs) using ALD comes from the fact that the reactants (precursors) are readily available and can facilitate the appropriate 
growth pathway. The reactants must also be volatile enough to be in a gas phase with moderate heating and should not decompose before they have reached and reacted with the sample surface. The surface reaction must also be quick and irreversible for fast growth. The $\mathrm{MoS}_{2}$ films in this research were grown with temperatures ranging from 330 ${ }^{\circ} \mathrm{C}$ to $450{ }^{\circ} \mathrm{C}$. With temperatures ranging from $350{ }^{\circ} \mathrm{C}$ to $400{ }^{\circ} \mathrm{C}$, the films were smooth and continuous and growth was linear with the number of cycles. At a temperature of 390 ${ }^{\circ} \mathrm{C}$ the film growth rate was $0.087 \mathrm{~nm} /$ cycle. The growth per ALD cycle is much smaller than one $\mathrm{MoS}_{2}$ monolayer $(\sim 6.5 \AA)$. This discrepancy is because ALD growth is dependent on surface species and surface chemistry which can cause a limited number of reactive surface sites. The surface chemistry is not required to always yield a monolayer of growth for every ALD cycle. Several films were grown, but the thickest films were about $9 \mathrm{~nm}$ after 100 cycles. The substrates used were n-type silicon wafers with about $320 \mathrm{~nm}$ of silicon oxide grown as an electrical insulating layer. The precursors used for $\mathrm{MoS}_{2}$ were $\mathrm{MoCl}_{5}$ and $\mathrm{H}_{2} \mathrm{~S}$ (9\% Ar balance). The $\mathrm{MoCl}_{5}$ source temperature was set to $105^{\circ} \mathrm{C}$ and the carrier gas $\left(\mathrm{N}_{2}\right)$ flow rate was set to $250 \mathrm{sccm}$ and a reactor pressure of 1 mbar. The pulse sequence for each cycle was as follows; $\mathrm{MoCl}_{5}$ pulse width was $0.8 \mathrm{~s}$ with an $\mathrm{N}_{2}$ purge of $1.0 \mathrm{~s}$, followed by $\mathrm{H}_{2} \mathrm{~S}$ pulse of $1.2 \mathrm{~s}$ and then $\mathrm{N}_{2}$ purge of $1.0 \mathrm{~s}$. No further processing of the film was performed, although it should be noted that other post processing methods such as sulfur annealing have been performed by others to try and improve crystallinity [27].

After the films were grown, they were analyzed by Raman spectroscopy using $532 \mathrm{~nm}$ wavelength with laser power of $1 \mathrm{~mW}$. The Raman peaks were weak for films 
grown at $350{ }^{\circ} \mathrm{C}$, but for the films grown at $390{ }^{\circ} \mathrm{C}$ the peaks were sharp and distinct (Fig. 2.3). Figure 2.3 shows a Raman spectrum of one sample grown with 15 cycles $(\sim 1.3 \mathrm{~nm}$ thick), exhibiting an in-plane mode at $379.0 \mathrm{~cm}^{-1}$ and out-of-plane mode at $401.2 \mathrm{~cm}^{-1}$. The separation of the in-plane $\left(\mathrm{E}_{2 \mathrm{~g}}^{1}\right)$ and out-of-plane $\left(\mathrm{A}_{1 \mathrm{~g}}\right)$ vibrational modes is $\sim 22 \mathrm{~cm}^{-1}$ [28], indicating a bilayer (two $\mathrm{MoS}_{2}$ layers) film [29]. The bilayer growth was also confirmed using atomic force microscopy (AFM) that measured a thickness of $1.3 \mathrm{~nm}$. The separation in peaks of the $\mathrm{E}_{2 \mathrm{~g}}^{1}$ and $\mathrm{A}_{1 \mathrm{~g}}$ vibrational modes is one of the unique and quick methods of determining the thickness of $\mathrm{MoS}_{2}$ films. It has been shown that this separation is smaller when the films are thinner down to $\sim 18 \mathrm{~cm}^{-1}$ for the monolayer and $\sim 26 \mathrm{~cm}^{-1}$ for bulk films [30]. Using a classical model for coupled harmonic oscillator, the two vibrational modes are expected to increase as additional layers are added because the interlayer van der Waal interactions increase the effective restoring force acting on the atoms. This separation has been explained with first principles based force constant model that the frequencies between the two modes are due to the increased force constant between Mo and S at the surface and decreasing thickness. The effect of increased force 
constant for the $\mathrm{E}^{1}{ }_{2 \mathrm{~g}}$ mode is larger than the effect of decreasing thickness, explaining the anomalous blue shift [31] (frequency increase).

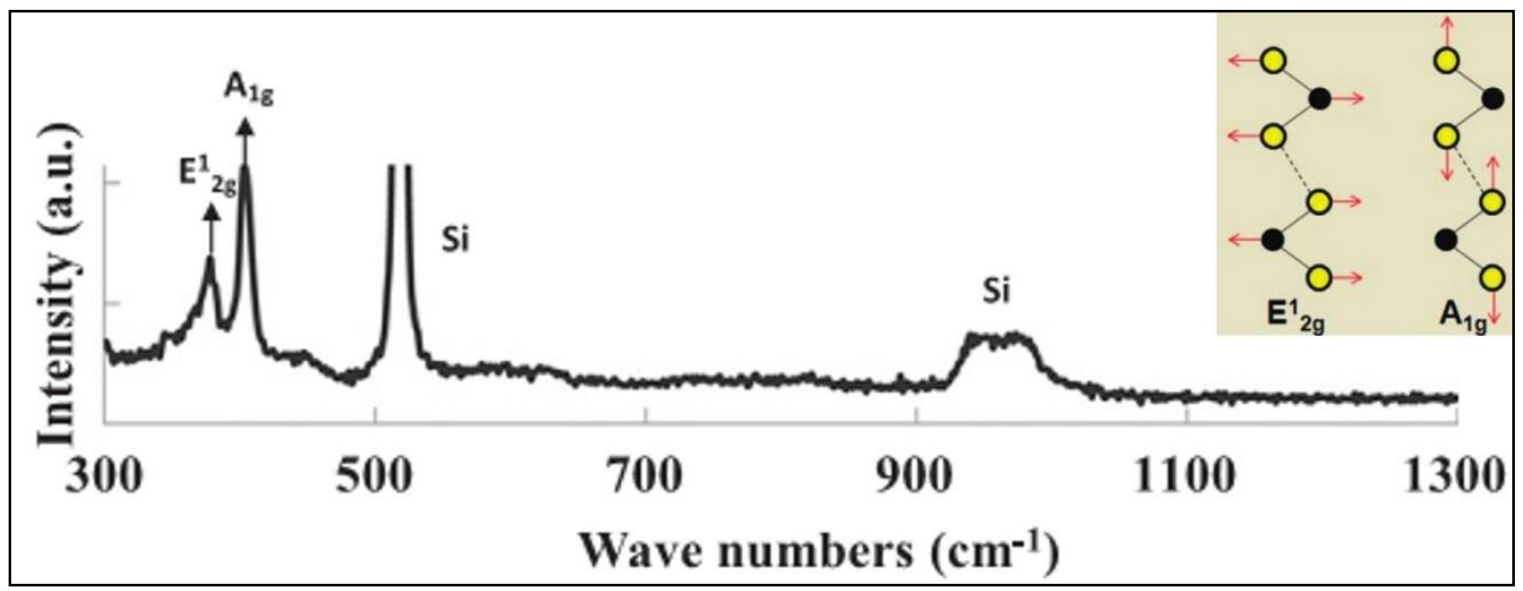

Figure 2.3: Raman spectrum of bilayer $\mathrm{MoS}_{2}$ film on silicon oxide on silicon substrate with inset showing the vibrational $E_{2 g}^{1}$ and $A_{1 g}$ modes [28].

Another test performed with Raman spectroscopy was a parallelization test, to confirm that the films were grown parallel to the substrate surface. The polarization of the incoming light was set while rotating the analyzed light. The samples tested showed the $\mathrm{A}_{1 \mathrm{~g}}$ peaks were nearly eliminated, because the phonon modes are perpendicular to the film, compared to the $\mathrm{E}^{1}{ }_{2 \mathrm{~g}}$ peaks which are in-plane oscillations, thus confirming that the film growth was parallel to the film surface.

The film uniformity was checked by measuring multiple areas of the sample using Raman spectroscopy with no discernible difference in the vibrational modes of the scans, indicating a uniform film. This was further tested with AFM and scanning electron 
microscope (SEM), which showed no step changes and the SEM image (figure 2.4) was smooth and featureless.

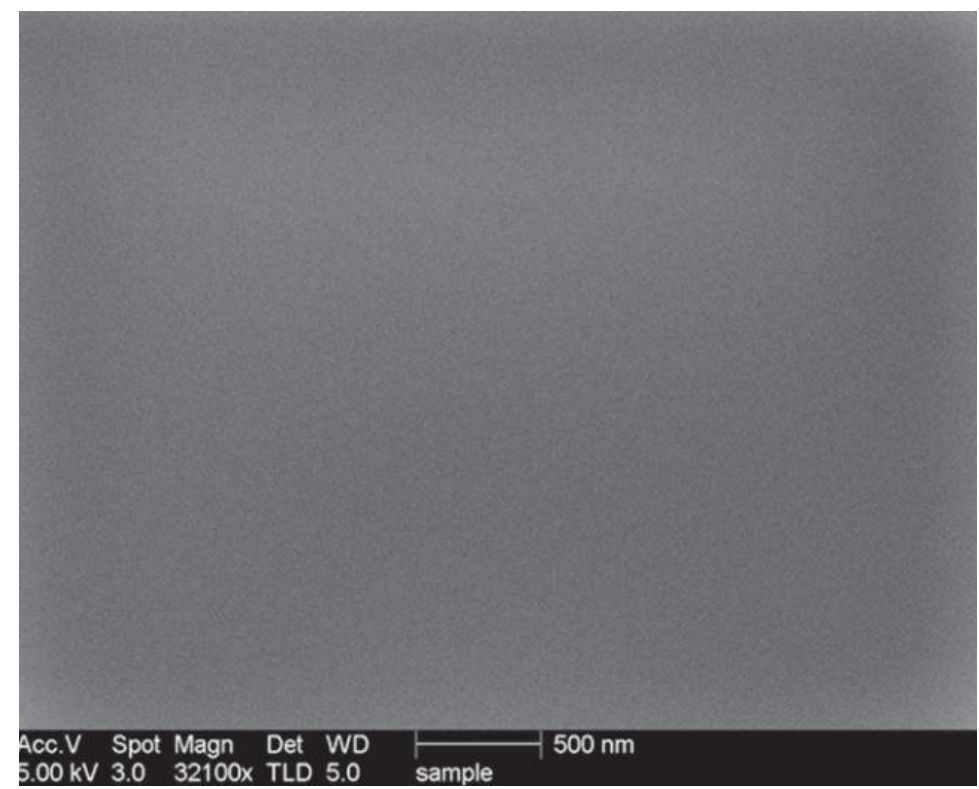

Figure 2.4: Scanning electron micrograph (SEM) of ALD deposited $\mathrm{MoS}_{2}$ film [28]

Bulk $\mathrm{MoS}_{2}$ is an indirect bandgap semiconductor with an energy gap of about 1.2 $\mathrm{eV}$ [32], but when the thickness is reduced to a single atomic layer, the bandgap becomes direct with an energy gap of about $1.9 \mathrm{eV}$ due to quantum confinement $[32,33]$. Bright photoluminescence can be seen from monolayers of $\mathrm{MoS}_{2}$ and surprisingly strong $\mathrm{PL}$ peaks can be detected from even a few layers thick films. PL was measured from the $\mathrm{MoS}_{2}$ bilayer sample, whose Raman spectrum is shown in figure 2.3, using an excitation wavelength of $532 \mathrm{~nm}$ with a $0.3 \mathrm{~m}$ spectrometer. There are two strong peaks (Fig. 2.5), one which is from the monolayer at about $680 \mathrm{~nm}(\sim 1.82 \mathrm{eV})$ and the second peak at 
about $775 \mathrm{~nm}(1.6 \mathrm{eV})$ is from the bilayer. The bilayer peak at $775 \mathrm{~nm}$ is attributed to indirect gap luminescence and the onset of photoconductivity, which is consistent with experimental work performed by others [33].

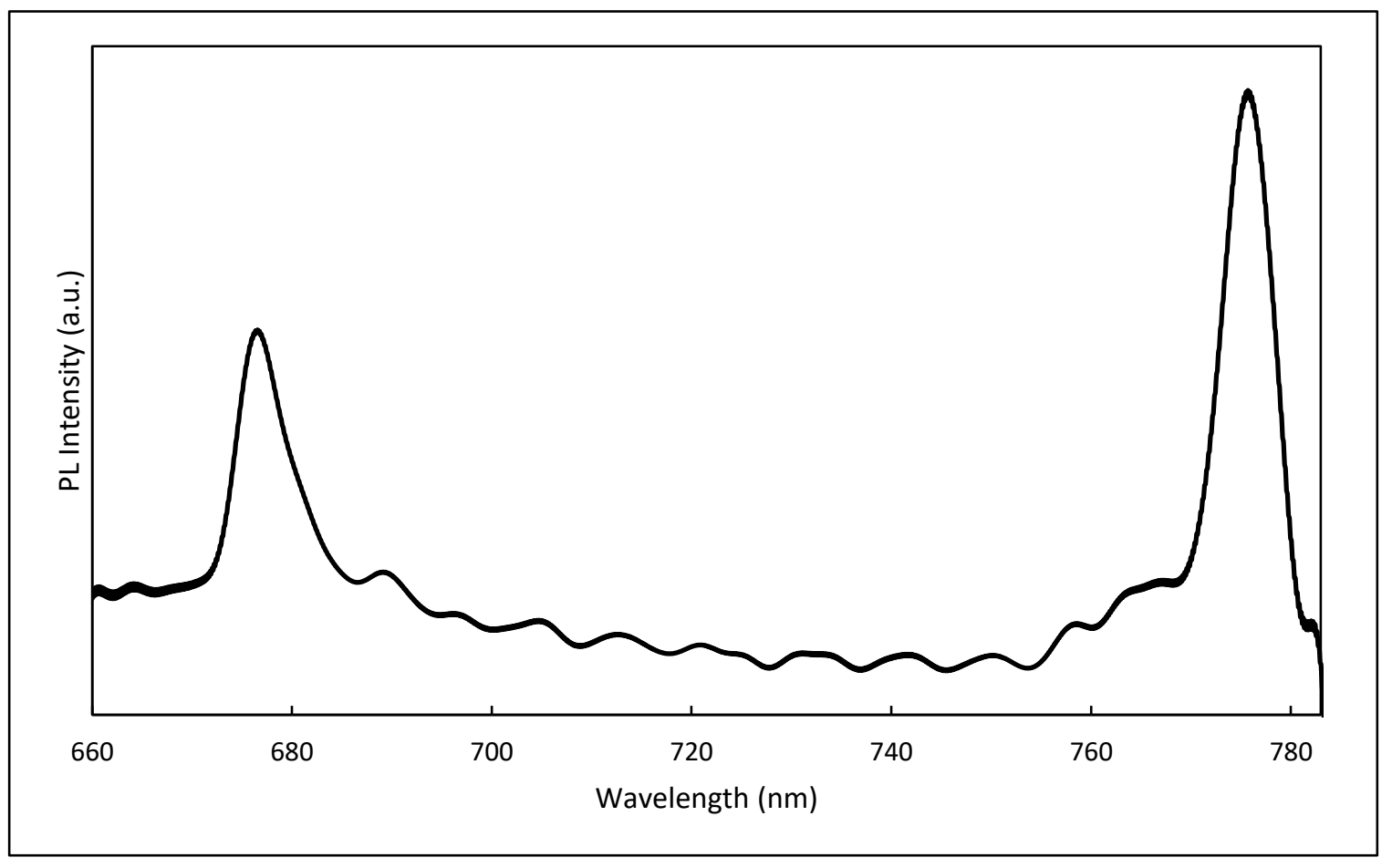

Figure 2.5: Photoluminescence from bilayer $\mathrm{MoS}_{2}$ [28].

Chemical analysis of the $\mathrm{MoS}_{2}$ bilayer film was performed using XPS. The spectrum for molybdenum and sulfur binding energies is shown in figure 2.6. The peaks at 229.9 and $233.1 \mathrm{eV}$ are attributed to the doublet Mo3d $\mathrm{d}_{5 / 2}$ and Mo3d $\mathrm{d}_{3 / 2}$ respectively, while the sulfur the peak at $227.14 \mathrm{eV}$ is attributed to the $\mathrm{S} 2 \mathrm{~s}$ binding energy, as seen in figure 2.6(a). In figure 2.6(b) the sulfur $2 \mathrm{p}$ binding energies are shown. The peaks observed at 161.4 and $160.3 \mathrm{eV}$ are attributed to the $\mathrm{S} 2 \mathrm{p}_{1 / 2}$ and $\mathrm{S} 2 \mathrm{p}_{3 / 2}$ orbitals of divalent 
sulfide ions $\left(\mathrm{S}^{2-}\right)$. The separation between the two $\mathrm{p}$ and the two $\mathrm{d}$ peaks is due to spin orbit splitting. The XPS data was calibrated against standard carbon signature, and all binding energy peaks were obtained after peak fitting with a corresponding chi-squared fit of 0.53 and are consistent with those reported for $\mathrm{MoS}_{2}$ crystals $[34,35]$. There was a small trace of chlorine from the $\mathrm{MoCl}_{5}$ precursor whose signal was too weak to determine the atomic concentration. There was also no detection of molybdenum oxide peaks. 

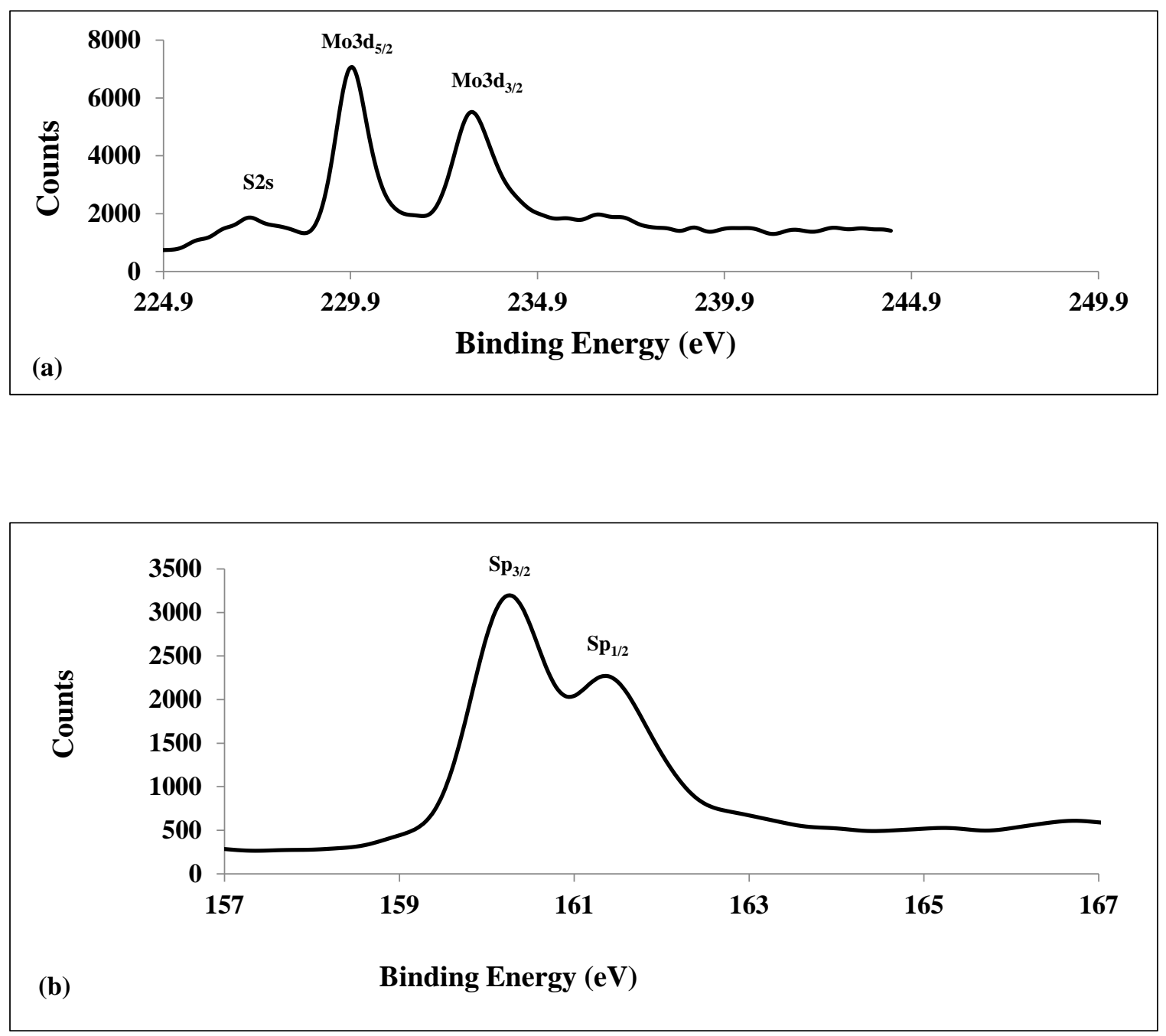

Figure 2.6: (a) XPS spectrum of MoS 2 film showing Mo3d doublet [28]. (b) XPS spectrum of $\mathrm{MoS}_{2}$ with $\mathrm{S} 2 \mathrm{p}$ doublet.

To determine the crystalline property of this bilayer film, x-ray diffraction (XRD) was performed using a very slow $(20 \mathrm{~h})$ scan rate at a grazing angle of $0.5^{\circ}$ with respect to the sample. The wide peak centered at about $13^{\circ}$ in figure 2.7 is attributed to the scattering from the 002 basal plane of the $\mathrm{MoS}_{2}$ film [36]. The broadening of the peak is 
due to the very thin film. The second peak at $21^{\circ}$ is from the underlying amorphous silica layer of the substrate [37].

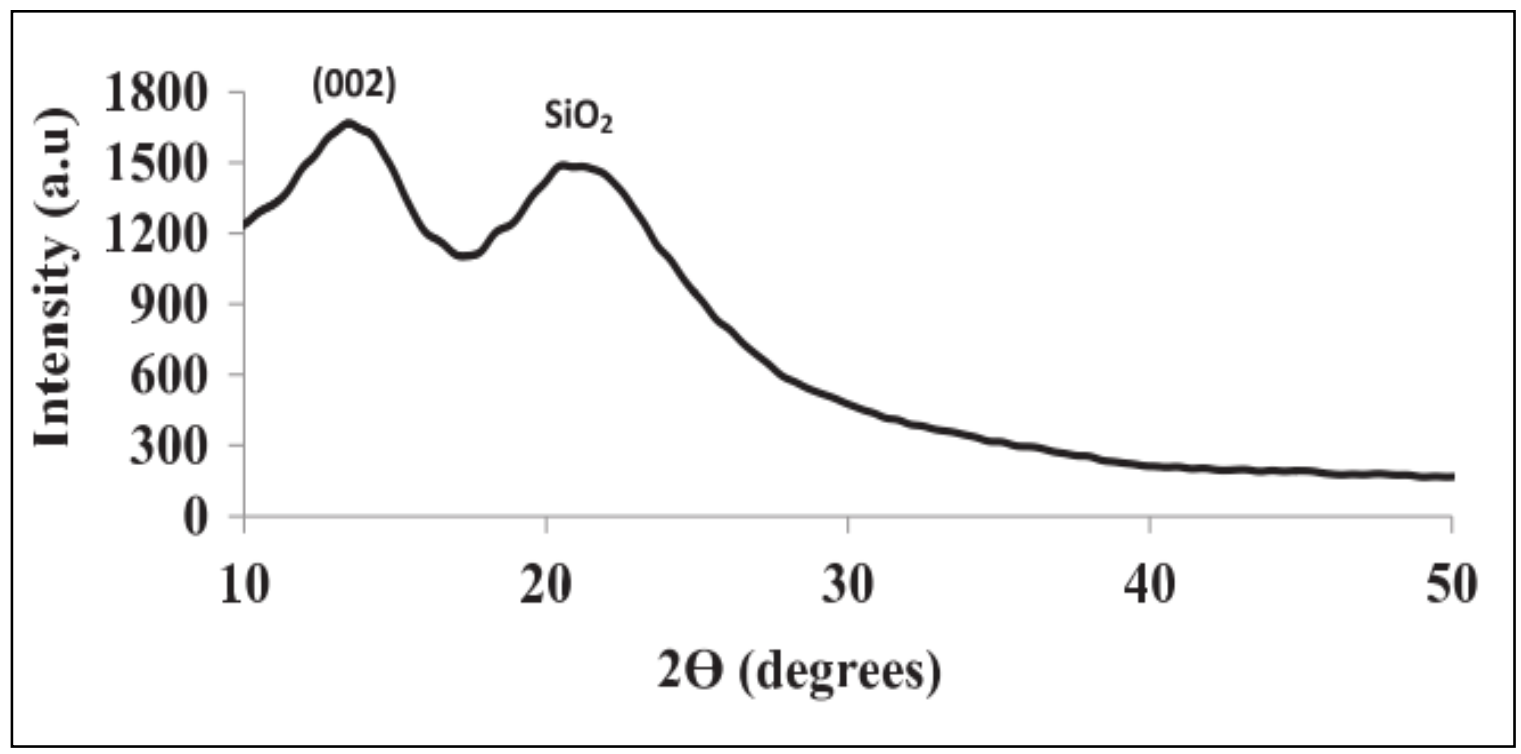

Figure 2.7: X-ray diffraction spectrum of bilayer $M o S_{2}$ thin film on a silicon oxide coated silicon substrate [28]

\subsection{ALD of Tungsten diselenide (WSe2)}

Tungsten diselenide $\left(\mathrm{WSe}_{2}\right)$ is another semiconducting layered material like $\mathrm{MoS}_{2}$, with the same type of hexagonal layer of metal atoms (tungsten) sandwiched in between two layers of chalcogenide (selenium) atoms (Fig. 2.8). $\mathrm{Like} \mathrm{MoS}_{2}, \mathrm{WSe}_{2}$ is also a direct bandgap semiconductor at the monolayer and indirect bandgap for bulk films. The indirect bandgap (for bulk) is $\sim 1.35 \mathrm{eV}$, while the direct bandgap for a monolayer is $\sim 1.65 \mathrm{eV}$. The electrical properties can vary with the metal atom sandwiched between the two layers of chalcogenide atoms. Tungsten is a larger atom than molybdenum which is 
one explanation for the difference in bandgap between $\mathrm{MoS}_{2}$ and $\mathrm{WSe}_{2}$. Another cause for variation is the chalcogen atoms size and the bonds between the metal atom and the chalcogen atoms. This variation in band gap between TMDs will be discussed later.
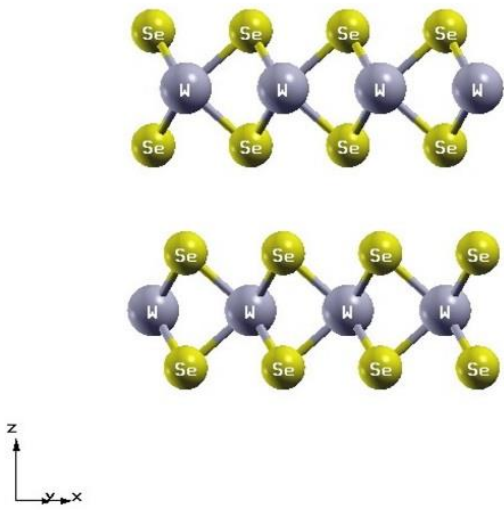
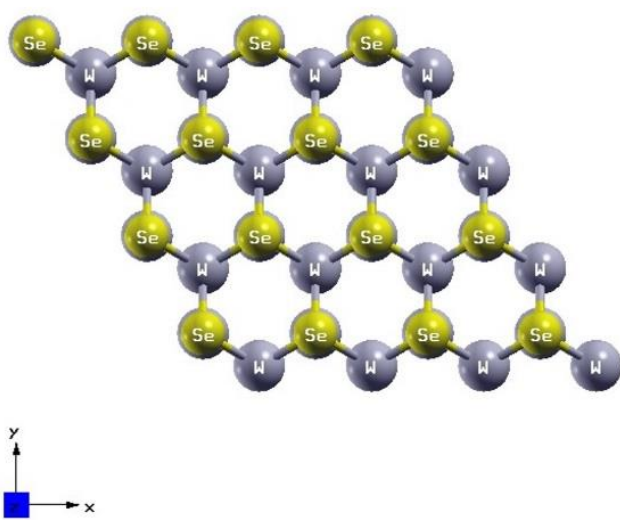

(a)

(b)

Figure 2.8: Side (a) and top (b) view of two layer $2 \mathrm{H}$ phase of $\mathrm{WSe}_{2}$

The $\mathrm{WSe}_{2}$ films were grown on silicon substrates with $31.6 \mathrm{~nm}$ thick film of silicon oxide as the dielectric. The precursors used were $\mathrm{WCl}_{6}$ and $\mathrm{H}_{2} \mathrm{Se}$ (9\% balance $\mathrm{Ar}$ ). The $\mathrm{WCl}_{6}$ source temperature was set to $110^{\circ} \mathrm{C}$ while the carrier gas (nitrogen) flow rate was $250 \mathrm{sccm}$. Growth sequence per cycle was as follows; $\mathrm{WCl}_{6}$ pulse width was $0.8 \mathrm{~s}$, $\mathrm{N}_{2}$ purge of $1.0 \mathrm{~s}, \mathrm{H}_{2} \mathrm{Se}$ pulse width of $1.2 \mathrm{~s}$, followed by $\mathrm{N}_{2}$ purge of $1.0 \mathrm{~s}$. Growth temperature was set to $390{ }^{\circ} \mathrm{C}$ and resulted in a film growth of $0.5 \mathrm{~nm} /$ cycle. Following 
the deposition, some samples were cleaved and half of the sample was annealed in Se vapor for one hour at $500{ }^{\circ} \mathrm{C}$ to improve the crystalline properties.

The annealed and unannealed films were characterized by Raman spectroscopy using an excitation wavelength of $532 \mathrm{~nm}$ and power of $1 \mathrm{~mW}$. The annealed sample showed only a minimal improvement over the unannealed half of the sample, therefore the following details are for the annealed sample. The Raman spectrum of a $3.6 \mathrm{~nm}$ thick film is shown in figure 2.9. The strongest signals occur at 247 and $256 \mathrm{~cm}^{-1}$, which are the in-plane $E_{2 g}^{l}$ and out-of-plane $A_{1 g}$ vibrational modes, respectively [38, 39]. With a monolayer film, the vibrational modes are exhibited as separate peaks, but they broaden and partially overlap as the film gets thicker. Others have shown that Raman modes are degenerate in bulk $\mathrm{WSe}_{2}$ films, showing only one peak, but this degeneracy is lifted as the film dimension is reduced [40]. The lifting of the degeneracy can possibly be explained by the addition of uniaxial strain applied to the $\mathrm{WSe}_{2}$ film from the silicon oxide layer. WSe 2 does not show the same relationship between vibrational mode peak separation and the film thickness that $\mathrm{MoS}_{2}$ does, so it is only useful to determine if the films are bulk or a few layers thick, but not how many layers. 


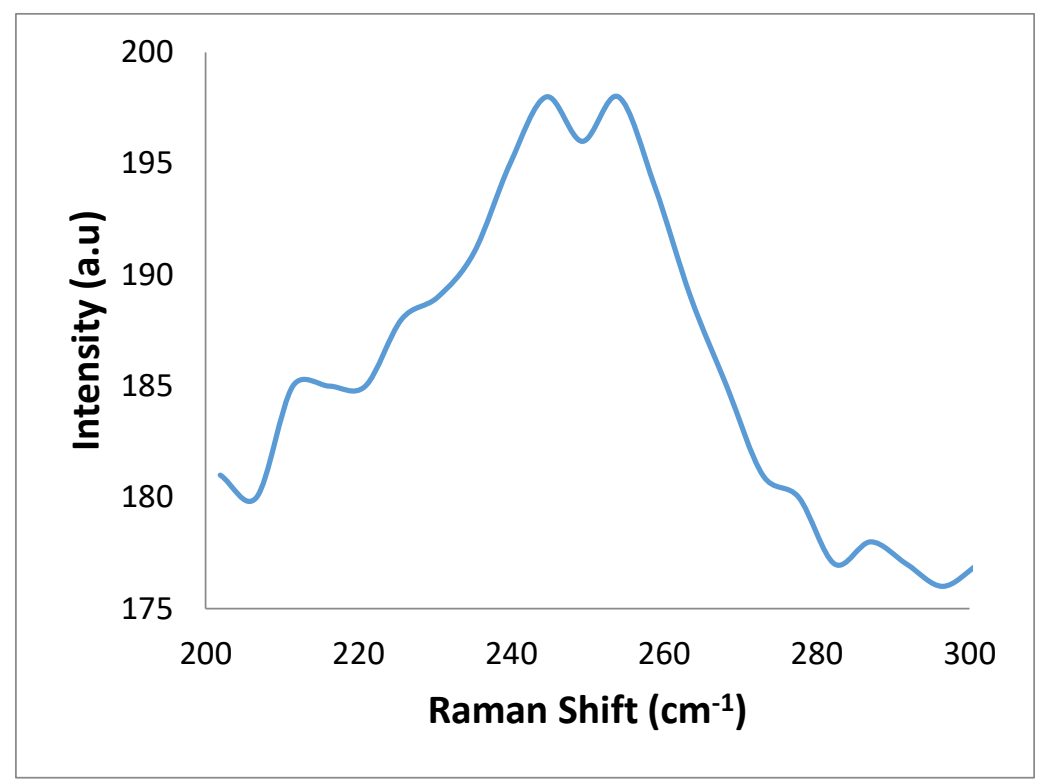

Figure 2.9: Raman spectrum of WSe 2 produced with an excitation wavelength of $532 \mathrm{~nm}$ [41].

To determine the thickness of these films, a cross-sectional profile was examined using TEM. The samples were prepared using focused ion beam (FIB) milling, which caused slight damage to the film, as can be seen in the micrograph (Fig. 2.10). A carbon film was placed on top of the $\mathrm{WSe}_{2}$ film during sample preparation. As one can see from figure 2.10, the thickness is about $0.7 \mathrm{~nm}$ for a single layer, which is consistent with the 
value reported in literature [42]. TEM analysis also showed the films to be continuous and smooth. SEM analysis did not show any discernible grain boundaries.

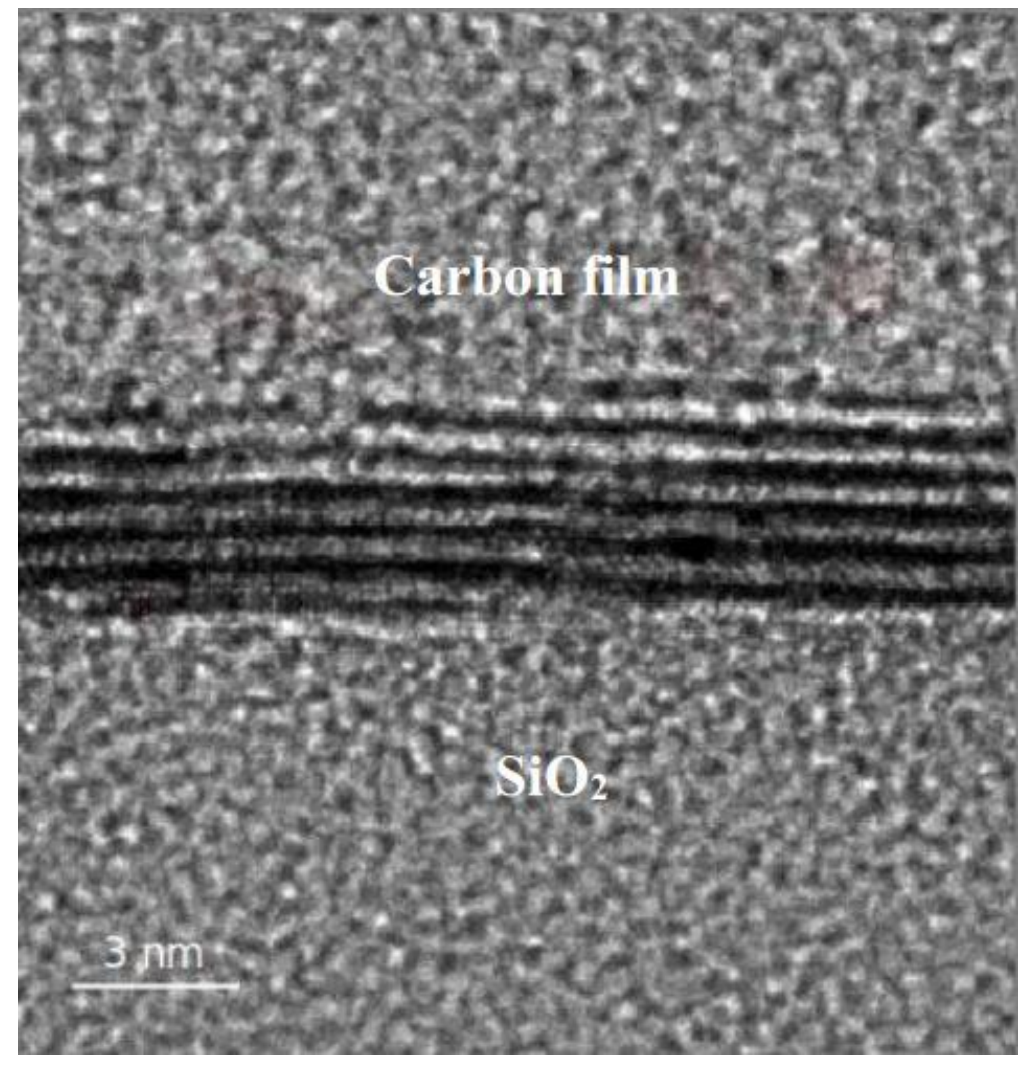

Figure 2.10: Cross-sectional TEM of a few layered thick WSe film [41]. 


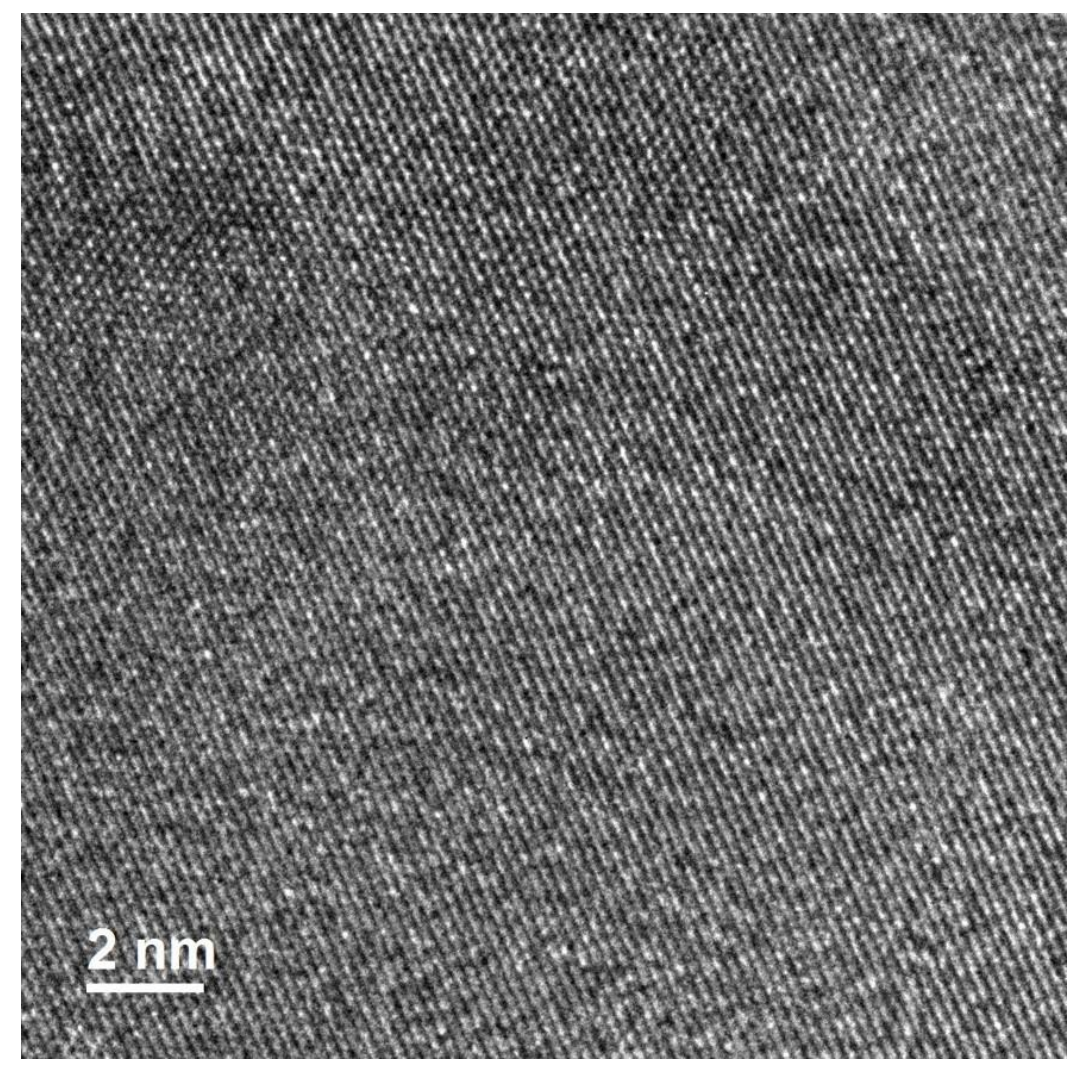

Figure 2.11: Top view TEM scan of $\mathrm{WSe}_{2}$ [41].

To examine the chemical composition and binding energies of these films, XPS was utilized. The binding energies of $\mathrm{W}$ and Se are shown in figures 2.12(a) and (b) respectively. The peaks, 32.3 and $34.6 \mathrm{eV}$ in figure 2.12 (a) are attributed to the $\mathrm{W}$ doublets $4 \mathrm{f}_{7 / 2}$ and $4 \mathrm{f}_{5 / 2}$ binding energies respectively. The tail on the $\mathrm{W} 4 \mathrm{f}_{5 / 2}$ peak is due

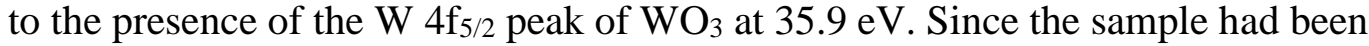
exposed to air for some time prior to XPS analysis, this most likely contributed to the extra peak. The Se binding energies are shown in figure 2.12(b). The peaks at 54.7 and 
$55.6 \mathrm{eV}$ correspond to the $\mathrm{Se} 3 \mathrm{~d}_{5 / 2}$ and $\mathrm{Se} 3 \mathrm{~d}_{3 / 2}$ binding energies. The results for both $\mathrm{W}$ and Se are consistent with XPS values reported in literature for crystalline $\mathrm{WSe}_{2}$ [43, 44].
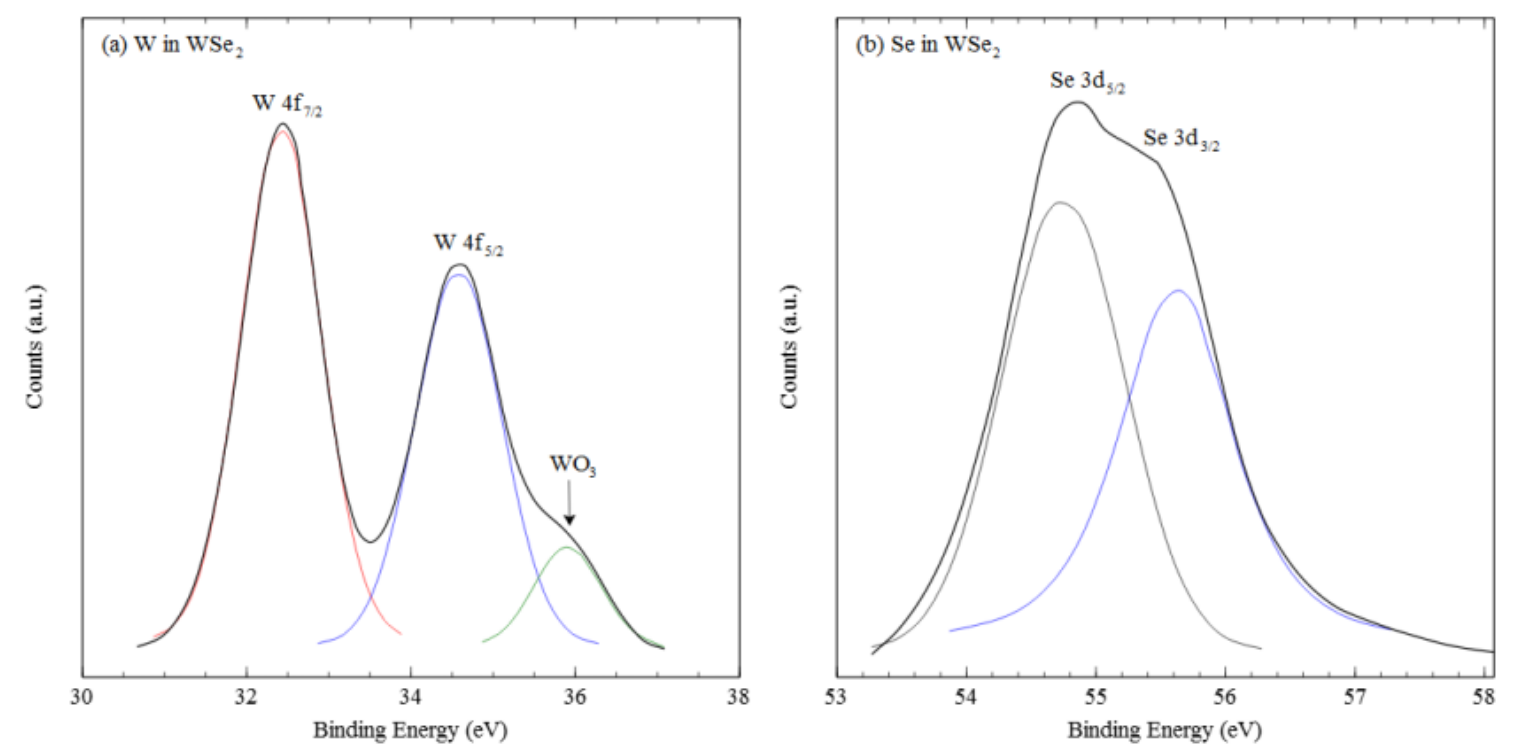

Figure 2.12: XPS spectra for binding energies of (a) W and (b) Se in ALD WSe 2 [41].

\section{5 $\mathrm{ALD}$ of $\mathrm{WS}_{2} / \mathrm{SnS}$ Heterostructure}

One of the important features of semiconducting channel materials for complimentary logic transistors, is the ability to transport electrons and holes in n-FET (field effect transistor) and p-FET devices. Historically, silicon has been doped to form nand p-type junctions, however, at this time suitable dopants are lagging for TMD materials. To address these challenges, ALD has been utilized to grow heterostructure films with both n- and p-type characteristics. Since single layers have no dangling bonds, 
the van der Waals heterostructures formed by stacking these layers should be free from lattice matching constraints and interface states, thereby leading to high performance devices.

The transition metal chalcogenide films $\mathrm{WS}_{2}$ and $\mathrm{SnS}$ were first grown in a Microchemistry F-120 ALD reactor separately, and analyzed. The reactor temperature used for both $\mathrm{WS}_{2}$ and $\mathrm{SnS}$ was $390{ }^{\circ} \mathrm{C}$. The precursors for $\mathrm{WS}_{2}$ were $\mathrm{WCl}_{6}$ and $\mathrm{H}_{2} \mathrm{~S}(9 \%$ Ar balance). The $\mathrm{WCl}_{6}$ source temperature was $110{ }^{\circ} \mathrm{C}$ and the carrier gas (nitrogen) flow rate was $250 \mathrm{sccm}$. The pulse sequence per cycle was as follows; $\mathrm{WCl}_{6}$ pulse width was $0.8 \mathrm{~s}, \mathrm{~N}_{2}$ purge for $1.0 \mathrm{~s}, \mathrm{H}_{2} \mathrm{~S}$ pulse of $1.2 \mathrm{~s}$, followed by $1.0 \mathrm{~s} \mathrm{~N}_{2}$ purge. The film growth rate was $0.125 \mathrm{~nm}$ per cycle. This film thickness, measured with AFM, was $2.5 \mathrm{~nm}$ and was analyzed with Raman spectroscopy using $532 \mathrm{~nm}$ wavelength laser excitation at a power of $1 \mathrm{~mW}$. The Raman spectrum shown in figure 2.13 is similar to what has been reported in literature for the $2 \mathrm{H}$ stacked phase [45]. The first order optical modes $\mathrm{E}^{1}{ }_{2 \mathrm{~g}}$ occurs at $355 \mathrm{~cm}^{-1}$ and $\mathrm{A}_{1 \mathrm{~g}}$ occurs at $418 \mathrm{~cm}^{-1}$ while second order longitudinal acoustic mode $2 \mathrm{LA}(\mathrm{M})$ at the $\mathrm{M}$ point, occurs at $353 \mathrm{~cm}^{-1}$. Since the $\mathrm{E}^{1}{ }_{2 \mathrm{~g}}$ and $2 \mathrm{LA}(\mathrm{M})$ modes are so close together, they overlap [46]. There is another peak around $300 \mathrm{~cm}^{-1}$ that is due to another 2LA(M) [46]. 


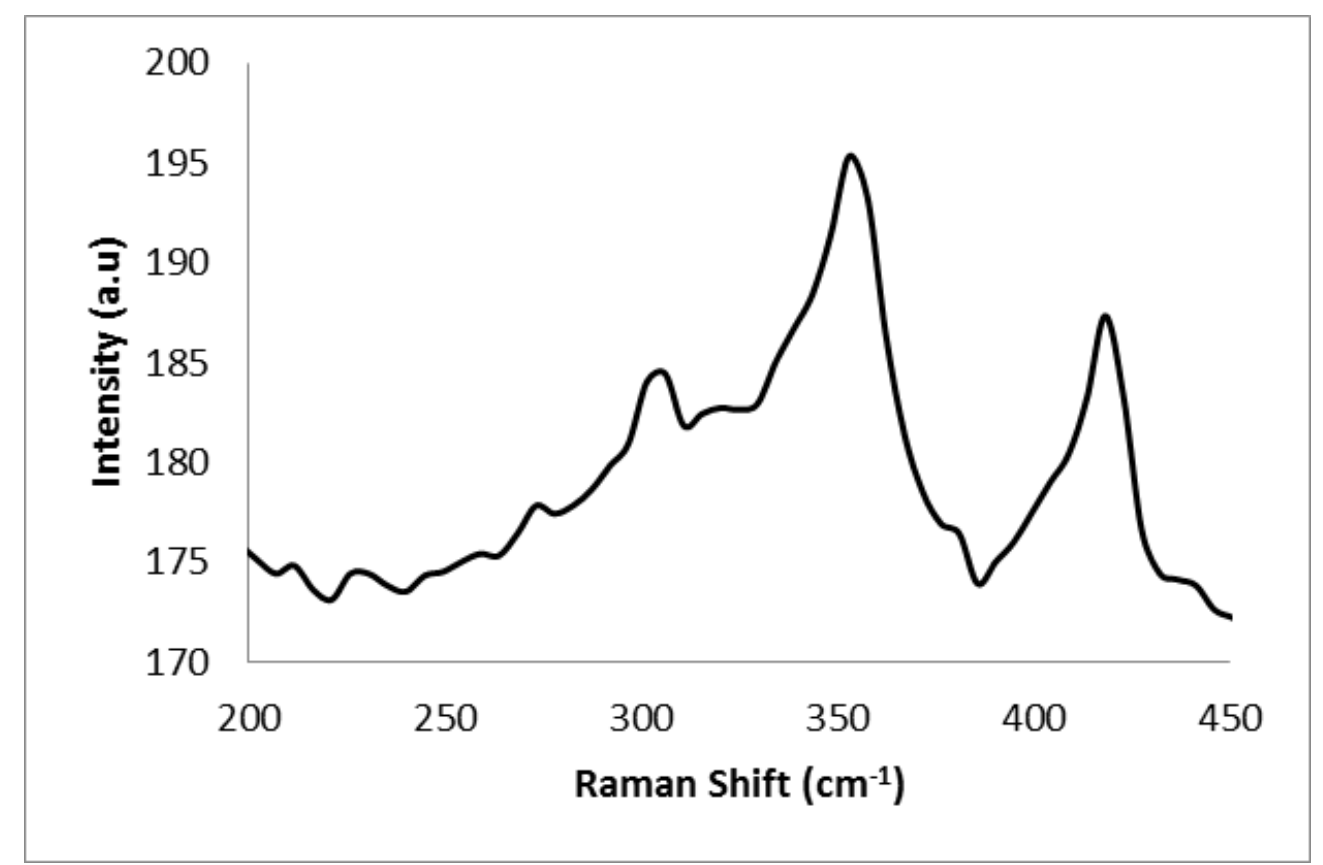

Figure 2.13: Raman spectrum of $\mathrm{WS}_{2}$ film [47].

The $\mathrm{SnS}$ films were grown from tin(II) acetylacetonate and $\mathrm{H}_{2} \mathrm{~S}$. The tin source temperature was $95{ }^{\circ} \mathrm{C}$ and the pulse sequence was as follows; tin $0.6 \mathrm{~s}, \mathrm{~N}_{2}$ purge for 1.0 $\mathrm{s}, \mathrm{H}_{2} \mathrm{~S}$ pulse of $1.2 \mathrm{~s}$, and $\mathrm{N}_{2}$ purge of $1.0 \mathrm{~s}$. The growth rate was $0.47 \mathrm{~nm}$ per cycle. At lower growth temperatures $\left(<250{ }^{\circ} \mathrm{C}\right)$ the films were composed of mixed phases, however above $300{ }^{\circ} \mathrm{C}$ only $\mathrm{SnS}$ phase films were produced, as verified by Raman spectroscopy. The Raman spectrum from a $4.5 \mathrm{~nm}$ thick film (measured by AFM) is shown in figure 2.14, where the laser wavelength was $532 \mathrm{~nm}$. Several peaks were observed, at 95, 162, 190, 225, and $287 \mathrm{~cm}^{-1}$, and are consistent with those reported for single crystal $\mathrm{SnS}$ at $94,162,191,219$, and $288 \mathrm{~cm}^{-1}[46,48]$. The origin of these peaks 
has been attributed to first order single phonon oriented transverse or longitudinal optical vibrational modes [49].

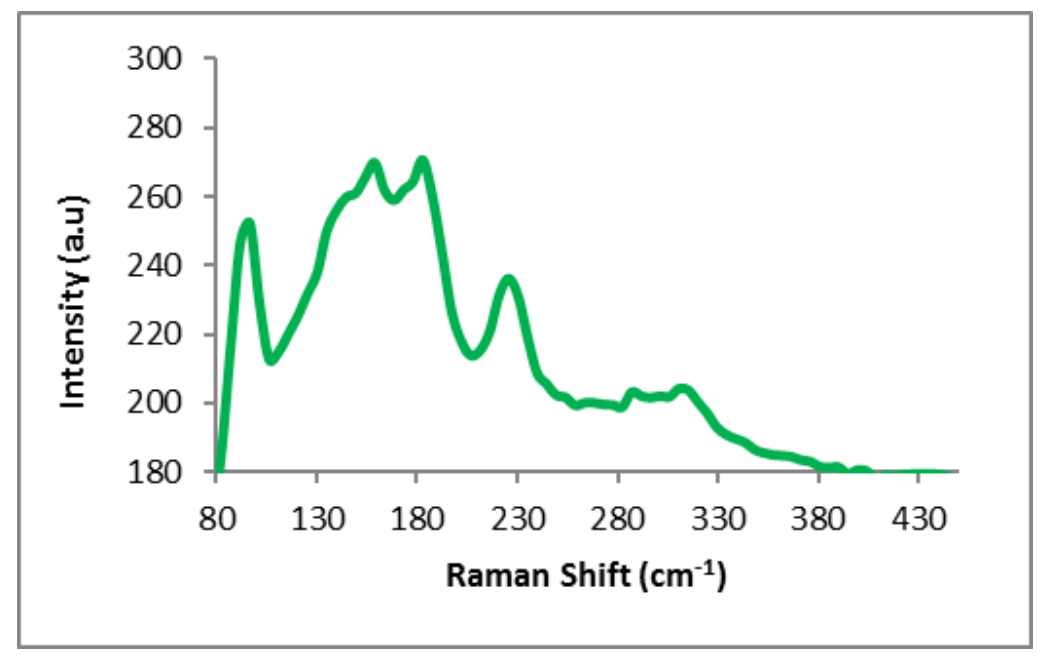

Figure 2.14: Raman spectrum of SnS film [47]

After growing individual $\mathrm{WS}_{2}$ and $\mathrm{SnS}$ films and optimizing growth parameters, heterostructures were then created by alternately growing $\mathrm{WS}_{2}$ and $\mathrm{SnS}$ layers using the same parameters as the individual films growth. The thickness of each layer was controlled by selecting the appropriate number of cycles of the films respective precursors. The thickness of each material was varied from a couple of layers to 10 layers. When viewed under an SEM, the surfaces of these films were smooth and continuous and there were no visible grain boundaries. Raman spectroscopy was performed on one of the heterostructure samples and is shown in figure 2.15. It shows the 
two optical modes of $\mathrm{WS}_{2}$ but not the $2 \mathrm{LA}(\mathrm{M})$ at $300 \mathrm{~cm}^{-1}$ that was seen for the individual film. Both peaks are shifted towards the higher frequency by $2 \mathrm{~cm}^{-1}$.

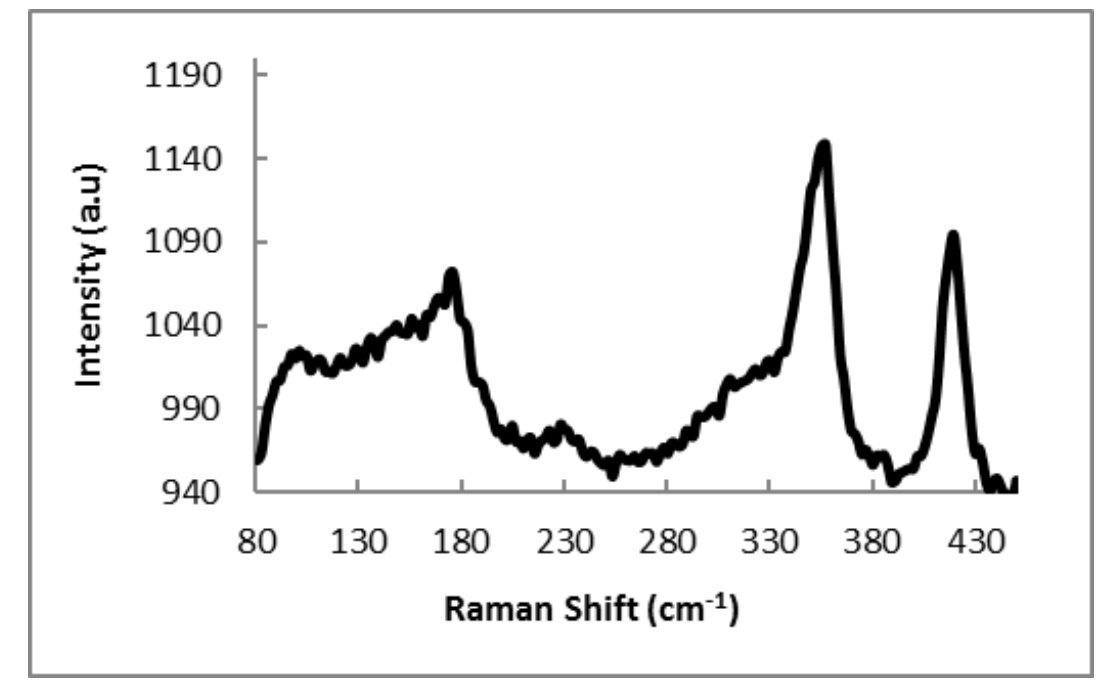

Figure 2.15: Raman spectrum of the $W S_{2} / S n S$ heterostructure [47]

After the deposition of the films, a small section of the sample was cleaved for observation with a TEM. The TEM samples were prepared using FIB milling, which proved to be very difficult for the very thin films, especially the $\mathrm{SnS}$ layers, since the FIB process damaged the films. A cross-sectional TEM view of a film composed of three pairs of $\mathrm{WS}_{2} / \mathrm{SnS}$ layers is shown in figure 2.16. This layer had the thickest $\mathrm{SnS}$ layer and therefore suffered the least amount of FIB damage, however the damage at the interface is evident. This low magnification TEM is shown to demonstrate the continuous nature of the film. The darker layers are $\mathrm{WS}_{2}$ and the lighter layers are SnS. A close-up of one of the $\mathrm{WS}_{2} / \mathrm{SnS}$ interfaces from an undamaged portion of the film stack is shown in figure 
2.17. A continuous transition can be observed from $\mathrm{WS}_{2}$ to $\mathrm{SnS}$ layers. The four layers of $\mathrm{WS}_{2}$ and the SnS lattice are clearly visible.

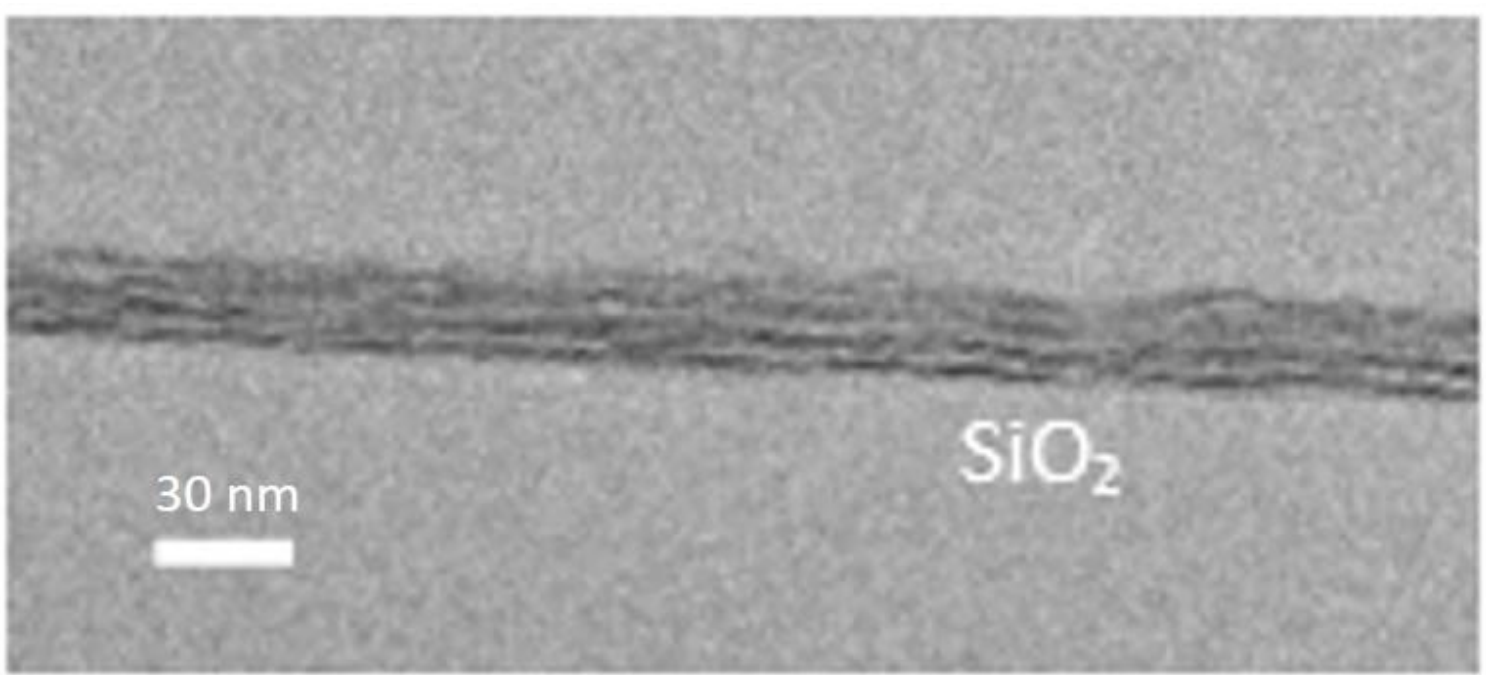

Figure 2.16: Cross-sectional TEM of 3 alternating sets of $W S_{2} / S n S$ layers, starting with $W S_{2}$ at the bottom [47]. 


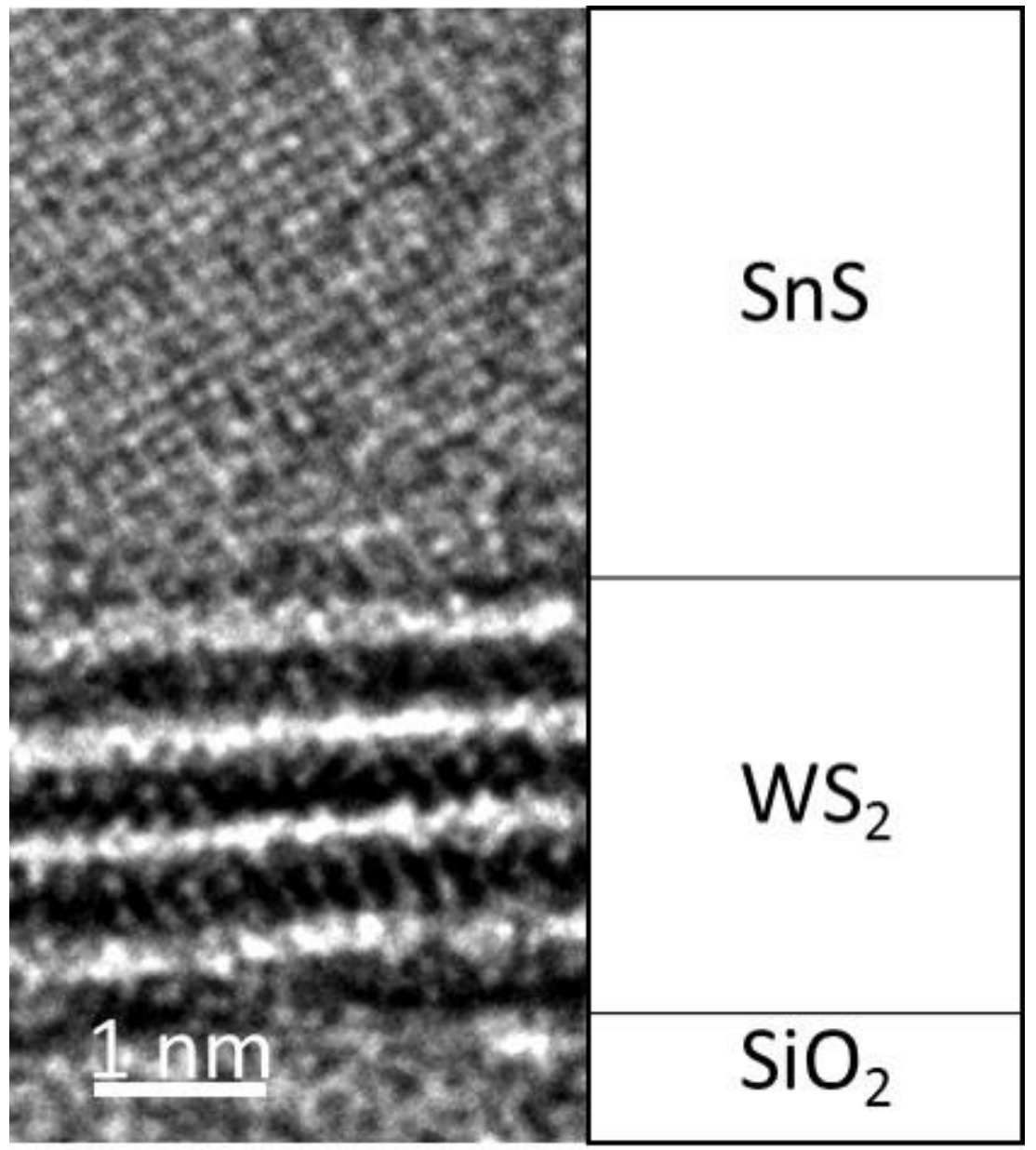

Figure 2.17: Cross-sectional TEM micrograph of the $W_{2} / S n S$ interface [47].

The $\mathrm{WS}_{2}$ film displays a clear lattice only in the axial direction, where the spacing between layers is measured to be about $6.2 \AA$. A comparison between this value and unstrained $\mathrm{WS}_{2}(\mathrm{c}=6.145 \AA$ ) [JCPDS 08-0237] confirms that the film is mono-phased and hexagonal. In the SnS portion of the film, the peaks visible in the Fast Fourier Transform (FFT) analysis in figure 2.18 correspond to the $\{0,1,1\}$ family of reflections, 
and therefore the orientation corresponds to the [100] zone axis. The $\{0,0,1\}$ reflections are not present due to the reflection condition of the Pnma group. The lattice parameters were determined to $c=4.06 \AA$, and $a=4.36 \AA$, which matches with the Herzenbergite SnS (JCPDS 39-0354, $b=3.9838 \AA$, $c=4.3291 \AA$ ). There is a stretch of about $2.2 \%$ in the $b$-direction and $0.6 \%$ in the $c$-direction.

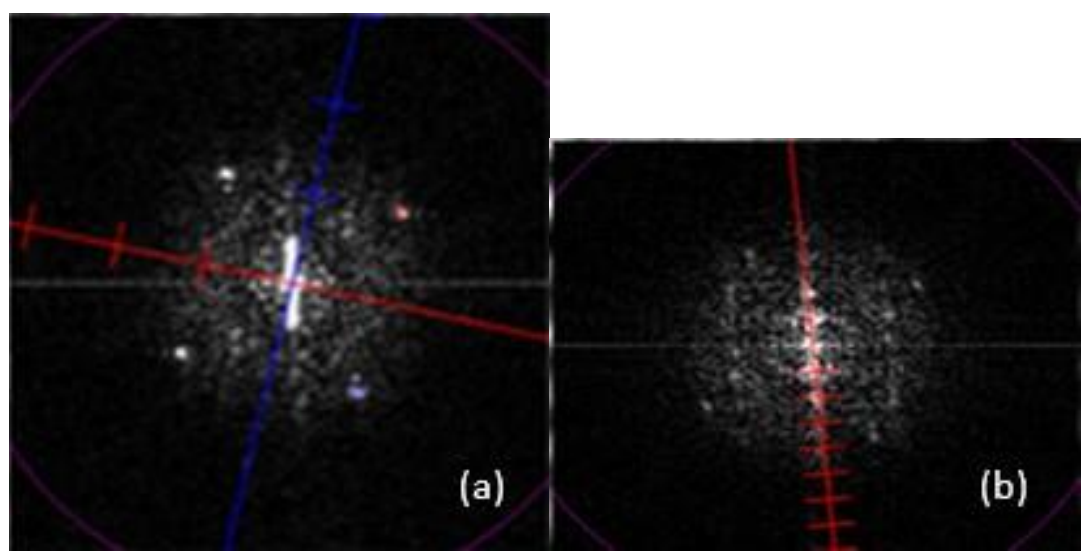

Figure 2.18: (a) FFT of SnS part of the film stack. Lattices are shown on the FFT. Blue is the $c^{*}$ axis of $S n S$ and red is the $b^{*}$ lattice of $S n S$. (b) FFT of the $W S_{2}$ part of the film stack [47].

A very interesting finding was that although both $\mathrm{WS}_{2}$ and $\mathrm{SnS}$ are layered materials, the FFT analysis of the high resolution TEM (HRTEM) images reveals that the orientation of the $\mathrm{SnS}$ layers is not parallel to the $\mathrm{WS}_{2}$ layers. To reveal the mutual orientation of the two lattices, an FFT from the interface area was performed. As can be seen from figure 2.19 , the angle between $\mathrm{c}^{*}\left(\mathrm{WS}_{2}\right.$, green) and $\mathrm{c}^{*}(\mathrm{SnS}, \mathrm{blue})$ is about 15 degrees. The closest low index zone axis which corresponds to this tilt, is the [410] axis, 
with angle to [001] being $14.03^{\circ}$, thus the orientation relation between the two components of the heterostructure is [410]SnS/[001] $\mathrm{WS}_{2}$. This angular orientation continues at other interfaces in the stack. This is counterintuitive, since one would expect that two layered materials will pack in a way that preserves their layered structure within the heterostructure, as in the case of $\mathrm{MoS}_{2} / \mathrm{WS}_{2}$ heterojunctions, where stacked layers of $\mathrm{MoS}_{2}$ and $\mathrm{WS}_{2}$ preserved the $2 \mathrm{H}$ stacking [50]. In this case both the layers had similar $2 \mathrm{H}$ phase crystal structures [50]. However, in our case the two layered materials have different crystal structures where the layered nature of the materials is preserved, however the growth occurs at an angle with respect to their van der Waals planes. This misaligned growth of the two films will have implications on the electrical properties of the heterostructure which will be discussed later.

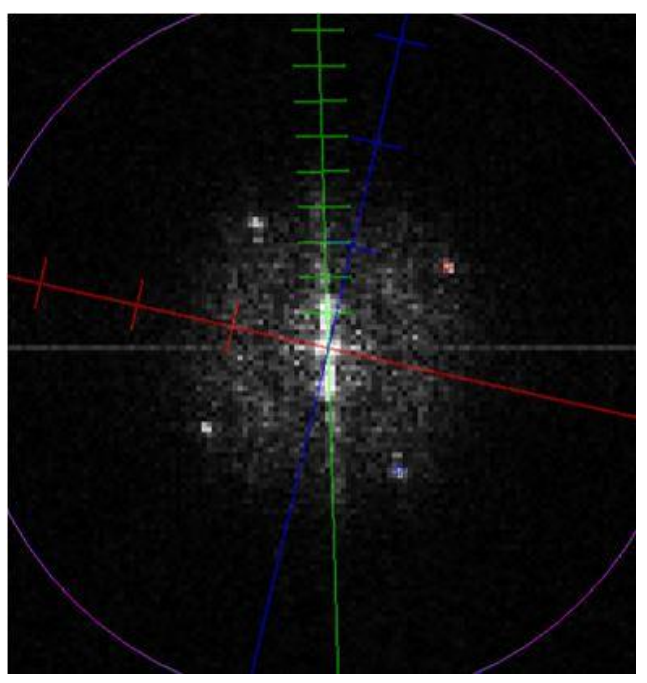

Figure 2.19: FFT of the interface section of Fig. 2.17 [47]. The green lattice corresponds to the $W_{2}$ interlayer spacing. The blue $c^{*}$ axis of $S n S$, red $b^{*}$ axis of $S n S$. 


\subsection{Summary}

The growth of several TMD films using ALD has been demonstrated. Continuous uniform films of $\mathrm{MoS}_{2}, \mathrm{WSe}_{2}, \mathrm{WS}_{2}, \mathrm{SnS}$, and $\mathrm{WS}_{2} / \mathrm{SnS}$ heterostructures were grown over a $5 \times 5 \mathrm{~cm}$ area. An important note is that this is the first time uniform 2D TMD films have been produced over such a large area. During the growth of the TMD films, several analytical techniques were employed to study their physical characteristics and to ensure a comprehensive examination of the film. For example, the Raman spectrum for $\mathrm{MoS}_{2}$ shows a clear correlation between the thickness of the film and the separation of the inplane and out-of-plane vibrational modes, which matches well with literature. $\mathrm{WSe}_{2}$ does not have a similar thickness correlation, however when film growth is limited to only a few layers thick, the individual peaks in the in-plane and out-of-plane vibrational modes start to become evident. In the case of the $\mathrm{WS}_{2} / \mathrm{SnS}$ heterostructure, the Raman peaks were a combination of the individual films with some minor shift for $\mathrm{WS}_{2}$. Upon further analysis, XPS of both $\mathrm{MoS}_{2}$ and $\mathrm{WSe}_{2}$ clearly indicated the chemical composition of those two films match that of crystalline films which was also confirmed by XRD for $\mathrm{MoS}_{2}$. Photoluminescence examination of $\mathrm{MoS}_{2}$ was able to show that a bi-layer film produced surprisingly strong photoluminescence. No film thicknesses fewer than 3-4 layers were grown for $\mathrm{WSe}_{2}$, therefore no photoluminescence was performed for that film. The analyses performed with SEM, TEM, and AFM further demonstrate that these films are good quality ALD grown films. 
The monolayer stepwise growth process that ALD uses, makes this method of film growth advantageous over other methods of growing or depositing few layers films. The ability to grow TMD films a few monolayers thick is particularly important for semiconductor device manufacturing. Most research to date has come from examining small samples (on the order of a few tens of microns) of exfoliated films because the weak van der Waals force makes this an easy method from which to obtain everything from bulk to single layer films. This research has shown that ALD can grow good quality uniform films over large areas with similar properties to the exfoliated films, making ALD an attractive process for growing these materials. 


\section{Chapter III: Electrical Characterization}

The study of graphene, with its exceptional electrical qualities, has sparked great interest in the TMDs, with the hope that they also possess some of the electrical properties that graphene possesses. The electrical characterizations performed on semiconducting TMD thin film layers created from exfoliated flakes have shown some interesting electrical properties, which begs the question, can these same properties be seen on ALD grown thin films? In contrast to graphene's zero bandgap, the semiconducting TMDs have a bandgap of between 1-2 eV, which makes them good candidates for electronic devices requiring high $\mathrm{I}_{\mathrm{on}} / \mathrm{I}_{\mathrm{off}}$ ratios. TMDs such as $\mathrm{MoS}_{2}$ and $\mathrm{WSe}_{2}$ also exhibit a direct bandgap with strong photoluminescence when the films are a monolayer thick, making them good candidates for optoelectronics. Other important electronic properties the TMDs have exhibited are large $I_{o n} / I_{o f f}$ ratios [51], ultra-low offstate energy dissipation (i.e. low leakage), high field effect mobility [51], large current carrying capacity $[17,52,53]$, and a high degree of immunity from short channel effects [53]. To understand if the same electronic properties are exhibited with ALD grown films, wafers with several TMD films grown via ALD were patterned using optical lithography, and back-gated transistors were built and electrical characterization was performed.

\subsection{Device Fabrication and Measurement Techniques}

\subsubsection{Photolithography}


To create back-gated FETs, silicon wafers were prepared with thermally grown silicon oxide at varying thickness (30 to $320 \mathrm{~nm}$ ) and ALD grown TMD films as previously described. These wafers were patterned to open the area for the source and drain contacts where the metal was deposited. The photoresist used in patterning the contacts was Shipley Microposit S1813 positive photoresist. The resist was spun on the wafer with a spin coater at 3200 RPM for 110 seconds, completely coating the wafer with a resist film thickness of approximately $1.5 \mu \mathrm{m}$. After the spin coating was performed, the wafer underwent a soft bake process at $100^{\circ} \mathrm{C}$ on a hotplate for 60 seconds to improve resist adhesion and prevent sticking to the contact mask. The wafers were then exposed to $365 \mathrm{~nm}$ wavelength UV light for 120 seconds using a OAI Hybralign 200 Contact Aligner (Fig. 3.1). 


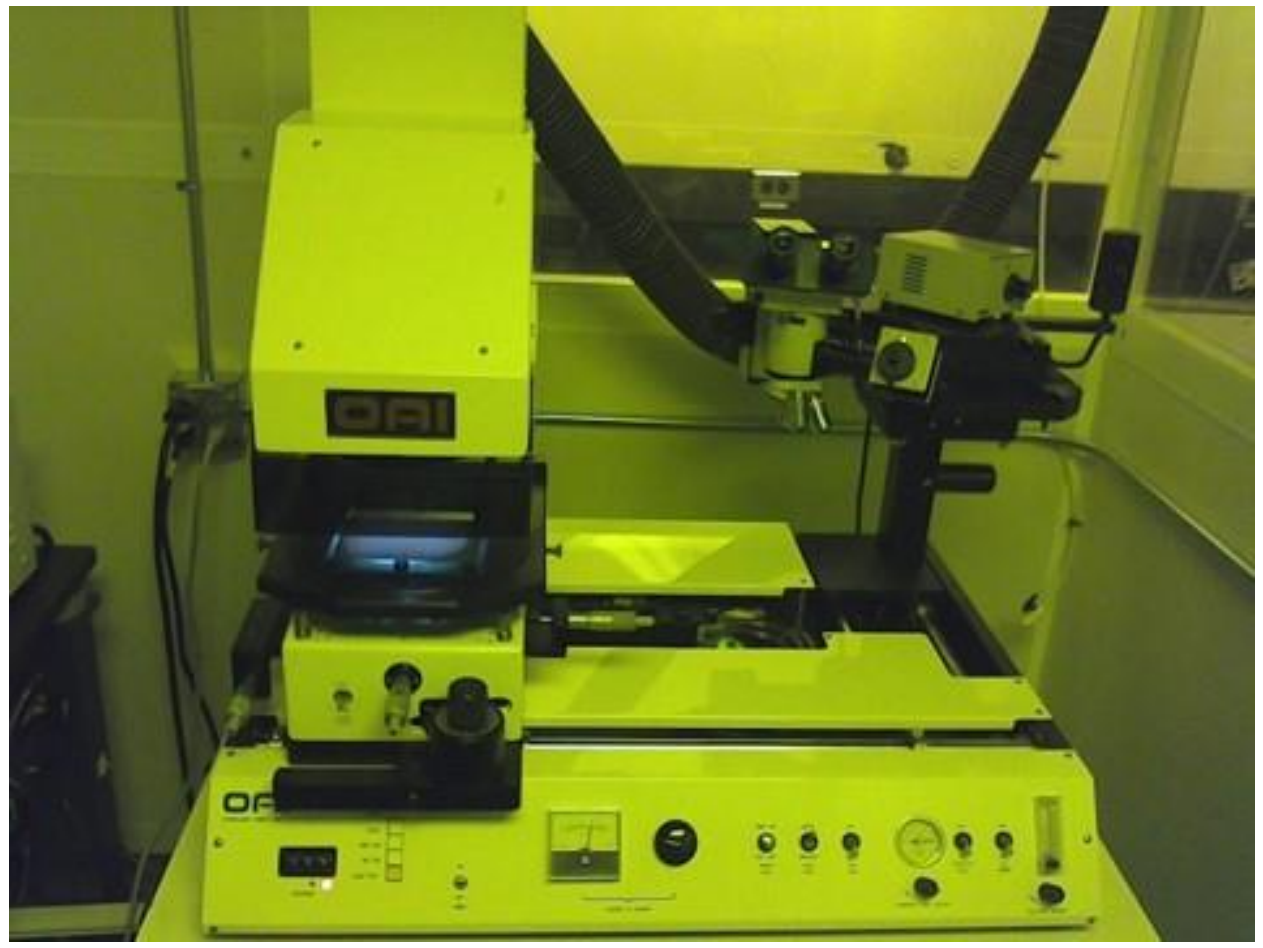

Figure 3.1: OAI Hybralign 200 Contact Aligner

The contact mask was a 5-inch soda lime glass mask with $2 \mu \mathrm{m}$ resolution and a dense population of features to create transistors with gate lengths ranging from 2 to 10 $\mu \mathrm{m}$ and $100 \mu \mathrm{m}$ channel width. A set of transmission line method (TLM) structures were also created to enable performing contact and sheet resistance measurements. After the wafers were exposed, they were developed in a $\mathrm{NaOH}$ solution between 0.1325 and 0.194 $\mathrm{M}$ concentration to remove the exposed photoresist, thereby opening the areas for metal deposition to create the transistor contacts. It was necessary to vary the $\mathrm{NaOH}$ solution concentration to create the proper develop time, taking into account photoresist age and thickness so that good pattern uniformity could be achieved across the wafer. Developing 
too fast caused poor uniformity, creating overdeveloped features in the center and underdeveloped features at the edge of the wafer. Total develop time used for all films was between 15 and 20 seconds. After patterning, metal deposition was performed using various metals ( $\mathrm{Ti}, \mathrm{Au}, \mathrm{Pd}$, and $\mathrm{Ni}$ ) to compare contact properties with the different films. Metal was deposited between 30 and $60 \mathrm{~nm}$ thick (very early samples had 80nm thick Ti) using electron beam and sputter deposition processes. After the metal deposition process, the excess metal and unexposed photoresist were lifted off the wafer using acetone. The fabrication process was then completed by applying Ag paste to the wafer backside, to provide the backside gate contact. An optical microscope view of one of the areas of a patterned wafer with metal contacts is shown in figure 3.2. The numbers to the right of the features were part of the mask pattern and indicate the channel length in micrometers, so that we knew the gate length when making the electrical calculations after measurements were made. Each cluster consists of five transistors with gate lengths of 2, $4,6,8$, and $10 \mu \mathrm{m}$ and two sets of TLM structures, each of widths (distance between the metal contacts) of $20,40,60,80$, and $100 \mu \mathrm{m}$ and length of $500 \mu \mathrm{m}$. Figure 3.3 is a sample of one of the wafers showing the densely packed set of features. 


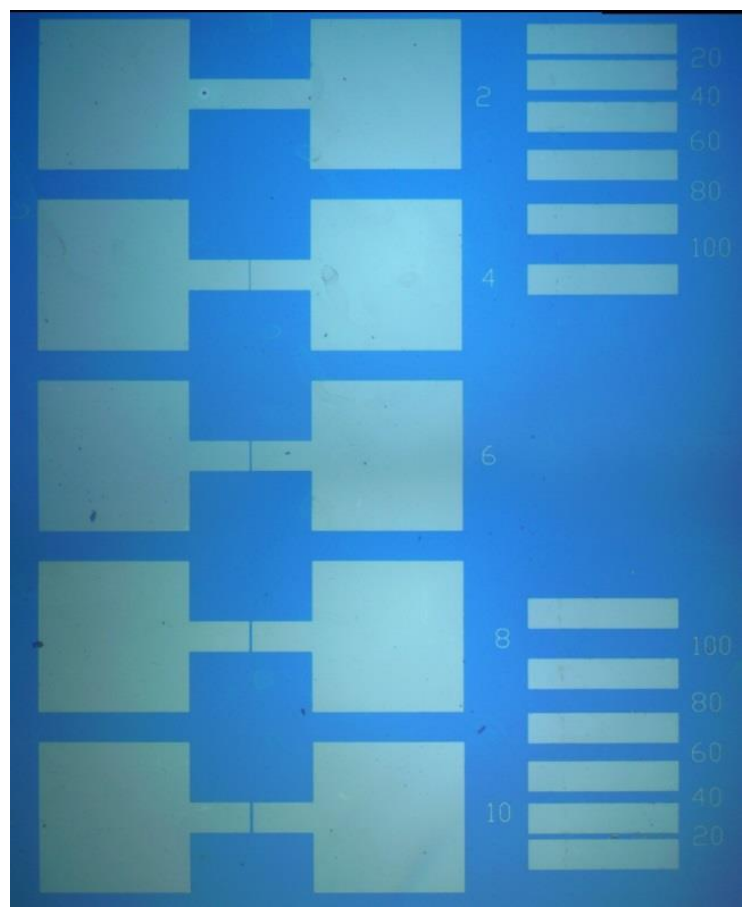

Figure 3.2: Close up view of completed wafer, patterned with metal contacts showing the individual transistors and TLM structures. 


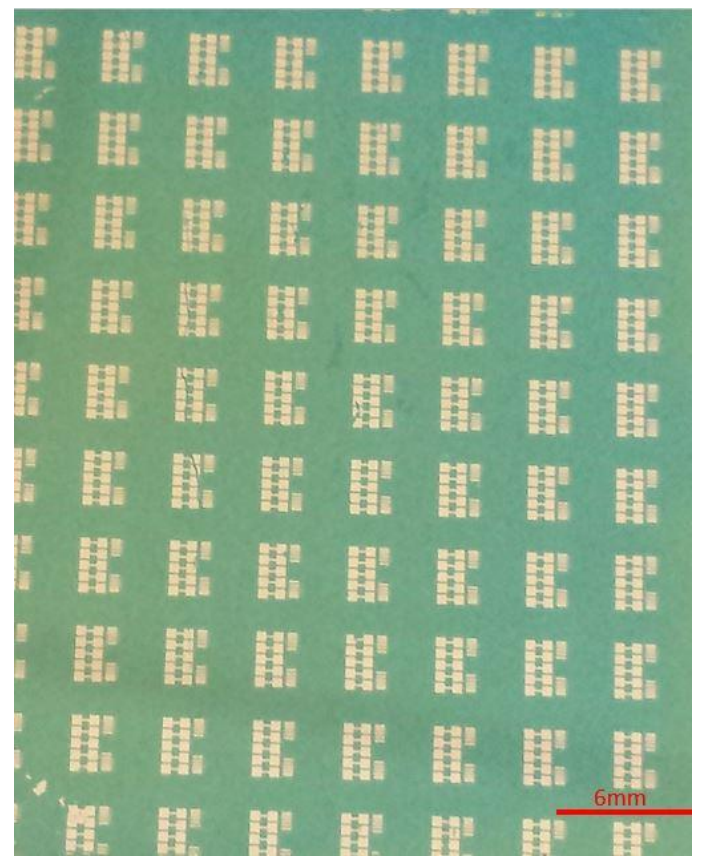

Figure 3.3: WSe2 wafer patterned with metal deposition.

\subsubsection{Electrical Equipment and Tests}

Electrical characterization is an important component to understanding the nature and operation of the semiconducting TMD films. The electrical tests performed included standard $I_{d}-V_{d}$ and drain current versus gate voltage tests using back-gated transistors, which provided conductance, mobility, and current on/off ratios. The TLM structures enabled the performance of sheet and contact resistance measurements which provided valuable information about the film resistance and contact characteristics of the different metals used. 
The electrical tests were performed using a probe station (Fig. 3.4) connected to an HP 4145B Semiconductor Parameter Analyzer (Fig. 3.5). The output of the analyzer is input to a computer running Metrics Technology ICS (Interactive Characterization Software) which controls the input/output parameters for each test and stores and plots data. The probe station has an enclosure to allow samples to be tested in a dark environment which is beneficial for films that are sensitive to light.

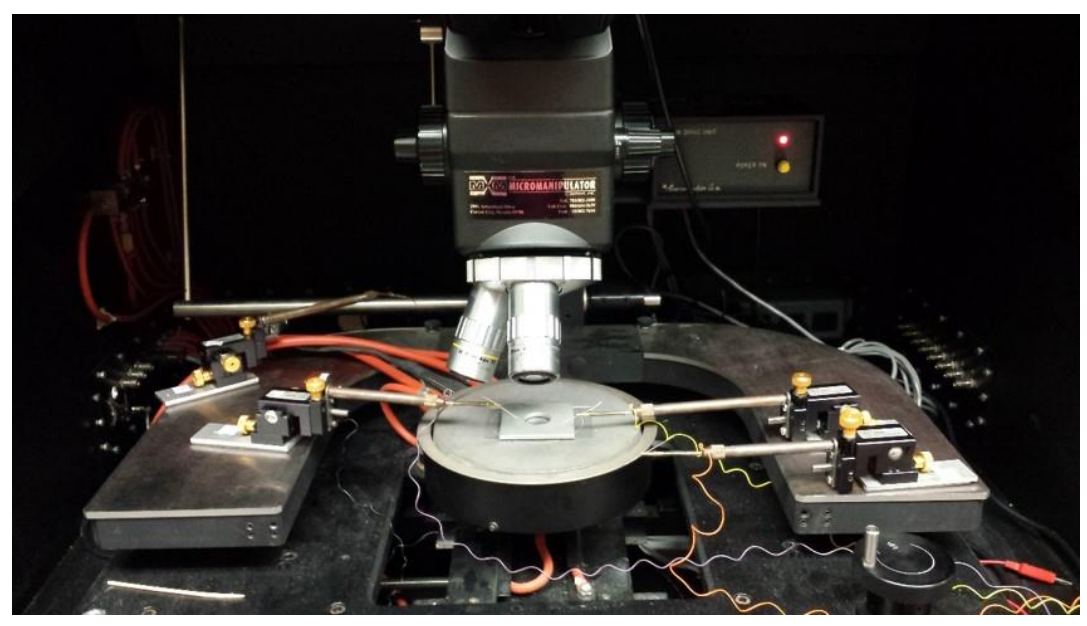

Figure 3.4: Probe Station 


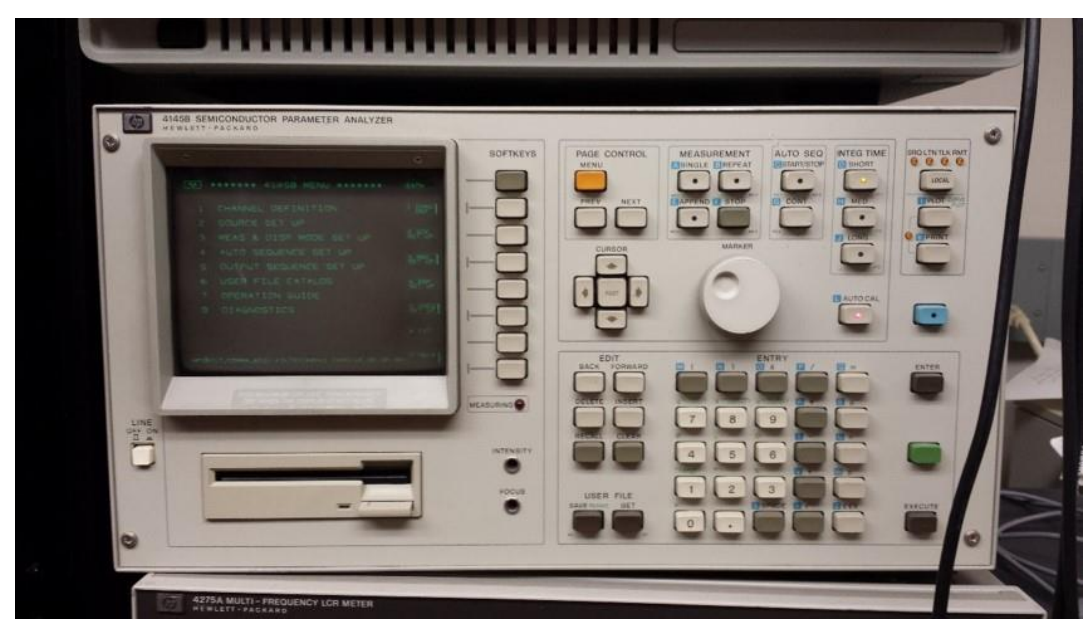

Figure 3.5: HP 4145B Semiconductor Parameter Analyzer

\section{2 $\mathrm{MoS}_{2}$ Electrical Data}

The electrical properties of the bilayer film of $\mathrm{MoS}_{2}$ (described in section 2.3) were studied using back-gated FETs like those in figure 3.2. The film was grown on top of $\sim 320 \mathrm{~nm}$ silicon oxide which acted as the gate oxide. The metal contacts were made from $80 \mathrm{~nm}$ thick Ti, deposited via electron beam deposition. Choosing low resistance metal contacts are crucial because high resistance metal contacts limit TMD device performance. Ti was selected since its work function $(\sim 4.3 \mathrm{eV})$ is closer to the conduction band $(\sim 4.7 \mathrm{eV})$ of $\mathrm{MoS}_{2}$, anticipating an n-type behavior in the film, thereby creating a smaller Schottky barrier. The Schottky barrier $\left(\Phi_{\mathrm{B}}\right)$ is a potential energy barrier that is formed at a metal-semiconductor junction. The barrier between the metal and semiconductor is predicted by the Schottky-Mott rule $\left(\Phi_{B}=\Phi_{M}-\chi\right)$, where $\Phi_{M}$ is the metal work function and $\chi$ is the $\mathrm{MoS}_{2}$ electron affinity, to be proportional to the difference between the metal-vacuum work function and the electron affinity of the 
semiconductor. In practice, the chemical termination of the semiconductor crystal against the metal creates electronic states within its bandgap. These metal induced states and their occupation by electrons tends to pin the center of the bandgap to the Fermi level, known as Fermi level pinning. This can cause the height of the Schottky barrier to vary from the predicted value of the semiconductor or metal work function. Ti has a low metal work function, favorable interface geometry and bonding, a large density of states at the Fermi level, and a narrow potential barrier at the interface region to $\mathrm{MoS}_{2}$, creating a good contact metal for electron injection $[54,55]$. Initially, the gate bias was set to zero while sweeping the drain voltage and measuring the drain current. The $I_{d^{-}} V_{d}$ profile was linear (Fig. 3.6), indicating Ohmic contacts, although some of the earlier $\mathrm{MoS}_{2}$ samples did show slight Schottky contacts. The reason for the Schottky contact is unknown, but it is possible the interface between the metal and $\mathrm{MoS}_{2}$ layer was poor, leading to a larger Schottky barrier height. 


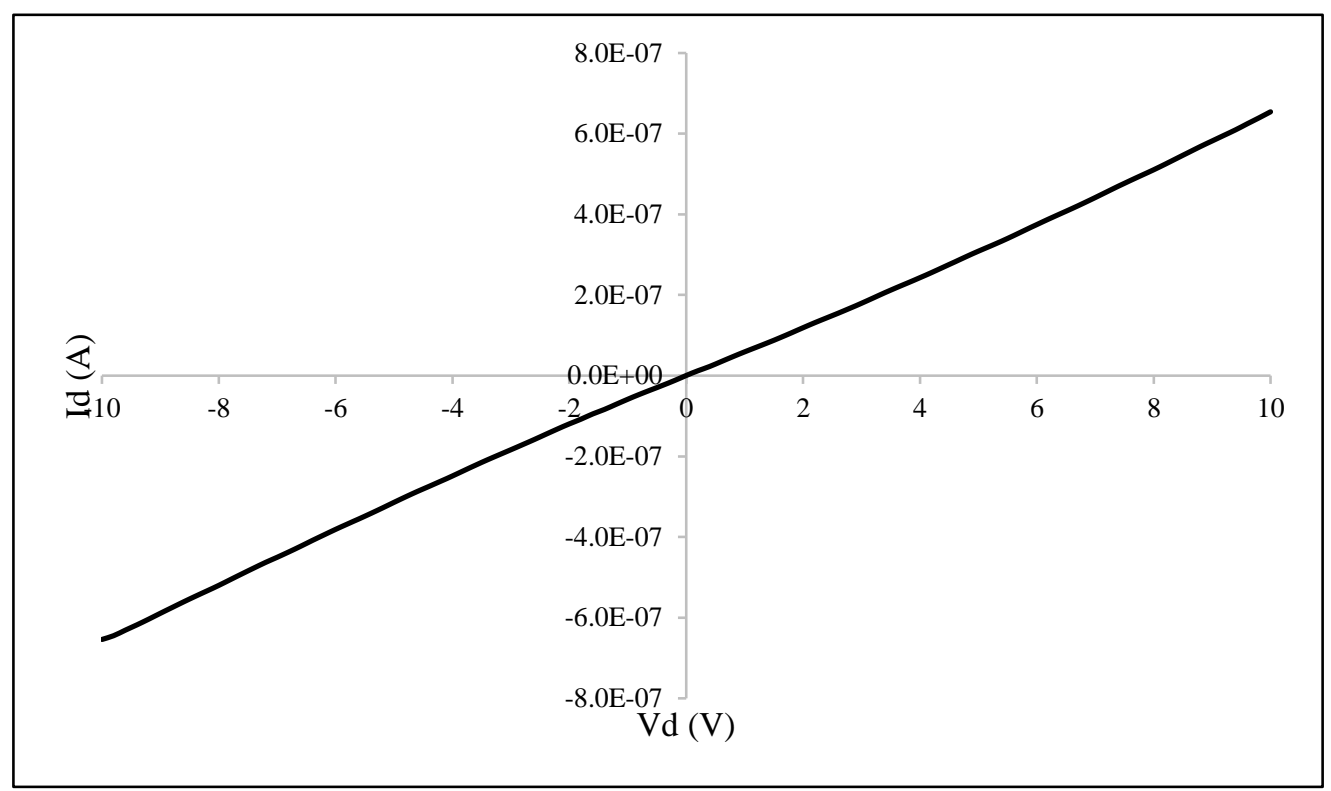

Figure 3.6: Drain current $\left(I_{d}\right)$ vs. drain voltage $\left(V_{d}\right)$ of $M o S_{2}$ bilayer film

Next, the dependence of drain current $\left(\mathrm{I}_{\mathrm{d}}\right)$ on gate voltage $\left(\mathrm{V}_{\mathrm{g}}\right)$ was examined as shown in figure 3.7. The field effect mobility $(\mu)$ was calculated from the slope of the linear region of the curve in figure 3.7 by using the expression;

$$
\mu=\frac{\Delta I_{d}}{\Delta V_{g}} \frac{L}{W V_{d} C}
$$

where $V_{d}$ is the drain voltage $(0.5 \mathrm{~V}), C$ is the gate capacitance per unit area $\left(\frac{\varepsilon_{o x}}{t_{o x}}\right)$, and $L$ and $W$ are the channel length $(20 \mu \mathrm{m})$ and width $(100 \mu \mathrm{m})$ respectively. This device produced a mobility of about $1 \mathrm{~cm}^{2} / \mathrm{V} \cdot \mathrm{s}$, which is relatively low, due in part to the large channel length. Others have reported electron mobilities of $17 \mathrm{~cm}^{2} / \mathrm{V} \cdot \mathrm{s}$ with CVD grown 
bilayer $\mathrm{MoS}_{2}$ using shorter channel lengths [56]. Some have even reported very high electron mobility $>200 \mathrm{~cm}^{2} / \mathrm{V} \cdot \mathrm{s}$ with top gated transistors and $\mathrm{HfO}_{2}$ gate dielectric [17]. Our device has a lower electron mobility most likely due to a much larger channel length, hence more Coulombic scattering from surface impurities on the channel. One of the early samples of a bulk $\mathrm{MoS}_{2}$ film and a thick $(930 \mathrm{~nm})$ silicon oxide, had an electron mobility of $\sim 12 \mathrm{~cm}^{2} / \mathrm{V} \cdot \mathrm{s}$ for a channel length of $6 \mu \mathrm{m}$ and channel width of $100 \mu \mathrm{m}$. The contacts were made from $30 \mathrm{~nm} \mathrm{Au}$ on top of $30 \mathrm{~nm} \mathrm{Ti}$, and exhibited Ohmic contact.

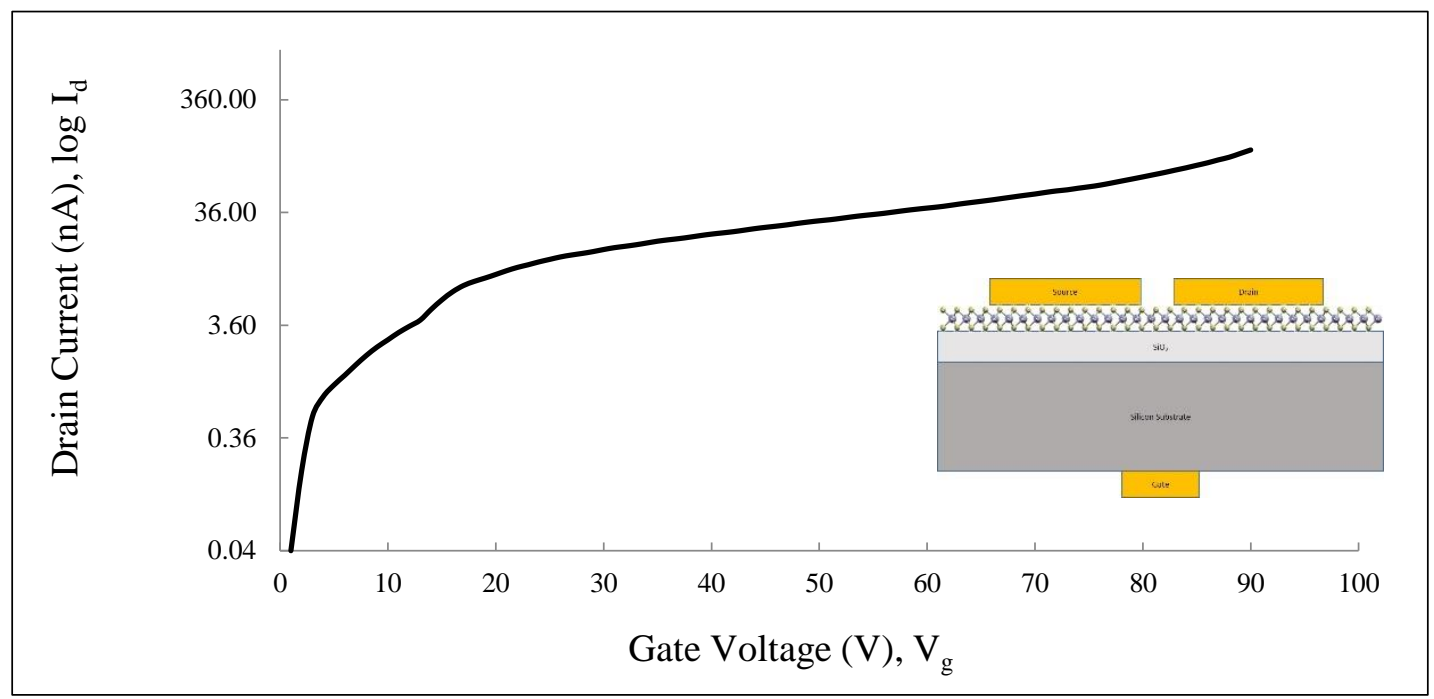

Figure 3.7: $\log I_{d}$ vs. $V_{g}$ plot of the back-gated $\mathrm{MoS}_{2}$ transistor [28]

An additional experiment was performed on one of the thicker films of $\mathrm{MoS}_{2}$, to investigate a possible phase transformation from $2 \mathrm{H}$ to $1 \mathrm{~T}$. $\mathrm{MoS}_{2}$ has three polytypes, as previously discussed, the $2 \mathrm{H}$ and $3 \mathrm{R}$ polytypes which are semiconducting and naturally occurring, and the $1 \mathrm{~T}$ polytype which is metallic and unstable. The large interlayer 
separation in $\mathrm{MoS}_{2}$ provides good locations for metal ions such as $\mathrm{Li}^{+}, \mathrm{Na}^{+}$, and $\mathrm{K}^{+}$ through a process known as intercalation. The intercalation of layered materials such as graphite and $\mathrm{MoS}_{2}$ have been studied for applications in energy storage and electrocatalysis. In one of the thicker $\mathrm{MoS}_{2}$ films we performed lithium intercalation to see if we could shift our $\mathrm{MoS}_{2}$ sample from semiconducting to metallic. A $1 \mathrm{M}$ lithium nitrate $\left(\mathrm{LiNO}_{3}\right)$ aqueous solution was prepared and 1-10 $\mu \mathrm{L}$ of solution were placed with a dropper on the $\mathrm{MoS}_{2}$ film between the source and drain contacts. After five minutes, the drain current vs. drain voltage measurement was taken and the data plotted in figure 3.8. Prior to intercalation the drain current was low, on the order of $10^{-10} \mathrm{~A}$. After the $\mathrm{Li}$ intercalation, the drain current increased $10^{5}$ for the same input voltage, indicating a possible change from semiconducting to metallic. Recent work shows large reduction in sheet resistance when Li intercalation was performed on $\mathrm{MoS}_{2}$ flakes [57]. Our data also shows a large change in resistance, based upon the change in drain current, after Li intercalation of the ALD grown $\mathrm{MoS}_{2}$ film. Band structure and density of states calculations, performed by others, show that the gap between the conduction and valence bands comes together to contact at the $K$ point (see figure 3.9), at which point the film becomes metallic $[58,59]$. 


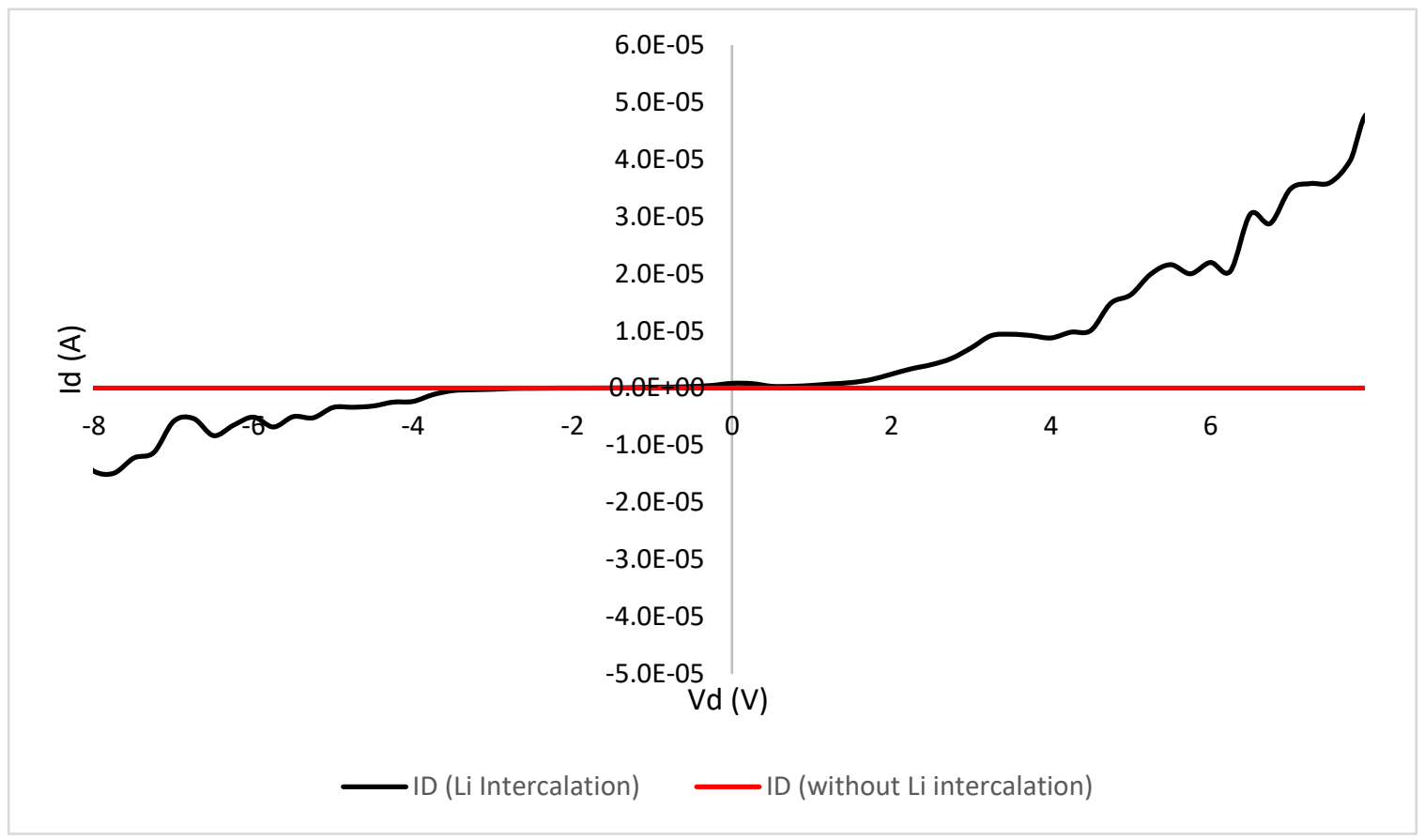

Figure 3.8: $\mathrm{MoS}_{2} I_{d^{-}} V_{d}$ with and without Li intercalation

The experimental data of our bi-layer $\mathrm{MoS}_{2}$ sample exhibits data consist with findings from other work with exfoliated flakes and CVD grown films. The electron mobility was relatively low due to Coulombic scattering by surface impurities from the large channel, however we have demonstrated that ALD grown $\mathrm{MoS}_{2}$ films possess the same electrical properties. Low resistance contacts using the low work function metal $\mathrm{Ti}$, exhibited an Ohmic contact, which is important for better electron injection and improved device performance. Further experimental data with Li intercalation exhibits a possible phase change which can be useful in energy storage applications. 


\subsection{WSe2 Electrical Data}

The electrical properties of the ALD grown $\mathrm{WSe}_{2}$ films were examined and shown to be much improved over those of $\mathrm{MoS}_{2}$. The transistors generally had sharper

turn on and higher $\mathrm{I}_{\mathrm{on}} / \mathrm{I}_{\text {off }}$ ratios than the $\mathrm{MoS}_{2}$ transistors. The $\mathrm{WSe}_{2}$ back-gated FETs were created with varying channel lengths like those shown in figure 3.2. Several devices were fabricated with various metals for the contacts, to characterize the electrical properties of $\mathrm{WSe}_{2}$ with each metal. The formation of low resistance metal contacts is one of the biggest challenges to creating high performance semiconducting devices to take advantage of the exceptional electronic properties of the TMD films. For this reason, several metals were chosen to test with $\mathrm{WSe}_{2}$ films. Since $\mathrm{WSe}_{2}$ is a p-type material, metals with high work functions close to the valence band of $\mathrm{WSe}_{2}$ would create lower resistance contacts due to lower Schottky barrier heights. We also tested lower work function metals to understand the n-type characteristics of the film. The metals used were $\mathrm{Ni}, \mathrm{Au}, \mathrm{Pd}$, and Ti, deposited via electron beam deposition each about $30 \mathrm{~nm}$ thick. Silicon oxide thickness was between about 30 to $60 \mathrm{~nm}$ allowing for a much smaller gate voltage to turn on and control the device. Channel lengths were 2 to $10 \mu \mathrm{m}$ with a channel width of $100 \mu \mathrm{m}$.

After the devices had metal deposited, a series of electrical tests were then performed. Since the insulating silicon oxide layer was very thin, $I_{d^{-}} V_{d}$ profiles between top source/drain electrodes and back gate electrode were performed to determine onset of leakage current for each device. Subsequent gate voltages were kept far below this value to prevent excessive gate leakage. Source to drain $I_{d^{-}} V_{d}$ profiles were collected with no 
applied gate bias, to determine the type of contact with each metal. Most of the metals exhibited Schottky type contact, as expected, due to Fermi level pinning at the semiconductor metal interface, however a few samples exhibited Ohmic contact behavior when Au was used (see Fig. 3.9). A few samples were annealed with selenium after the metallization process to lower the contact resistance to determine any effect on carrier mobility. The samples were annealed in a Se vapor at about 5 Torr for 1.5 hours at 500 ${ }^{\circ} \mathrm{C}$. One of the samples with Ni contacts exhibited Ohmic behavior after the annealing process, as shown in figure 3.10, while the sample with Pd contacts still exhibited Schottky behavior, albeit with higher current after the anneal. The interfaces between contact metals and 2D materials generally have some Schottky barrier, however the post metallization annealing is believed to have changed the interface between the metal and the semiconductor film allowing for Ohmic contact $[60,61]$. The drain current shown in figure 3.10 is clearly higher after the anneal process indicating a lower resistance. Unlike the high work function metals, when low work function Ti was used, strong Schottky behavior was exhibited as expected [54]. Figure 3.9 shows the $I_{d}-V_{d}$ profile of a few devices with the different contact metals. The Ohmic behavior seen with Au contacts is due to the high work function of $\mathrm{Au}(\sim 5.4 \mathrm{eV})$ being close to the valence band of $\mathrm{WSe}_{2}$, creating a small Schottky barrier and lowering resistance. When Pd and Ni were used as the contact metal, a slight Schottky behavior was exhibited. The work functions for Ni $(\sim 5.04 \mathrm{eV})$ and $\mathrm{Pd}(\sim 5.22 \mathrm{eV})$ are relatively close to each other but lower than $\mathrm{Au}$, and further away from the $\mathrm{WSe}_{2}$ valence band, which explains the slight Schottky behavior. 
The strong Schottky behavior seen with $\mathrm{Ti}$ is due to its much lower work function $(\sim 4.3$ $\mathrm{eV}$ ) compared to the other metals used.
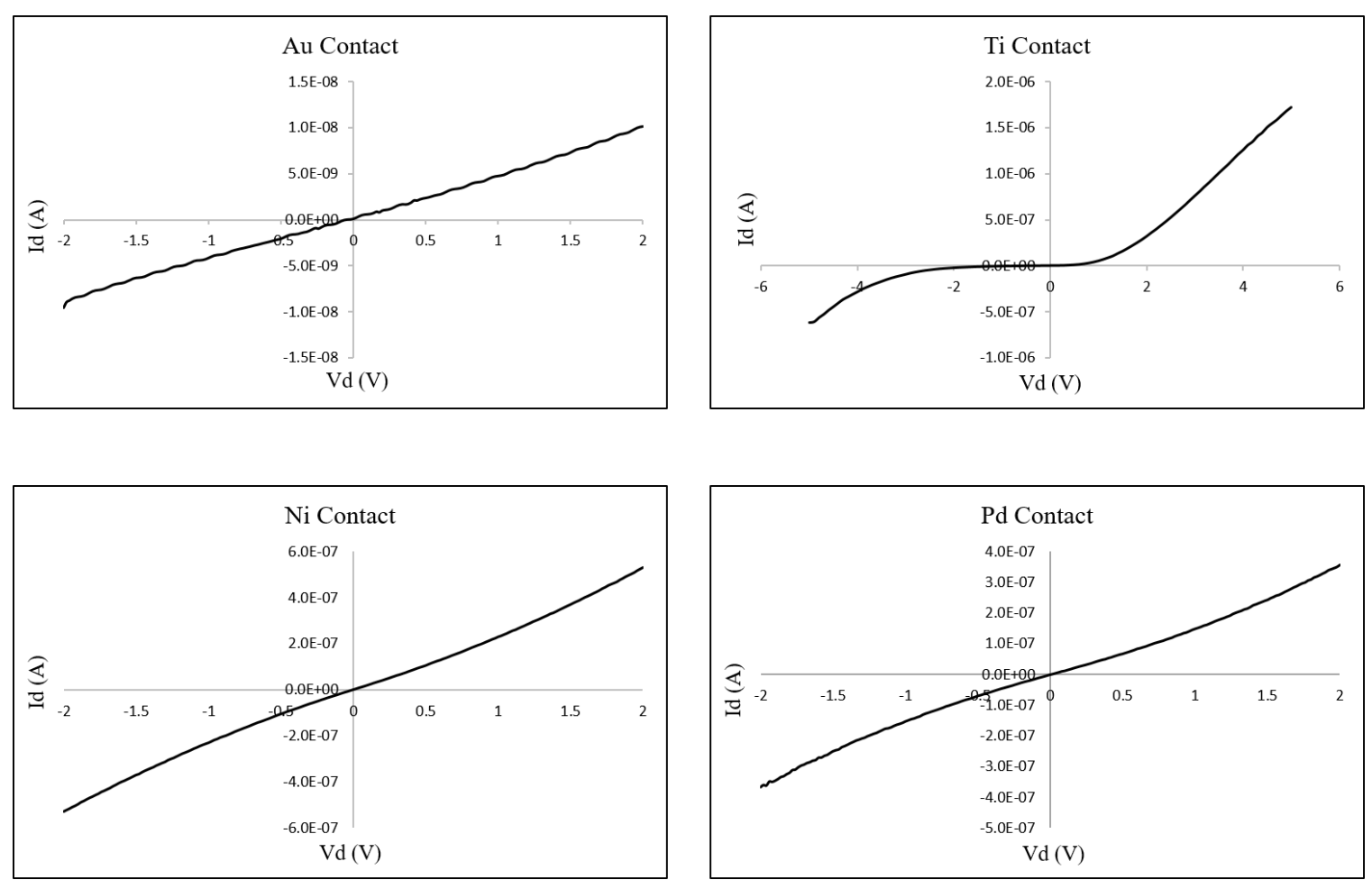

Figure 3.9: $I_{d^{-}} V_{d}$ profiles for different metal contacts with WSe $[41]$. 


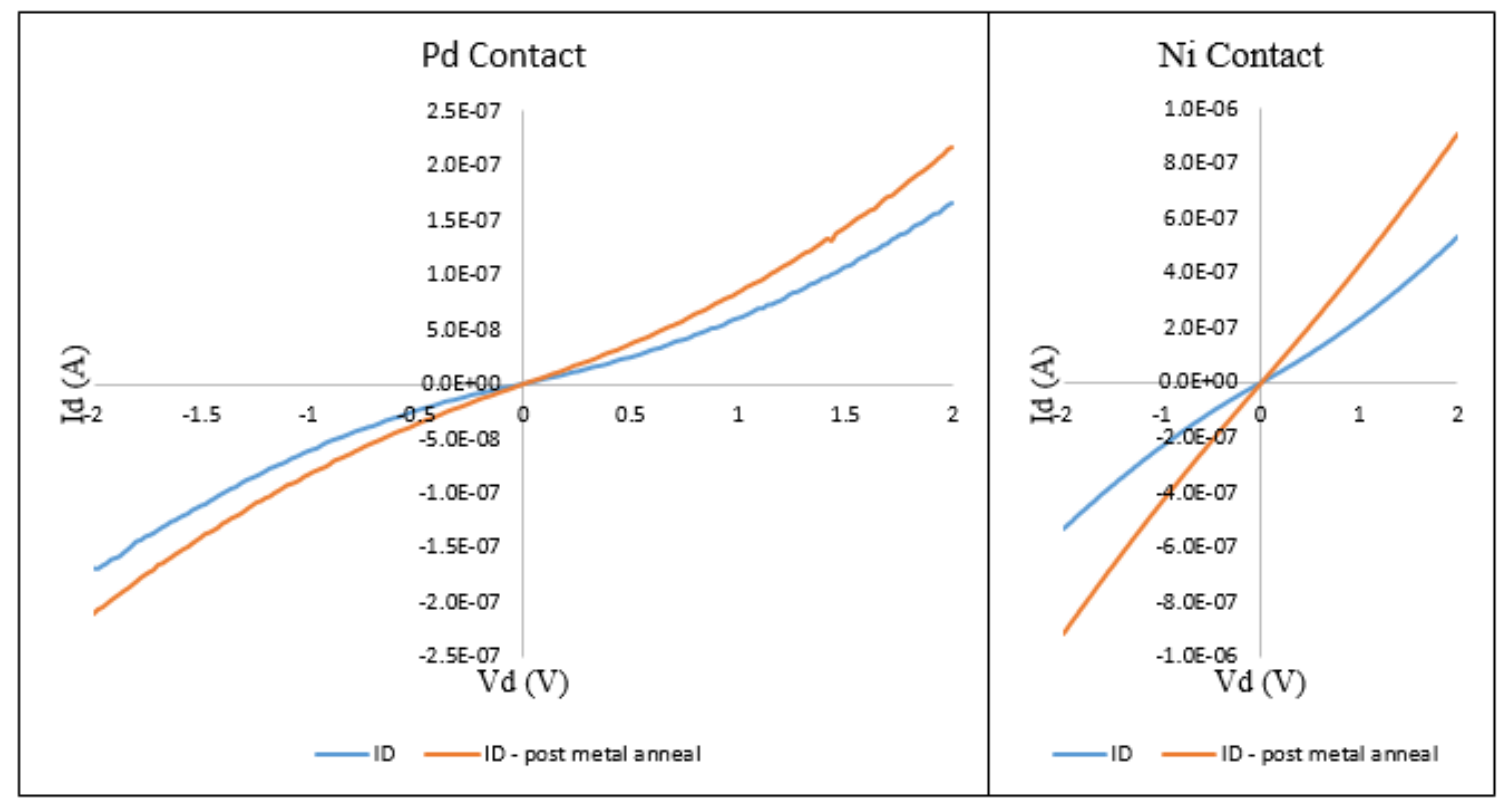

Figure 3.10: $I_{d}-V_{d}$ profile of $P d$ and Ni contacts before and after post-metallization anneal. $I_{d}$ increases for the same $V_{d}$ indicating lower resistance post metal anneal.

Further analysis of the metal-semiconductor junction was performed by calculating the contact resistance of the metal to the semiconductor film. To perform this calculation, we consider the geometry of a simple resistor that has two contacts, one at each end of the resistor. The total resistance consists of the resistances of the two metal contacts $\left(\mathrm{R}_{\mathrm{m}}\right)$, the resistance of the two metal/semiconductor interfaces $\left(\mathrm{R}_{\mathrm{C}}\right)$, and the resistance of the semiconductor $\left(\mathrm{R}_{\mathrm{semi}}\right)$. The equation would for this would be; $R_{T}=2 R_{m}$ $+2 R_{c}+R_{\text {semi }}$. Since the resistance of the metal is so low compared to the contact resistance, $R_{m}$ can be ignored. The semiconductor resistance is; $R_{\text {semi }}=R_{S} \frac{L}{W}$, or after 
substitution, $R_{T}=\frac{R_{S}}{W} L+2 R_{C}$. If resistors of several different lengths are constructed, and keeping all other dimensions the same, the total resistance can be measured and plotted. If we take the limit as L goes to zero (zero length resistor), the remaining resistance will be just twice the contact resistance, which can be found from the graph of figure 3.13 by extrapolating L back to zero. The measurements were taken using one of the transfer length method (TLM) structures shown on the right side of the devices as seen in figure 3.2, and using the equations (3.2) for TLM calculations.

$$
\begin{aligned}
& R c=\frac{\mathrm{y} \text {-intercept }}{2} \\
& R_{S}=\text { slope } \times W \\
& L_{T}=\frac{\mathrm{y} \text {-intercept }}{2(\text { slope })} \\
& \rho_{c}=R_{C} L_{T} W
\end{aligned}
$$

$R_{C}$ is the contact resistance of the metal contact to the semiconductor film, $R_{S}$ is the sheet resistance of the semiconductor film, $W$ is the width of the TLM contact (500 $\mu \mathrm{m}$ in our sample), $L_{T}$ is the transfer length (average distance an electron or hole travels in the semiconductor underneath the contact before it flows up into the contact), and $\rho_{c}$ is the contact resistivity between the contact and the semiconductor. Drain voltage was swept from 0 to $5 \mathrm{~V}$ while drain current was measured between two adjacent contacts of the TLM structure (Fig. 3.11) and plotted on the $I_{d}$ - $V_{d}$ curve shown in figure 3.12. 


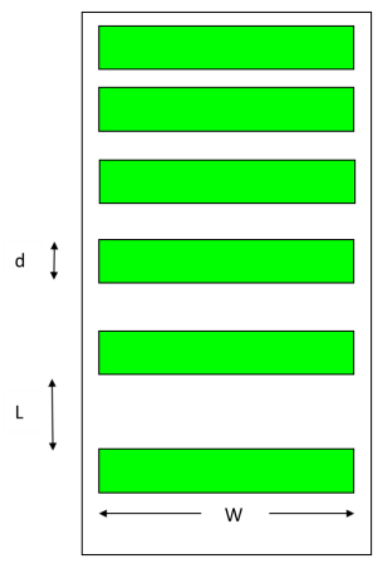

Figure 3.11: Drawing of TLM structure from figure 3.2

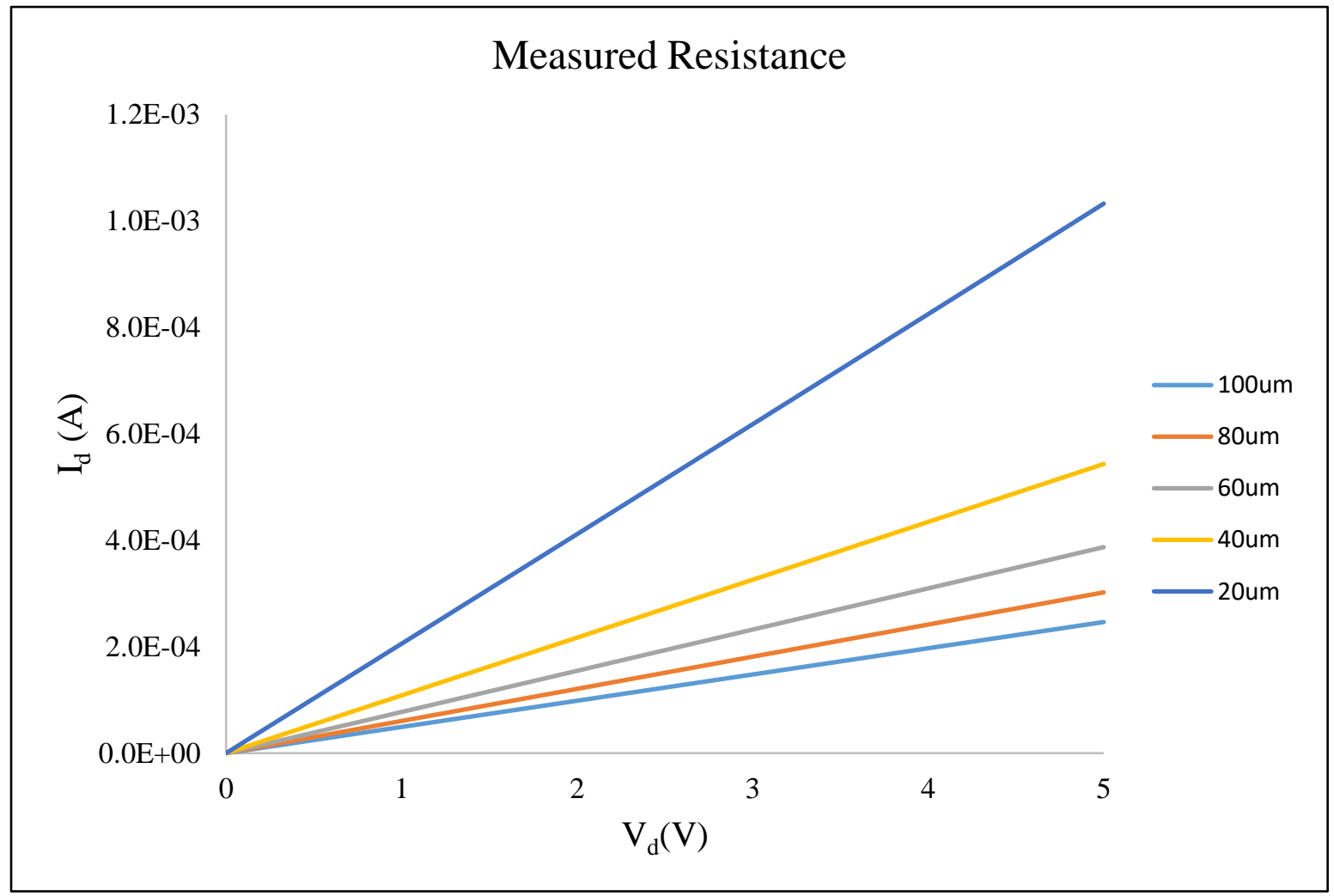

Figure 3.12: Measured resistance between the Au contacts of the TLM structure for WSe 2 
Resistance between each TLM contact is the inverse slope of the curve of each line shown in figure 3.12. The resistance is then plotted vs. distance (dimension L in Fig. 3.11) between the TLM contacts (Fig. 3.13). The y-intercept (circled in red) of the plot is twice the contact resistance.

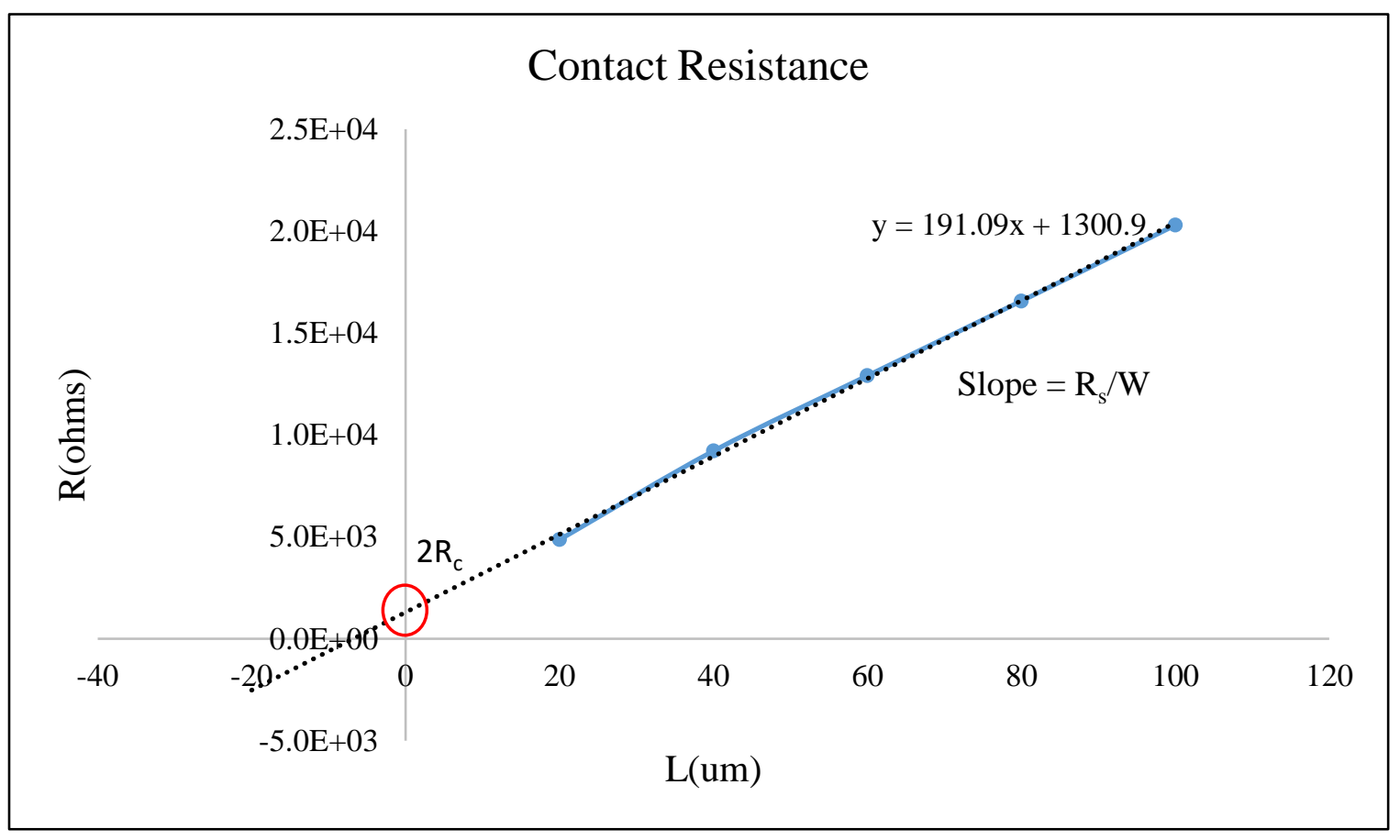

Figure 3.13: WSe 2 contact resistance plot

The resistance measurement results from one of the $\mathrm{WSe}_{2}$ samples with Au contacts are shown in figure 3.13 . The channel lengths were created to be $20,40,60,80$, and $100 \mu \mathrm{m}$. These resistances were then plotted as shown in figure 3.13 and a trend line of those points plotted. The slope of the line is $\frac{R_{s}}{W}$, which also gives the sheet resistance of the 62 
semiconductor film as a secondary benefit. From the plot in figure 3.13, it was found that the contact resistance for Au was about 650 ohms, which is a contact resistivity $\left(\rho_{\mathrm{c}}\right)$ of $1.1 \times 10^{-2} \Omega \cdot \mathrm{cm}^{2}$. These same measurements were performed with $\mathrm{Ni}$ and $\mathrm{Pd}$ and measured to be about $150 \mathrm{k} \Omega$ and $27 \mathrm{k} \Omega$, or $\rho_{\mathrm{c}}$ equal to $4.4 \Omega \cdot \mathrm{cm}^{2}$ and $0.27 \Omega \cdot \mathrm{cm}^{2}$ respectively. The higher resistance values for Ni and Pd clearly explain the Schottky behavior in those two metal contacts, while Au has a much more Ohmic behavior due to the lower contact resistance. If these were Schottky contacts, then the $I$ vs $V$ plots of figure 3.12 would not be linear.

Next, the dependence of the drain current on the gate voltage was examined. The drain current versus gate voltage profile of a device of gate length $2 \mu \mathrm{m}$ with Pd electrodes is shown in figure 3.14. Using a drain voltage of $0.1 \mathrm{~V}$ and sweeping the gate voltage, the forward and reverse gate response shows an ambipolar characteristic, which has been seen using Pd and $\mathrm{Ni}$ as contact metals $[62,63]$. This is the same sample whose TEM profile is shown in figure 2.10. The effective mobilities $(\mu)$ were calculated from the linear plot of this curve using equation 3.1. The hole and electron mobilities for this device were determined to be $354 \mathrm{~cm}^{2} / \mathrm{V} \cdot \mathrm{s}$ and $531 \mathrm{~cm}^{2} / \mathrm{V} \cdot \mathrm{s}$ respectively. The mobility values for this device were found to be the median values of all the devices tested, and the hole mobility is comparable to that reported for CVD grown and exfoliated fewlayered $\mathrm{WSe}_{2}$ films $[64,65]$. When the Au electrodes were used, the device turn-on was not as sharp, and the hole and electron mobilities were determined to be slightly lower. Although the contact was Ohmic, Pd contacts have lower tunnel and Schottky barriers than $\mathrm{Au}[54,66]$. In the case of the $\mathrm{Ti}$ and $\mathrm{Ni}$ electrodes, the currents were in the $\mu \mathrm{A}$ 
range and the carrier mobilities were much lower: the electron mobilities were 21 (22) $\mathrm{cm}^{2} / \mathrm{V} \cdot \mathrm{s}$ and the hole mobilities were $5(17) \mathrm{cm}^{2} / \mathrm{V} \cdot \mathrm{s}$ for $\mathrm{Ti}(\mathrm{Ni})$. The drain current versus gate voltage profile for the device with Ni contacts, shown in figure 3.15, had a gate length of $6 \mu \mathrm{m}$ and an insulating layer of silicon oxide $320 \mathrm{~nm}$ thick. The curve shifted slightly to the left with the Ni contacts as compared to the device with Pd contacts because Ni favors electron injection. The ambipolar transfer characteristics in these devices are similar to $\mathrm{WSe}_{2}$ FETs created from exfoliated flakes with $\mathrm{Ni}$ as the source and $\mathrm{Pd}$ as the drain contact [63].

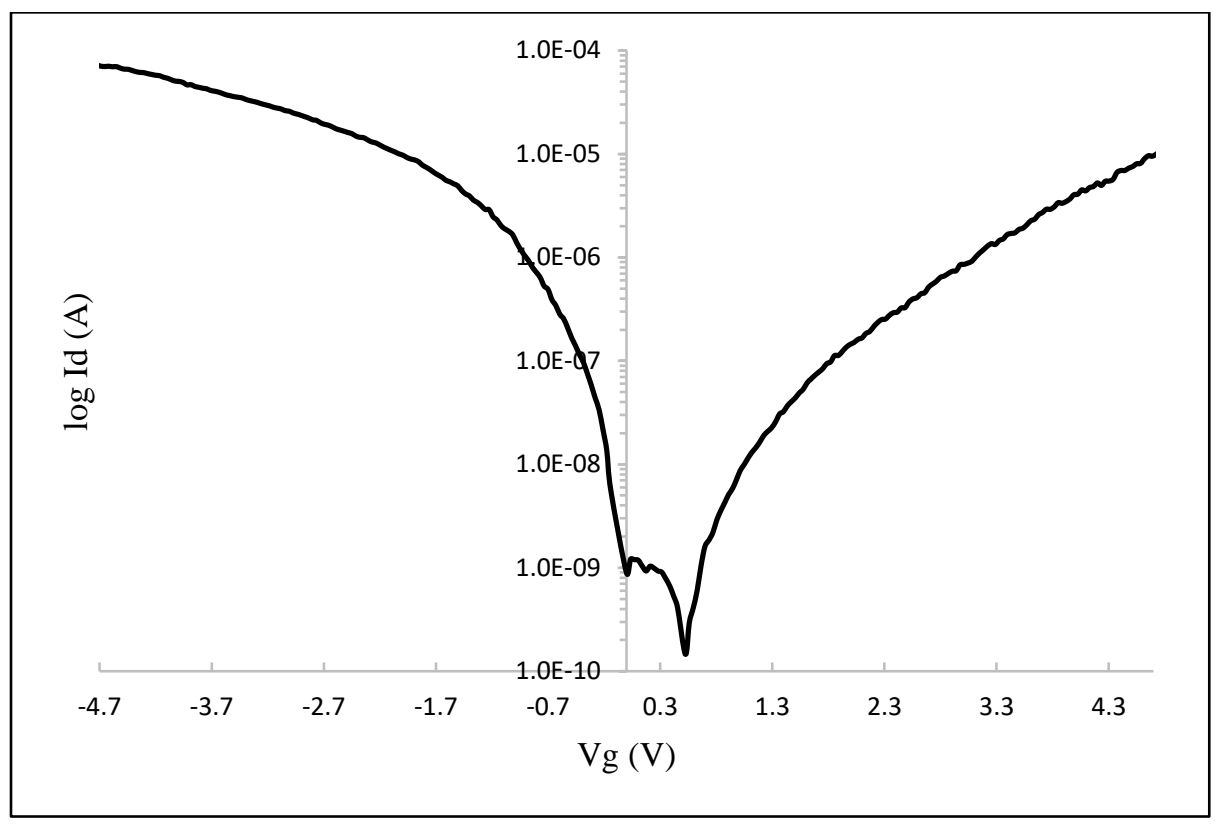

Figure 3.14: Plot of the field effect current vs. the back-gate voltage showing ambipolar behavior with Pd contacts [41] 


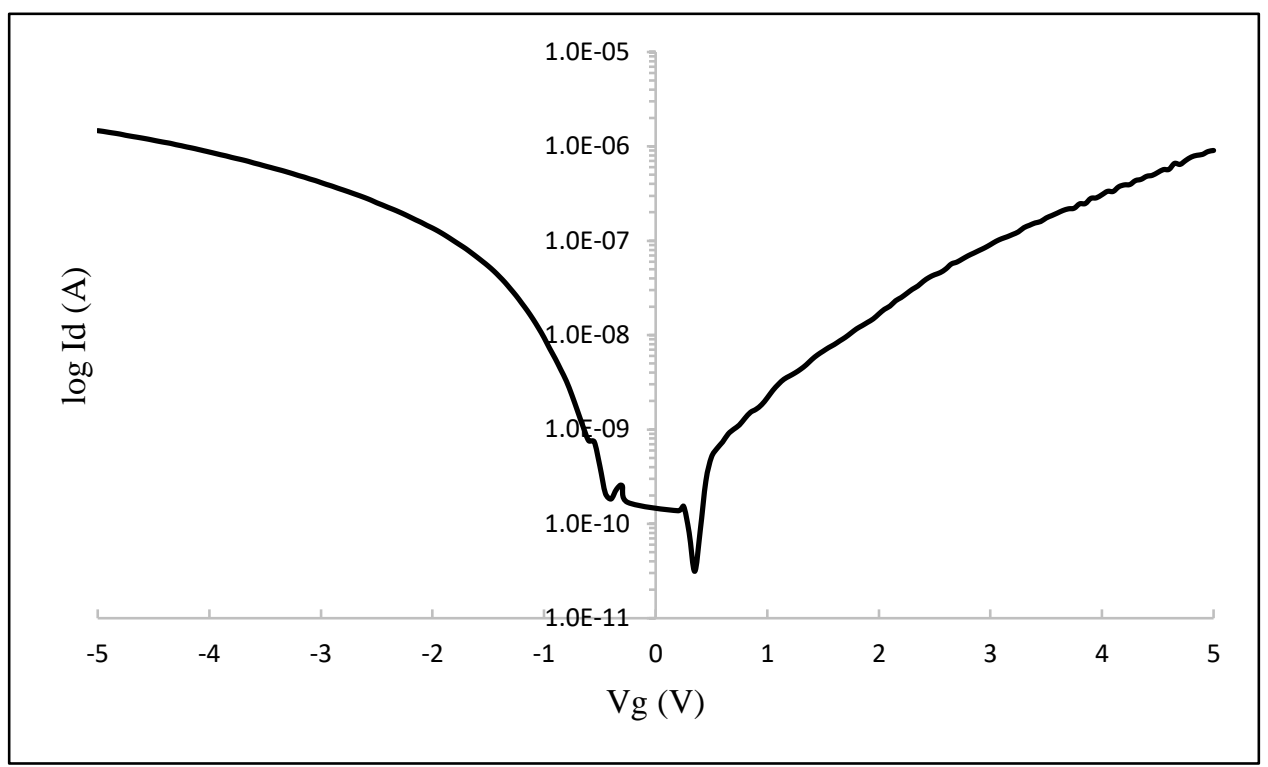

Figure 3.15: Plot of the field effect current vs. the back-gate voltage with Ni contacts

The electrical performance of our $\mathrm{WSe}_{2}$ devices was exceptional, and shows the potential for creation of good quality semiconductor devices using ALD. The $\mathrm{WSe}_{2}$ devices exhibited large on/off ratios, high field effect mobilities and large current carrying capacity. Use of the proper contact metal is vitally important as seen in the contact resistance and mobility values exhibited with the various metals.

\subsection{WS2/SnS Heterostructure Electrical Data}

To examine the electrical characteristics of the $\mathrm{WS}_{2} / \mathrm{SnS}$ heterostructure, we first examine the electrical data for $\mathrm{WS}_{2}$ and $\mathrm{SnS}$ individually. The $\mathrm{WS}_{2}$ film was grown via ALD, as previously described, to be $2.5 \mathrm{~nm}$ thick, or approximately 3 atomic layers. The Raman spectrum for this device is shown in figure 2.13. The film was grown on top of a 
layer of silicon oxide $\sim 930 \mathrm{~nm}$ thick. Optical lithography was used to pattern source and drain contacts, like that in figure 3.2. Contacts were created by electron beam (sputtering) deposition of $30 \mathrm{~nm}$ of Ti followed by $30 \mathrm{~nm}$ of Au. Since $\mathrm{WS}_{2}$ is an n-type material, the low work function metal Ti was deposited as the contact. Au was put on top of Ti to prevent oxidation. Ag was used for the wafer backside contact as the gate bias, creating back-gated field effect transistors. With the gate bias set to zero, $I_{d}-V_{d}$ profiles were recorded and plotted in figure 3.16. The curve is a near-linear profile, indicating Ohmic contact.

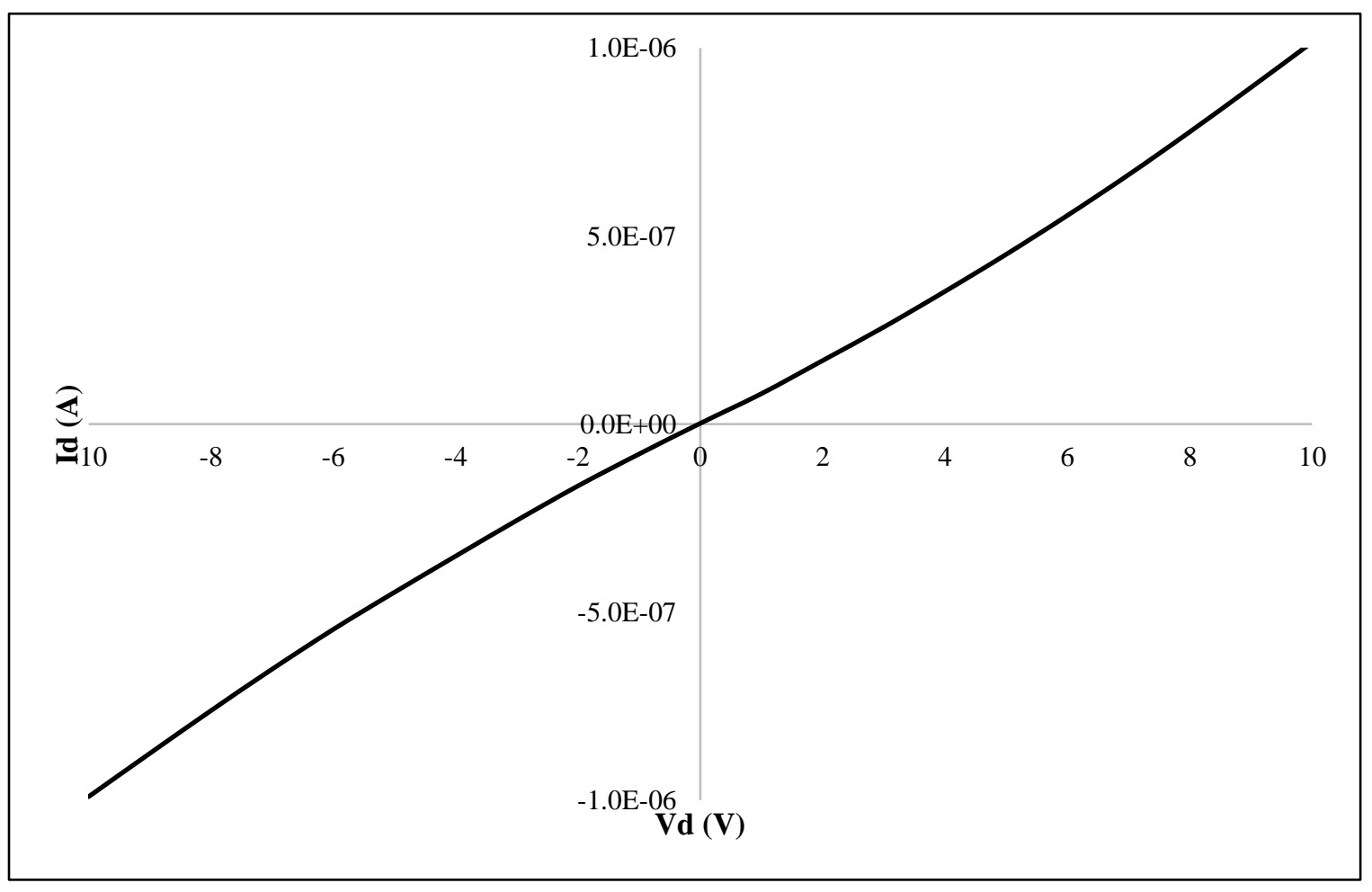

Figure 3.16: $I_{d}-V_{d}$ curve of $W_{2}$ with Ti contacts 
Next, the dependence of the drain current $\left(\mathrm{I}_{\mathrm{d}}\right)$ on the gate voltage $\left(\mathrm{V}_{\mathrm{g}}\right)$ was examined and data plotted in figure 3.17. The data shows nearly $10^{5} \mathrm{I}_{\mathrm{on}} / \mathrm{I}_{\mathrm{off}}$ ratio. The field effect mobility was extracted from the linear region of the curve using the equation 3.1, where $V_{d}$ is the drain voltage $(0.1 \mathrm{~V}), C$ is the gate capacitance per unit area, and $L$ and $W$ are the channel length and width, $6 \mu \mathrm{m}$ and $100 \mu \mathrm{m}$ respectively. The $\mathrm{WS}_{2}$ transistor with Ti/Au contacts, which is dominated by electrons, displayed an n-type transport with mobility of $12 \mathrm{~cm}^{2} / \mathrm{V} \cdot \mathrm{s}$.

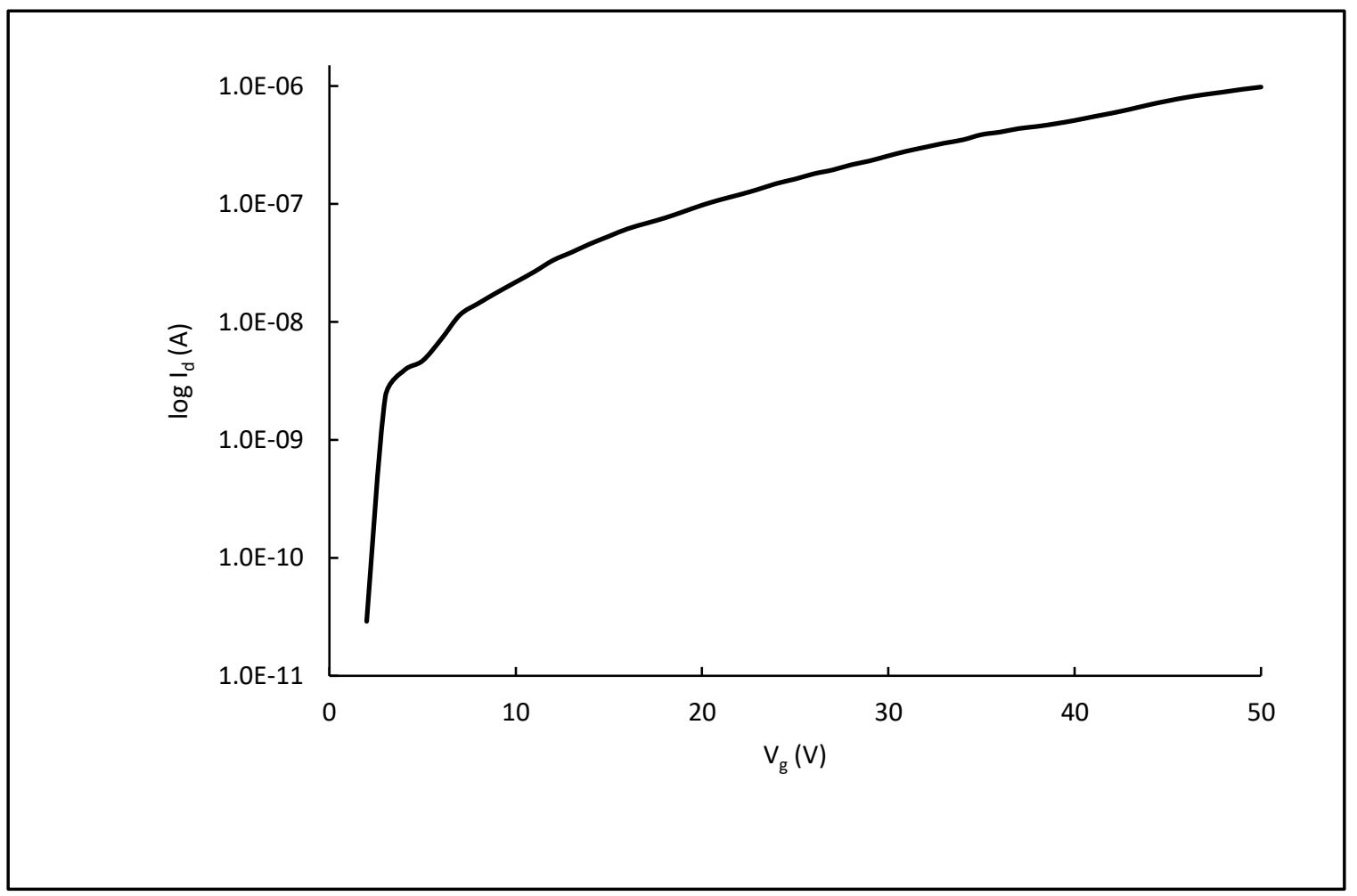

Figure 3.17: Transfer characteristics of a $W_{2}$ FET [47] 
SnS was also independently grown, via ALD, on top of a layer of silicon oxide 55 nm thick, and source and drain contacts were created using optical lithography. Contacts were created by electron beam deposition of $30 \mathrm{~nm}$ thick $\mathrm{Au}$, and $\mathrm{Ag}$ was used for backside metal contact. Au was used as the contact metal because $\mathrm{SnS}$ is a p-type semiconductor and so metals with high work functions are required to form Ohmic contacts. With the gate bias set to zero, the $I_{d}-V_{d}$ profile was recorded and plotted in figure 3.18 and exhibits a strong Schottky contact. The drain current versus gate voltage was then examined and plotted in figure 3.19, which exhibits p-type behavior of SnS. The gate length (L) and width (W) was $6 \mu \mathrm{m}$ and $100 \mu \mathrm{m}$ respectively, while the drain voltage $\left(\mathrm{V}_{\mathrm{d}}\right)$ was $0.3 \mathrm{~V}$. The plot shows an $\mathrm{I}_{\mathrm{on}} / \mathrm{I}_{\text {off }}$ ratio of nearly $10^{6}$, and an effective field effect mobility of $818 \mathrm{~cm}^{2} / \mathrm{V} \cdot \mathrm{s}$ which was extracted from the slope of the curve in the linear region of the plot. These are among the highest values recorded for $\mathrm{SnS}$ films. Ion $/ \mathrm{I}_{\text {off }}$ ratios of $10^{6}$ are well suited for the next generation FET devices since the desired $\mathrm{I}_{\mathrm{on}} / \mathrm{I}_{\mathrm{off}}$ ratio for any silicon replacement FET is from $10^{4}$ to $10^{7}$ [67]. An $\mathrm{I}_{\mathrm{on}} / \mathrm{I}_{\text {off }}$ ratio of $10^{6}$ demonstrates that the off-state current is low compared to the on-state current, signifying less current leakage in the off-state and higher performance when the transistor is turned on. This gives more control over the gate and therefore better transistor performance. 


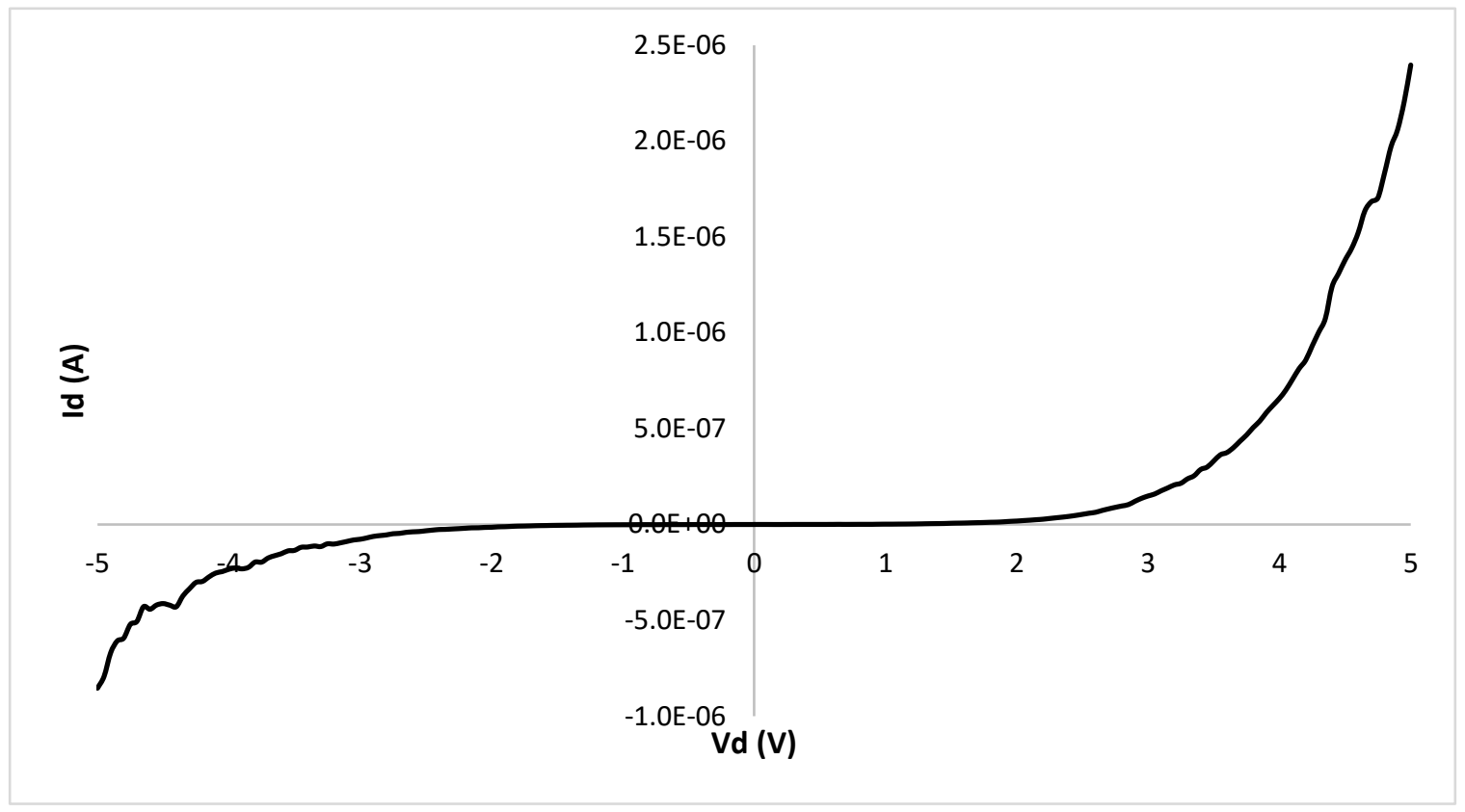

Figure 3.18: $I_{d^{-}} V_{d}$ curve of $S n S$ with Au contacts 


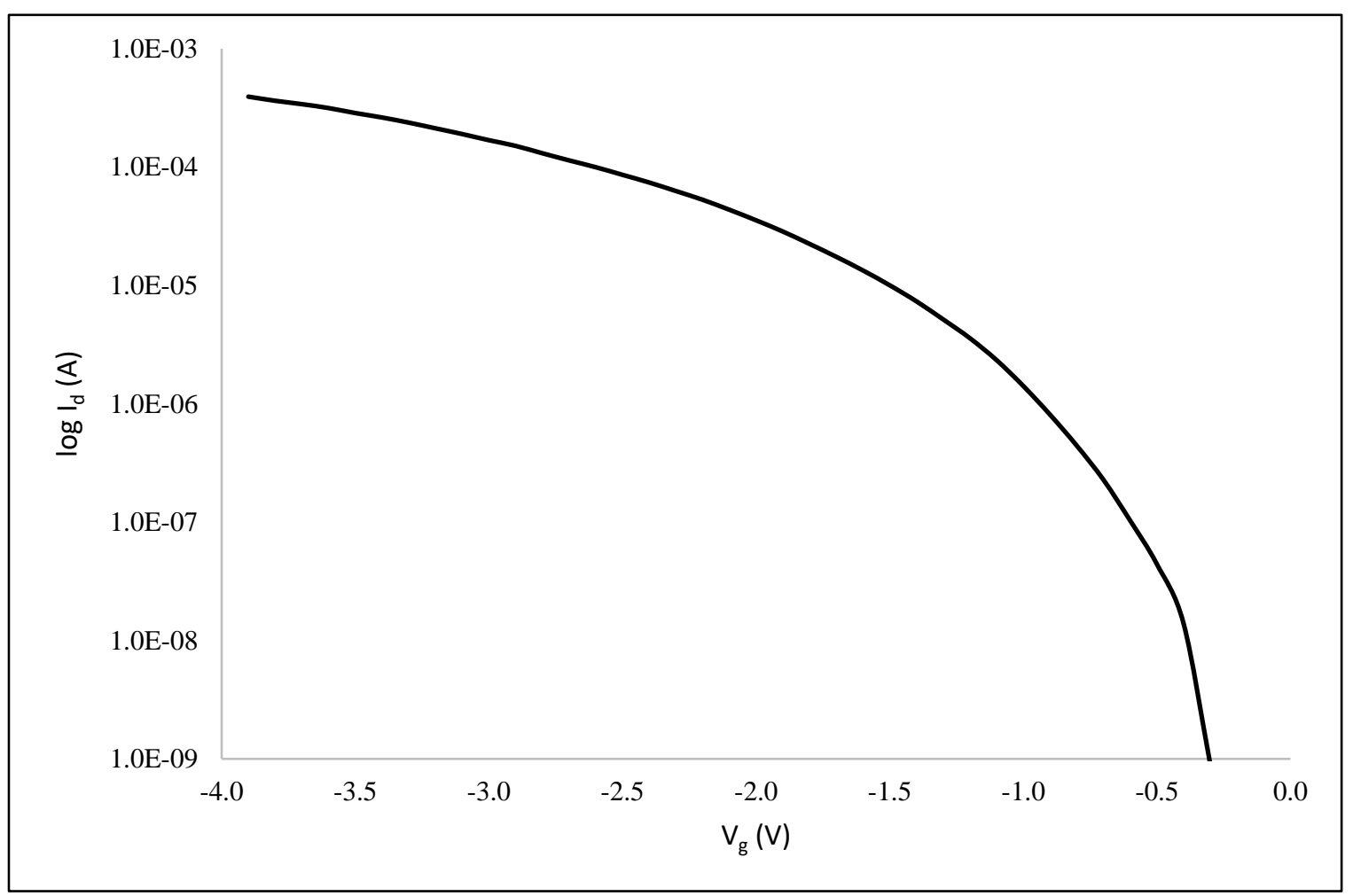

Figure 3.19: Transfer characteristics of an SnS FET [47]

After characterizing the individual films $\mathrm{WS}_{2}$ and $\mathrm{SnS}$, the electrical characteristics of the heterostructure film of six layers alternating 3 layers each of $\mathrm{WS}_{2}$ and $\mathrm{SnS}$ (Fig. 2.16) were examined. $\mathrm{WS}_{2}$ and $\mathrm{SnS}$ were grown via $\mathrm{ALD}$, as described in section 2.5, on a layer of silicon oxide $\sim 360 \mathrm{~nm}$ thick. Back-gated FETs were created using the same process as previously described, and the contacts were made with $30 \mathrm{~nm}$ of $\mathrm{Au}$ via electron beam deposition. Au was used as the contact metal because the top layer of the heterostructure was SnS. Since SnS is a p-type material, metals with a high work function close to the valence band of $\mathrm{SnS}$, such as $\mathrm{Au}$, are needed to reduce the 
Schottky barrier and allow for improved hole transport. With the gate bias set to zero, the $I_{d^{-}} V_{d}$ curve was plotted (Fig. 3.21), indicating Schottky contact.

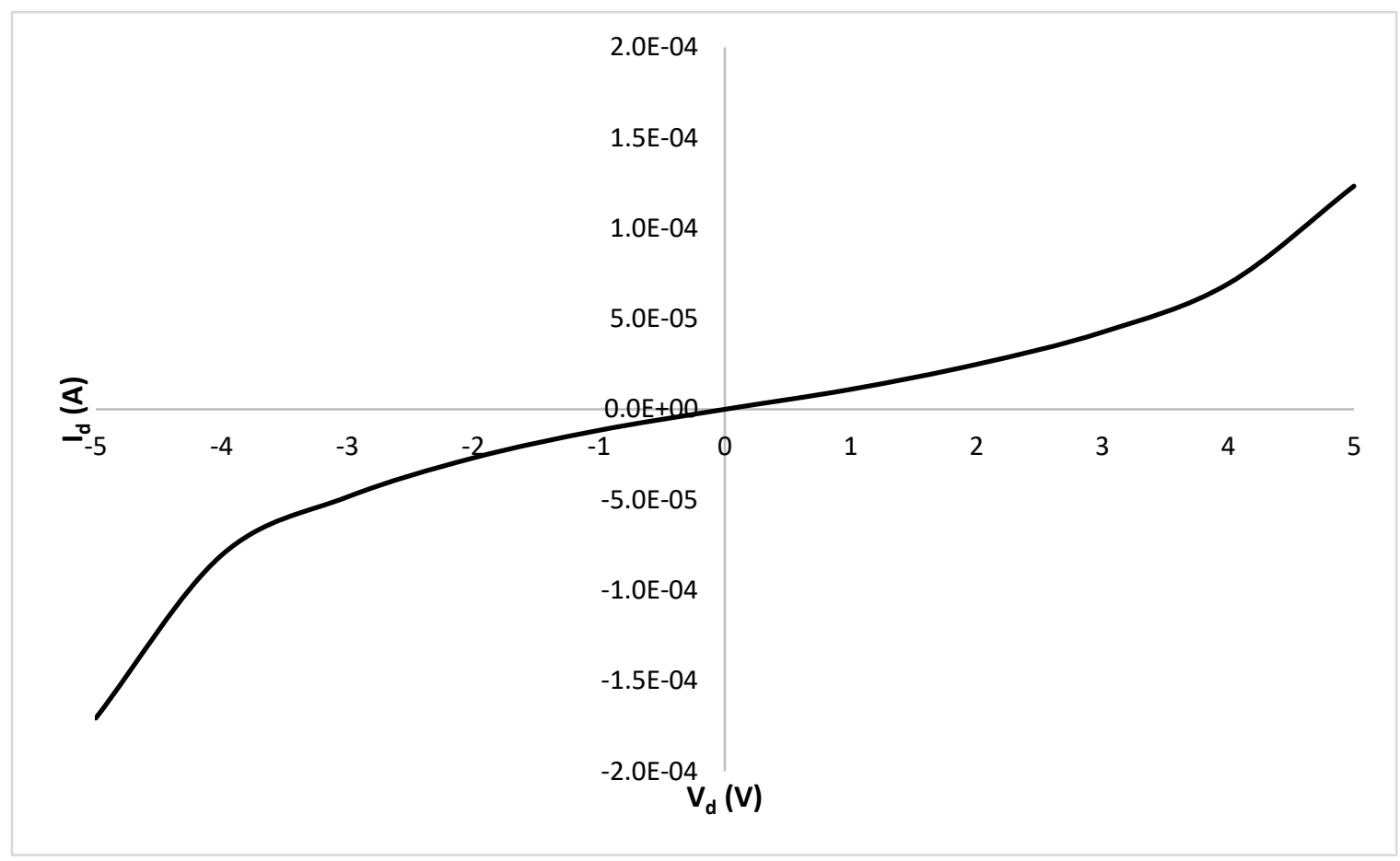

Figure 3.20: $I_{d}-V_{d}$ curve for $W S_{2} / S n S$ heterostructure

The drain current versus gate voltage was then examined and shows an ambipolar conductance (Fig. 3.21), which is not surprising based upon the results of the individual transistor performance. For the positive drain bias and gate voltage, it represents and nFET while for negative drain bias and gate voltage it represents a p-FET. The effective mobilities were $48 \mathrm{~cm}^{2} / \mathrm{V} \cdot \mathrm{s}$ for the n-FET and $20 \mathrm{~cm}^{2} / \mathrm{V} \cdot \mathrm{s}$ for the p-FET. There is an increase in the mobility of $\mathrm{WS}_{2}$ in the heterostructure as compared to the individual film, however the mobility of SnS drops. The significant drop in the $\mathrm{SnS}$ hole mobility is 
attributed to the resistance of hole transport through the layer boundaries of SnS due to its alignment with respect to $\mathrm{WS}_{2}$ as described in section 2.5. This is consistent with the reported significant decrease in mobility of holes traveling from parallel to perpendicular direction of the van der Waals layers $[8,48]$. The $I_{o n} / I_{o f f}$ ratio for this device was found to be $10^{5}$, which is one of the highest recorded for heterostructure devices.

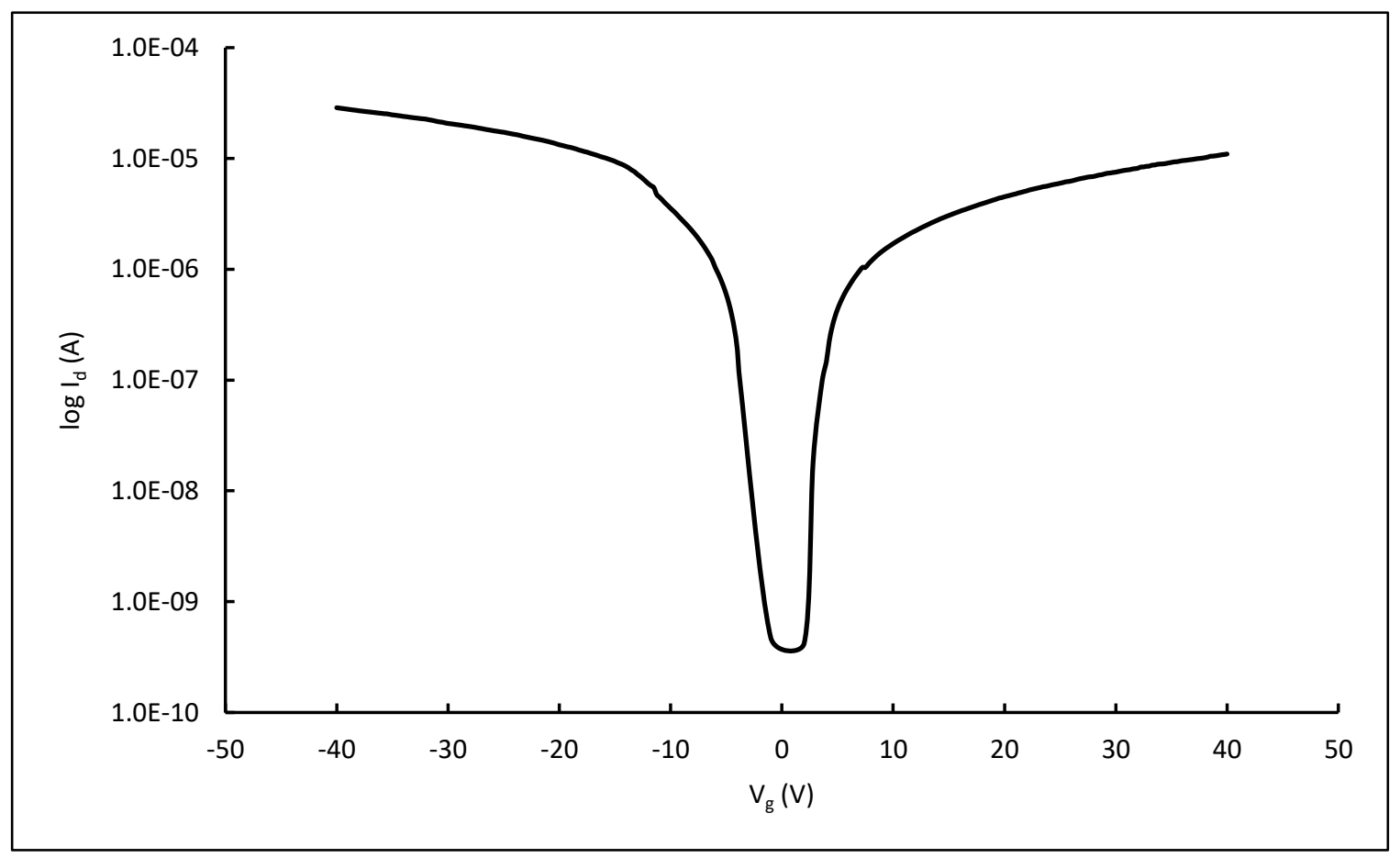

Figure 3.21: Transfer characteristics of the heterojunction FET [47]

\subsection{Summary}

The electrical characteristics of $\mathrm{MoS}_{2}, \mathrm{WSe}_{2}, \mathrm{WS}_{2}, \mathrm{SnS}$, and the heterostructure $\mathrm{WS}_{2} / \mathrm{SnS}$ have been examined to determine how the electrical properties of films grown 
via ALD compared to those created with other methods. Most of the electrical data collected in literature has been from exfoliated samples, with some limited data from other deposition or growth methods. The $\mathrm{MoS}_{2}$ electrical data collected used larger device dimensions than the later samples of $\mathrm{WS}_{2}, \mathrm{SnS}$, and $\mathrm{WSe}_{2}$, and therefore the device characteristics were less impressive, but still matched to literature. Due to the larger gate lengths, the impact of coulombic scattering limited the measured mobility of the $\mathrm{MoS}_{2}$ devices, however the electrical data shows that ALD grown $\mathrm{MoS}_{2}$ can create devices with suitable device performance.

$\mathrm{WSe}_{2}$ electrical data was an improvement over the $\mathrm{MoS}_{2}$ samples grown. These films had higher mobilities and larger $\mathrm{I}_{\mathrm{on}} / \mathrm{I}_{\mathrm{off}}$ ratios than the $\mathrm{MoS}_{2}$ films that were grown. The $\mathrm{WSe}_{2}$ films were grown on top of thin silicon oxide which allowed for smaller gate bias voltages to be used to control the device. Smaller channel lengths allowed for better response to signals and eliminated any fringing effects due to the high ratio of channel width to length. Several different metals were used to create the contacts of the $\mathrm{WSe}_{2}$ devices to determine which metal provided better contact. In our samples Au produced the lowest contact resistance, however the device performance using Pd as the contact metal was better than transistors with the Au contacts. This most likely was due to an interface covalent bond of $\mathrm{Pd}$ with $\mathrm{WSe}_{2}$ which reduces the Schottky barrier height and improves performance. Ni had Schottky contact as expected, but when an anneal step was performed after metallization, the contact became Ohmic, suggesting that a change of contact interface between the film and the metal or larger grain size of the metal film. One possible model for this change is that the bond between the metal and the film 
formed an interface covalent bond, reducing the resistance and making a more Ohmic contact. It has been shown that annealing after metal deposition on graphene reduces contact resistance which leads to Ohmic contacts [61]. Ti with its low work function had the strongest Schottky behavior, as expected. The $I_{d^{-}} V_{d}$ curves of the short channel devices (2 $\mu \mathrm{m}$ gate length) with Pd contacts had superior electrical performance over the other metal contacts. The devices with Ni contacts also provided good device performance, but the drain current was about one order of magnitude lower than devices with Pd contacts. Ni also shows improved electron injection and reduced hole injection but Pd clearly shows the better performance.

The electrical data for the individual films $\mathrm{WS}_{2}$ and $\mathrm{SnS}$ showed n-type and ptype behavior respectively. Although the high work function metal Au was used as the contact for $\mathrm{SnS}$, there was still a Schottky type contact to the $\mathrm{SnS}$ film. This was seen on the individual film as well as the heterostructure device. The field effect mobility of the $\mathrm{WS}_{2}$ film was much less than the $\mathrm{SnS}$ film, however when the films were combined, the SnS mobility was significantly reduced while the $\mathrm{WS}_{2}$ mobility increased, due to the carrier transport through the interface. Good ambipolar characteristics were seen from the device, which is needed to create n-FET and p-FET devices without the need for difficult doping methods.

Overall, the electrical characterization of several TMD films show that high quality devices made from atomically thin films using ALD, can be fabricated, and that the device performance is equal to or better than those reported in literature. 


\section{Chapter IV: Density Functional Theory}

\subsection{Introduction and Theoretical Background}

The structural and electronic properties of TMDs were investigated based upon

first principles calculations within the framework of density functional theory (DFT), and then compared to the experimental data discussed in previous chapters. Quantum mechanical simulations can be seen as a bridge between experiment and theory, especially those based upon first principles methods, because they not only help to explain experimental results, but can also predict properties of new materials or new structures with a great degree of accuracy. A comprehensive analysis on the computational aspects of DFT can be found in "Electronic Structure: Basic Theory and Practical Methods" by Richard M. Martin [68].

To understand the mechanics of DFT, we first step back and look at some elementary quantum mechanics. In quantum mechanics, all the possible information about a system is contained in the system's wave function, $\Psi$. At this point our only focus will be on the electronic structure of atoms, molecules, and solids. The nuclear degrees of freedom are in the form of a potential $v(\mathbf{r})$ that acts on the electrons, and therefore the wave function, depends only on the electronic coordinates. The Hamiltonian for the system of electrons and nuclei is;

$$
H=-\frac{\hbar^{2}}{2 m_{e}} \sum_{i} \nabla_{i}^{2}-\sum_{i, l} \frac{z_{l} e^{2}}{\left|\boldsymbol{r}_{i}-\boldsymbol{R}_{I}\right|}+\frac{1}{2} \sum_{i \neq j} \frac{e^{2}}{\left|\boldsymbol{r}_{i}-\boldsymbol{r}_{j}\right|}-\sum_{l} \frac{\hbar^{2}}{2 M_{I}} \nabla_{I}^{2}+\frac{1}{2} \sum_{I \neq J} \frac{Z_{I} Z_{J} e^{2}}{\left|\boldsymbol{R}_{I}-\boldsymbol{R}_{J}\right|}
$$


where the first term of the equation, $-\frac{\hbar^{2}}{2 m_{e}} \sum_{i} \nabla_{i}^{2}$, is the kinetic energy for the electrons.

The second term, $\sum_{i, l} \frac{z_{l} e^{2}}{\left|\boldsymbol{r}_{i}-\boldsymbol{R}_{I}\right|}$, is the potential acting on the electrons from the nuclei. The third term, $\frac{1}{2} \sum_{i \neq j} \frac{e^{2}}{\left|\boldsymbol{r}_{i}-\boldsymbol{r}_{j}\right|}$, is the electron-electron interaction. The final term,

$\sum_{l} \frac{\hbar^{2}}{2 M_{I}} \nabla_{I}^{2}+\frac{1}{2} \sum_{I \neq J} \frac{Z_{I} Z_{J} e^{2}}{\left|\boldsymbol{R}_{I}-\boldsymbol{R}_{J}\right|}$, is the classical interaction of the nuclei acting with each

other and their kinetic energy. The electrons are denoted by lower case subscripts and the nuclei, with charge $Z_{I}$ and mass $M_{I}$ are denoted by upper case subscripts. This firstprinciples approach gives access to the complete many-electron system. Unfortunately, the number of particles in a solid makes a full quantum mechanical treatment of this system impossible. If we take a first approximation, and regard the $1 / M_{I}$ term in equation 4.1 as very small, by setting the mass of the nuclei to infinity as compared to the electron, then the kinetic energy of the nuclei can be ignored. This is known as the BornOppenheimer approximation, which assumes that the massive nuclei move so much slower than the electron that it can be ignored. The Born-Oppenheimer approximation provides a major simplification to the complicated many-body problem by transforming it into the solution of the electronic dynamics in some frozen configuration of nuclei. This simplifies the Hamiltonian to;

$$
H=\sum_{i=1}^{N}\left(-\frac{\hbar^{2}}{2 m_{e}} \nabla_{i}^{2}-Z e^{2} \sum_{I} \frac{1}{\left|\boldsymbol{r}_{i}-\boldsymbol{R}_{I}\right|}\right)+\frac{1}{2} \sum_{i \neq j} \frac{e^{2}}{\left|\boldsymbol{r}_{i}-\boldsymbol{r}_{j}\right|}
$$


which unfortunately is still impossible to solve because of the large number of electrons. Therefore, we need further approximations to perform efficient and accurate total energy calculations.

The fundamental tenant of density functional theory, proposed in 1964 by Hohenberg and Kohn [69], is that any property of a system of many particles can be viewed as a functional of the ground state density. This means the scalar function of position, in principle, determines all the information in the many-body wave function for the ground and all excited states. A year later Kohn and Sham [70] replaced the difficult interacting many-body system following the Hamiltonian equation 4.1 with a different auxiliary system that can be solved more easily. The Kohn-Sham ansatz is that the ground state density of the original interacting many-body system is equal to that of some chosen non-interacting system. This leads us to independent particle equations for the non-interacting system that are exactly soluble by incorporating all the many-body terms into an exchange-correlation functional of the density. By solving the equations, we find the ground state density and energy of the original interacting system with an accuracy limited only by the approximations in the exchange-correlation functional.

The success of practical, approximate exchange-correlation functionals makes DFT the most widely used method for electronic structure calculations. The crucial quantity in the Kohn-Sham approach is the exchange-correlation energy which is expressed as a functional of the density. The quantum mechanical exchange correlation energy, which contains the many-body contributions, is a small fraction of the total energy, and although not known exactly, it can be approximated very accurately. One of 
the most widely used and simple functionals is the local density approximation (LDA). Kohn and Sham pointed out that solids can often be considered a homogenous electron gas where the nuclei are replaced by a uniform positively charged background, which has the same total density as the overall density of the original matter. The LDA is where the exchange-correlation energy is simply an integral over all space with the exchangecorrelation energy density at each point assumed to be the same as the homogenous electron gas with that density. LDA was further expanded upon and led to the development of the Generalized-gradient approximation (GGA). The GGA includes a functional of the magnitude of the gradient of the electron density and tends to improve total energies, atomization energies, energy barriers and structural energy differences [71].

The Kohn-Sham equations provide the framework for finding the exact density and energy of the ground state of a many-body electron problem using standard independent particle methods. These equations are a set of Schrödinger like independent particle equations which must be solved subject to the condition that the effective potential and density are self-consistent. Solving the Kohn-Sham equations is an iterative process in which the given input potential is used to determine an output density. Only at the exact solution do the input and output densities and potentials agree. The cycle is stopped when some predetermined convergence criterion is reached. A schematic representation of the self-consistent loop for solution to the Kohn-Sham equations can be seen in figure 4.1. Despite the simplification of the original many-body problem to a single particle problem, two difficulties remain for practical and efficient computations to 
be performed; a wavefunction must be calculated for each of the $\sim 10^{23}$ electrons in the system, and the correct description of the electronic wavefunctions, that extend over the entire solid, requires and infinite basis set.

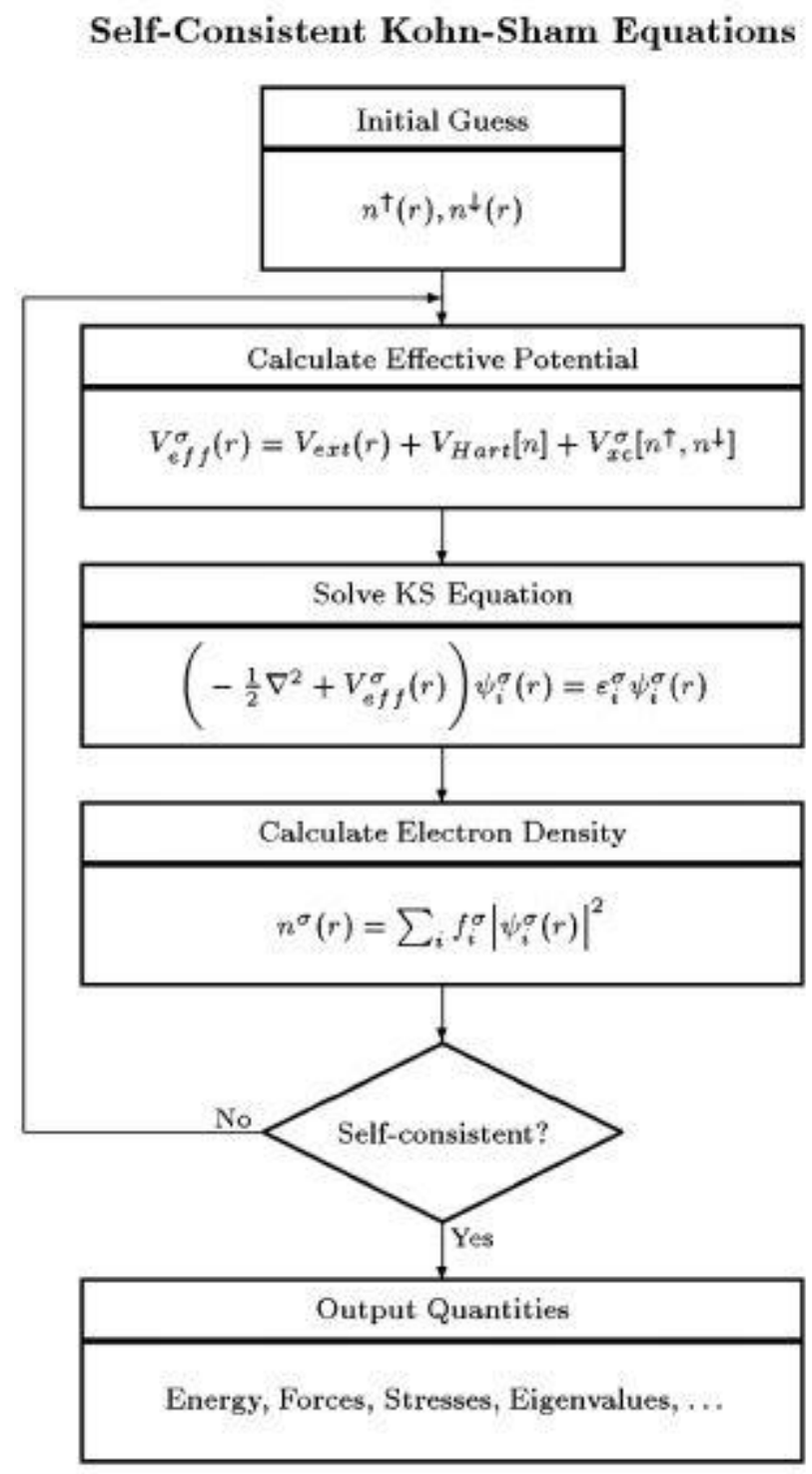

Figure 4.1: Schematic representation of the self-consistent loop of the Kohn-Sham equations [68] 
Since the TMDs are a crystalline solid, they are an ordered state of matter where the positions of the nuclei are repeated periodically in space. The crystal is specified by the types and repetitions of the nuclei in one repeated unit called the primitive unit cell. The position and types of atoms in the primitive cell are called the basis and this set of translations that generate the periodic crystal by repeating the basis is a lattice of points in space called the Bravais lattice. These set of translations forms a group called the space group. Due to the periodicity of the crystal structure, a periodic potential exists and enters into the Schrödinger equation for independent particles as;

$$
H \psi(\boldsymbol{r})=\left[-\frac{\hbar^{2}}{2 m_{e}} \nabla^{2}+V(\boldsymbol{r})\right] \psi_{i}(\boldsymbol{r})=\varepsilon_{i} \psi_{i}(\boldsymbol{r})
$$

where the electrons are no longer free electrons and $V(\boldsymbol{r})$ has the periodicity of the crystal. This leads us to the Bloch theorem, which states that the eigenstates $\psi$ of the Hamiltonian $H$ can be chosen to have the form of a plane wave $\psi_{k}(\boldsymbol{r})=e^{i k \cdot r} u_{k}(\boldsymbol{r})$, where $\boldsymbol{k}$ is a vector in the first Brillouin zone and $u_{k}(\boldsymbol{r})$ is periodic. Electronic states are only allowed at these $k$ points imposed by the boundary conditions. The density of these $k$ points is proportional to the volume of the solid, but only a finite number of electronic states are occupied at each $k$ point. Bloch's theorem has converted an infinite number of electronic wavefunctions of an extended system into a finite number of electronic wavefunctions at an infinite set of $k$ points.

\subsection{Quantum ESPRESSO}


Density functional theory (DFT) provides a powerful tool for the computational analysis of the quantum state of atoms, molecules and solids, and of ab initio molecular dynamics. Quantum ESPRESSO (open Source Package for Research in Electronic Structure, Simulation and Optimization) [72] is an integrated suite of computer codes used for the electronic structure calculations and materials modeling based upon density functional theory, plane wave basis sets and pseudopotentials to represent electron-ion interactions. This research characterized the electronic properties of $\mathrm{MoS}_{2}$ and $\mathrm{WSe}_{2}$ using the Quantum ESPRESSO package in the framework of density functional theory. Ultrasoft pseudopotentials as well as the generalized gradient approximation exchangecorrelation functionals were used in all calculations.

\subsection{Electronic Structure of $\mathrm{MoS}_{2}$}

$\mathrm{MoS}_{2}$ is a layered 2D material of a class of materials called transition metal chalcogenides (or dichalcogenides since there are two chalcogen atoms). The structure consists of two hexagonal planes of the chalcogen atoms, sulfur, separated by the metal atom molybdenum. The sulfur and molybdenum atoms are covalently bonded, while the S-Mo-S units are stacked on top of each other and held together by weak van der Waals forces. $\mathrm{MoS}_{2}$ exists predominately as three polytypes (or stacking arrangements), 1T, $2 \mathrm{H}$, and $3 R$. The $1 T$ phase is unstable and therefore the least common. The $2 \mathrm{H}$ and $3 R$ phases are naturally occurring and identical in structure, with the difference being the stacking of the monolayers (Fig. 4.2). The $2 \mathrm{H}$ phase has hexagonal symmetry having the two monolayers per unit repeat, while the $3 \mathrm{R}$ phase has rhombohedral symmetry with three monolayers per unit repeat. The $3 \mathrm{R}$ phase can be transformed to $2 \mathrm{H}$ upon heating [73]. 
The $2 \mathrm{H}$ phase is the predominant polytype because it is the most stable in nature. The $\mathrm{MoS}_{2}$ bulk unit cell belongs to the $\mathrm{P}_{3} / \mathrm{mmc}$ space group and the lattice constants are $a=$ $3.16 \AA$ and $c=12.295 \AA$.

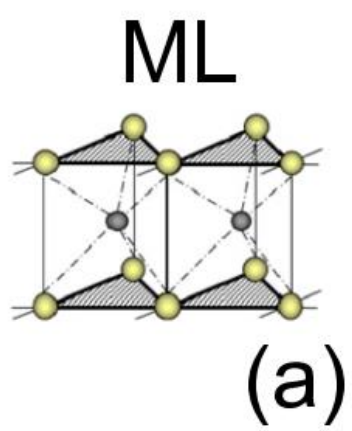

$\circ S$

$\circ$ Mo
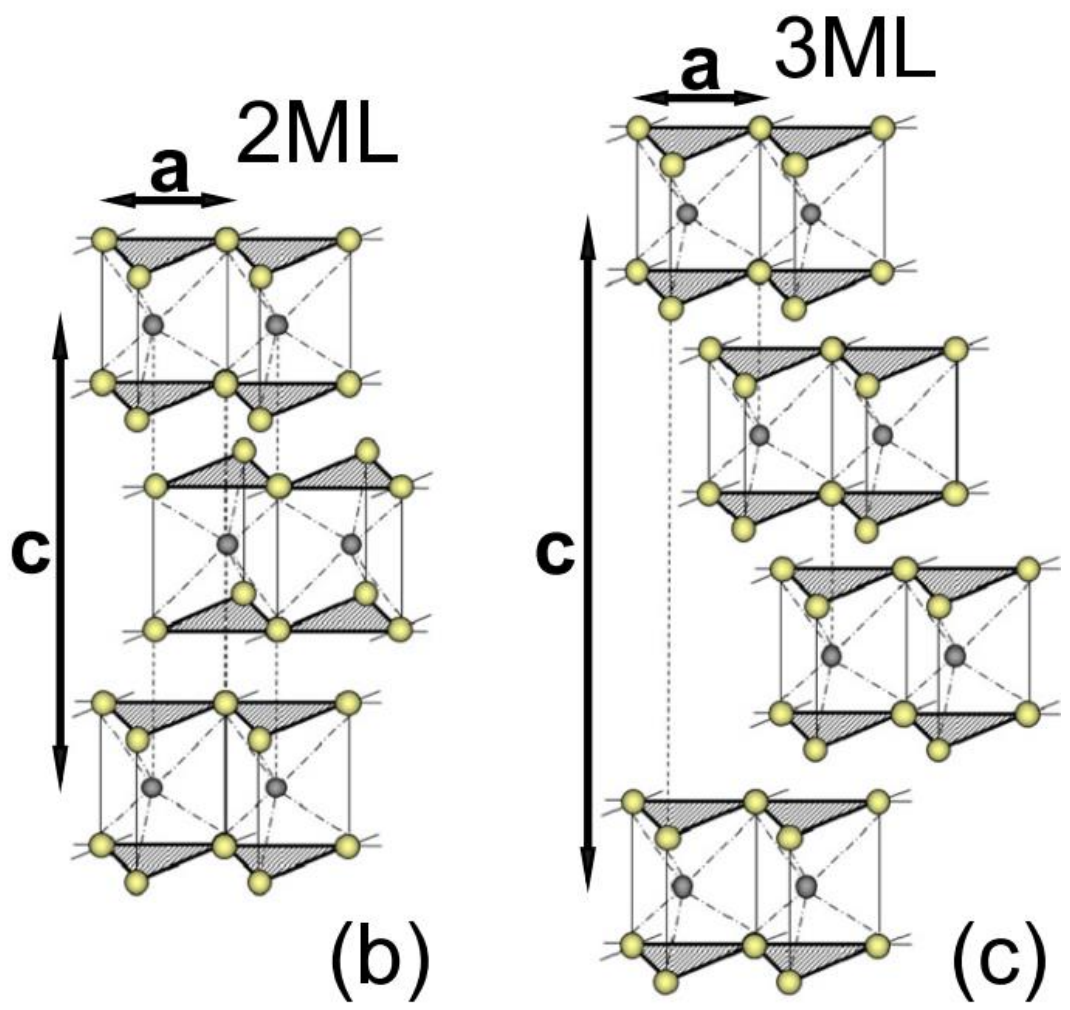

Figure 4.2: (a) Monolayer $\mathrm{MoS}_{2}$ (b) 2 H polytype (c) 3R polytype [74].

The applications of $\mathrm{MoS}_{2}$ in electronic and optoelectronic devices are directly dependent upon its electronic properties such as its electronic band structure and density of states. The band structure of $\mathrm{MoS}_{2}$ plays a critical role in understanding the electron nature and electronic transport in atomically thin films. The band diagram represents the 
energy levels as solutions to the Schrödinger equation for different k-points. The band structure is plotted along the high symmetry direction of the Brillouin zone (BZ). The next few steps obtained by means of the DFT formalism discussed in section 4.1, are presented.

To perform a DFT calculation of periodic structures, we first define the BZ sampling. The Bloch wave vector, $k$, of a given orbital could lie anywhere in the first Brillouin zone. In a general system, there should be, in principle, a set of orbitals for every possible value of $k$. The observables are calculated as an integral over all k-points in the first BZ, but in practice the integration is replaced by a weighted sum and we only deal with a finite set of k-points. These k-points are evenly distributed throughout the BZ according to the Monkhorst-Pack scheme [75]. The symmetry independent volume of the BZ, known as the Irreducible Brillouin Zone (IBZ), will be used.

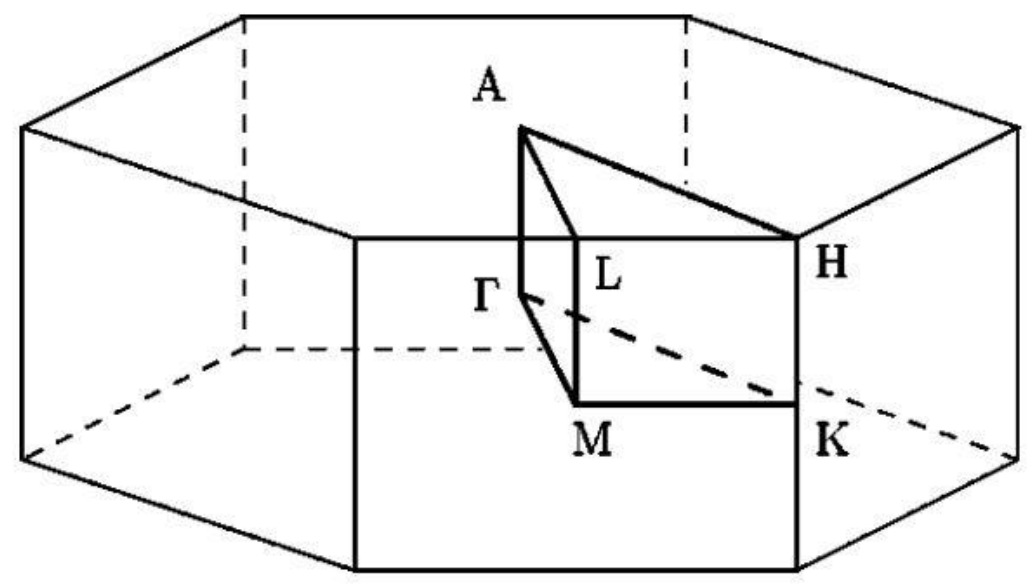

Figure 4.3: The first Brillouin zone of hexagonal unit cell and the IBZ 
The denser the Monkhorst-Pack grid, the more complete and precise the calculated observables will be. Since the electronic wavefunction is represented as the infinite sum of plane waves, the more plane waves, the higher the accuracy, but it comes at a higher computational cost. The plane waves with lower kinetic energy have a higher contribution to the total energy, therefore using the plane waves with the lower energy that still give accurate information is important to find a good balance between accuracy and computational cost. To ensure sufficient sampling, energy convergence studies were performed with respect to the number of k-points and the kinetic energy cutoff value, $\mathrm{E}_{\text {cut. }}$ This data is plotted in the graph of figure 4.4. As can be seen in the graph, increasing the number of k-points above 242 does not lower the total energy significantly, therefore using a 11x11x2 k-point grid yields precise results at a lower computational cost. Increasing $\mathrm{E}_{\text {cut }}$ beyond $40 \mathrm{Ry}(\sim 544 \mathrm{eV})$ does not affect the total energy significantly and therefore the cutoff energy was set to $40 \mathrm{Ry}$. Once the k-point grid and energy cutoff values have been determined, calculations can begin as follows;

1. Self-consistent field (SCF) calculations are performed using Quantum ESPRESSO. The SCF calculations give the self-consistent electron density.

2. After the electron density is obtained, the exchange correlation functionals are obtained and a non-SCF calculation is performed using the full set of k-points in the BZ. 
3. The next step takes the Quantum ESPRESSO results and passes them along to the WanT program for disentangle procedure where the selected number of Wannier functions will be extracted for further localization.

4. WanT then performs the localization and disentanglement procedures for the requested set of Wannier functions.

5. Bands are then calculated by computing the interpolated electronic bandstructure of the system along the selected direction in the BZ.

6. The density of states is then computed interpolating the band-structure by means of the Wannier functions.

The bulk transmittance is calculated from the results obtained in step 4 and is shown later in this section.

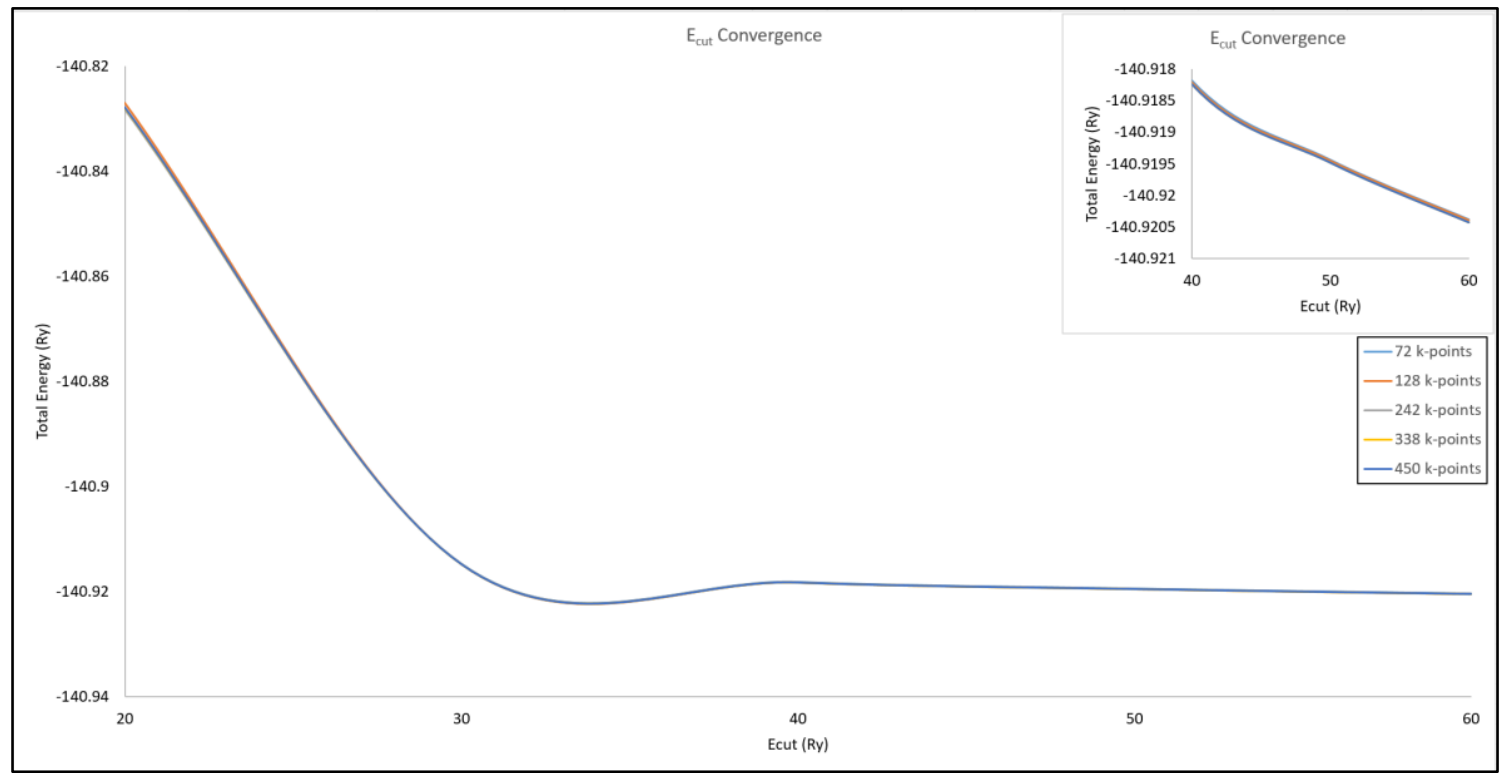

Figure 4.4: Cutoff energy and k-point convergence study. Inset shows small difference with increasing number of k-points. 
Band calculations and orbital projected density of states (PDOS) calculations were made for monolayer, bilayer and bulk thickness of $\mathrm{MoS}_{2}$. The electronic structure band diagram is shown in figure 4.5. The results are very closely matched with those published by several other groups [76-79]. In each band diagram, the red line indicates the bottom of the conduction band and the blue line is the top of the valence band. The difference between the conduction band minimum (CBM) and the valence band maximum (VBM) points indicates the energy band gap. The red arrow indicates the smallest value of the indirect bandgap for bulk and bilayer and the direct bandgap for the monolayer. The bandgaps measured were $\sim 1.3,1.5$, and $1.84 \mathrm{eV}$ for bulk, bilayer and monolayer respectively. These values match closely with those found experimentally [80]. To characterize the bandgap for the bilayer for the film in figure 2.4 , lattice parameter $c$ was set to $13.1 \AA$, consistent with AFM measurement for our film. The bandgap for the bilayer sample shown in figure 2.5 , measured $\sim 1.6 \mathrm{eV}$ with PL, which is slightly higher than the theoretically calculated value of $1.5 \mathrm{eV}$. One possible explanation of this small difference is the underestimation of the bandgap of DFT within LDA or GGA, due to factors such as self-interactions, which is well-known, however the theoretical results are close to our experimental data.

The conduction band states at the K point are mainly due to the localized $d_{x-y}^{2}$ and $d_{x y}$ orbitals of the Mo atoms which are relatively unaffected by the interlayer coupling since they are sandwiched between the $S$ atoms. The states near the $\Gamma$ point are due to combination of the antibonding $p_{z}$ orbitals of the $\mathrm{S}$ atoms and the $d_{z}{ }^{2}$ orbitals of the 
Mo atoms. These have a strong interlayer coupling effect [81], so as the number of layers change, the direct exitonic states near the K point remain relatively unchanged, but the transition at the $\Gamma$ point shifts from indirect to direct as the number of layers reduces to a single layer. The change in band structure with the number of layers is due to quantum confinement and the change in hybridization between the $p_{z}$ orbitals on $\mathrm{S}$ atoms and the $d_{z}^{2}$ orbitals on Mo atoms [33]. Therefore, the absence of the interlayer coupling is the origin of the direct bandgap of single layer $\mathrm{MoS}_{2}$. This direct bandgap transition opens exciting possibilities for optoelectronics. 
bulk

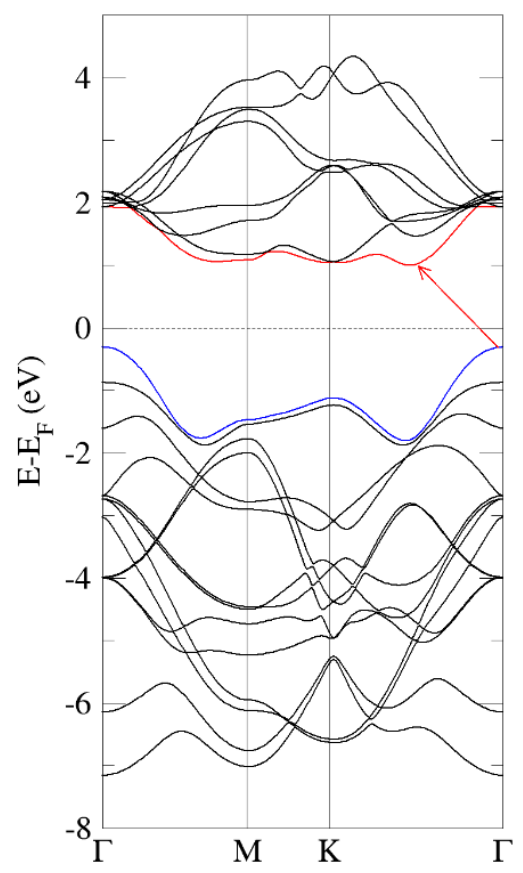

bilayer

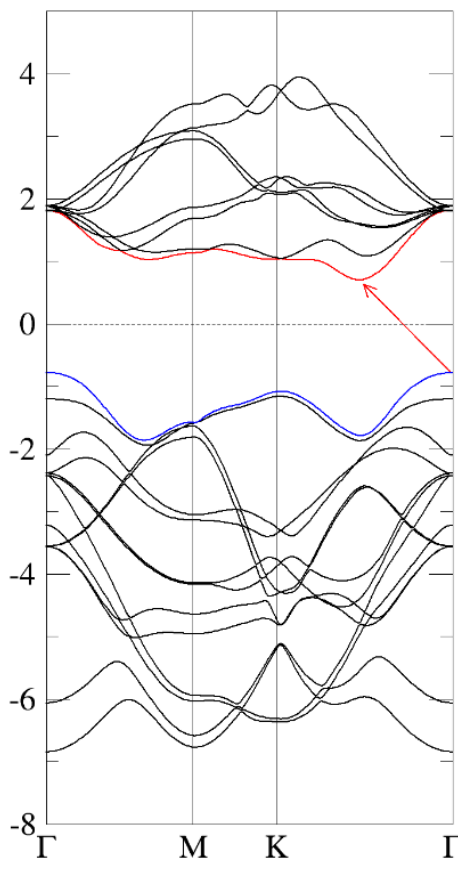

monolayer

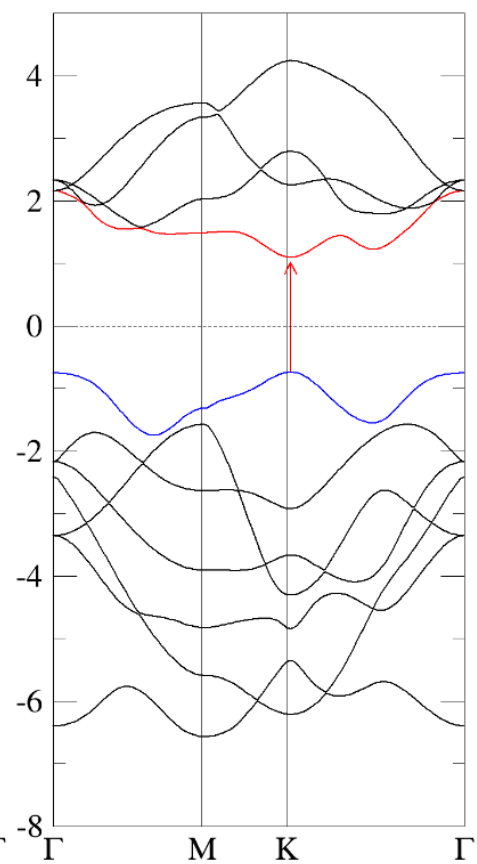

Figure 4.5: Electronic band structure of bulk, bilayer, and monolayer $\mathrm{MoS}_{2}$.

The comparison between bulk, bilayer, and monolayer projected density of states (PDOS) is shown in figure 4.6. From the figure, the electronic states near the Fermi level are mostly due to the out-of-plane $d_{z^{2}}$ and in-plane $d_{x y}$ and $d_{x^{2}-y^{2}}$ states in Mo, while the $p_{z}$ state in $\mathrm{S}$ is predominate. This order of electronic states dominance does not change 
going from bulk to monolayer.

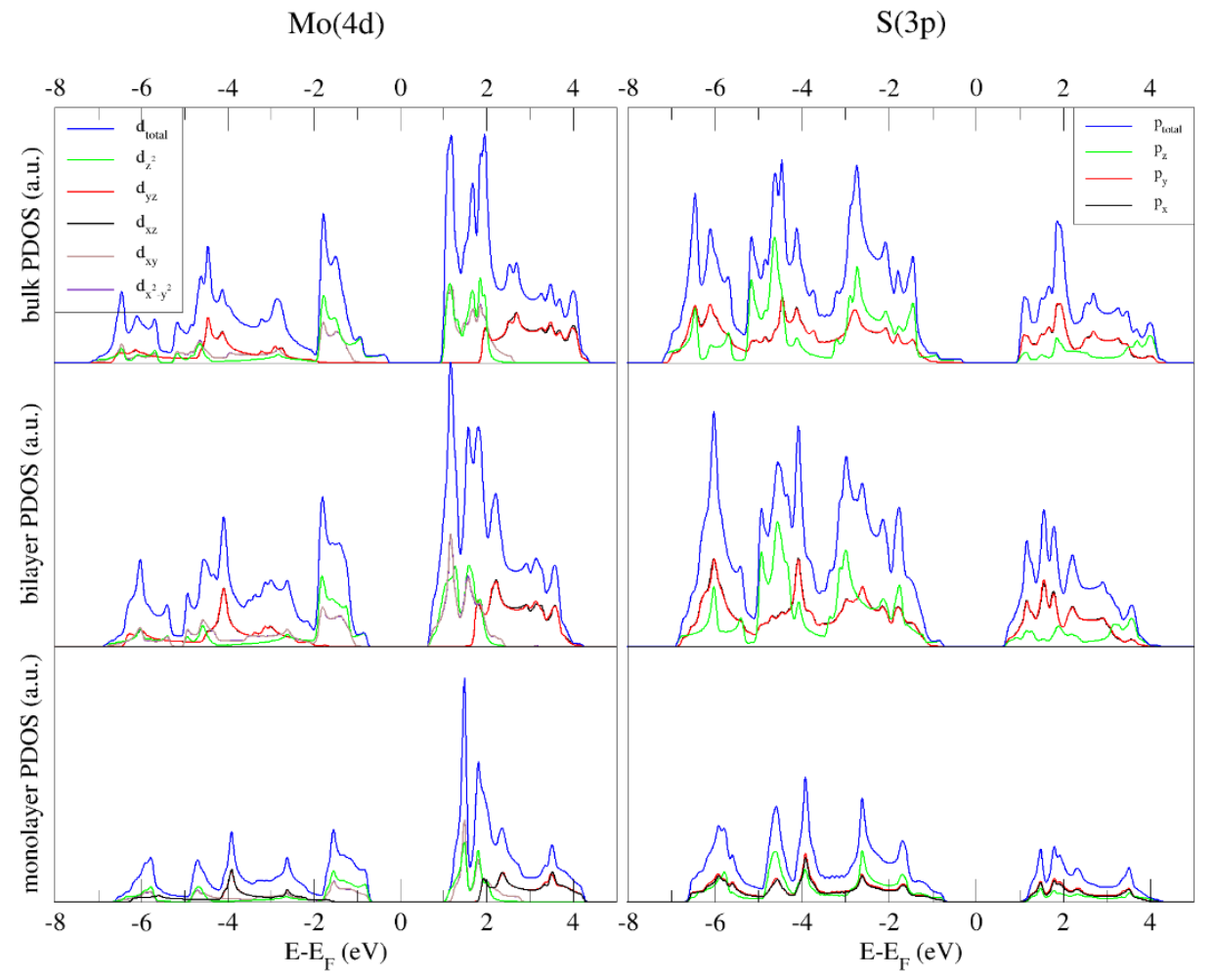

Figure 4.6: Calculated projected density of states (PDOS) of bulk, bilayer, and monolayer $\mathrm{MoS}_{2}$ for $\mathrm{Mo}(4 d)$ and $S(3 p)$ states

The electronic band structure and corresponding density of states of bilayer $\mathrm{MoS}_{2}$ is shown in figure 4.7. Since the sample discussed in chapter 2 is a bilayer $\mathrm{MoS}_{2}$ film, the band structure and density of states for bilayer was investigated. The electronic band structure and density of states can be divided into three sets of bands and states respectively. The first set of bands around $-14 \mathrm{eV}$ is mainly due to the $3 s$ orbital of the $\mathrm{S}$ atom which is separated by a large gap from the second set of bands. The second set of 
bands just below the Fermi energy are mainly due to the $\mathrm{S} 3 p$ and Mo $4 d$ orbitals which shows strong hybridization as previously discussed. The third set of bands just above the Fermi energy has its main contribution from the Mo $4 d$ orbital.

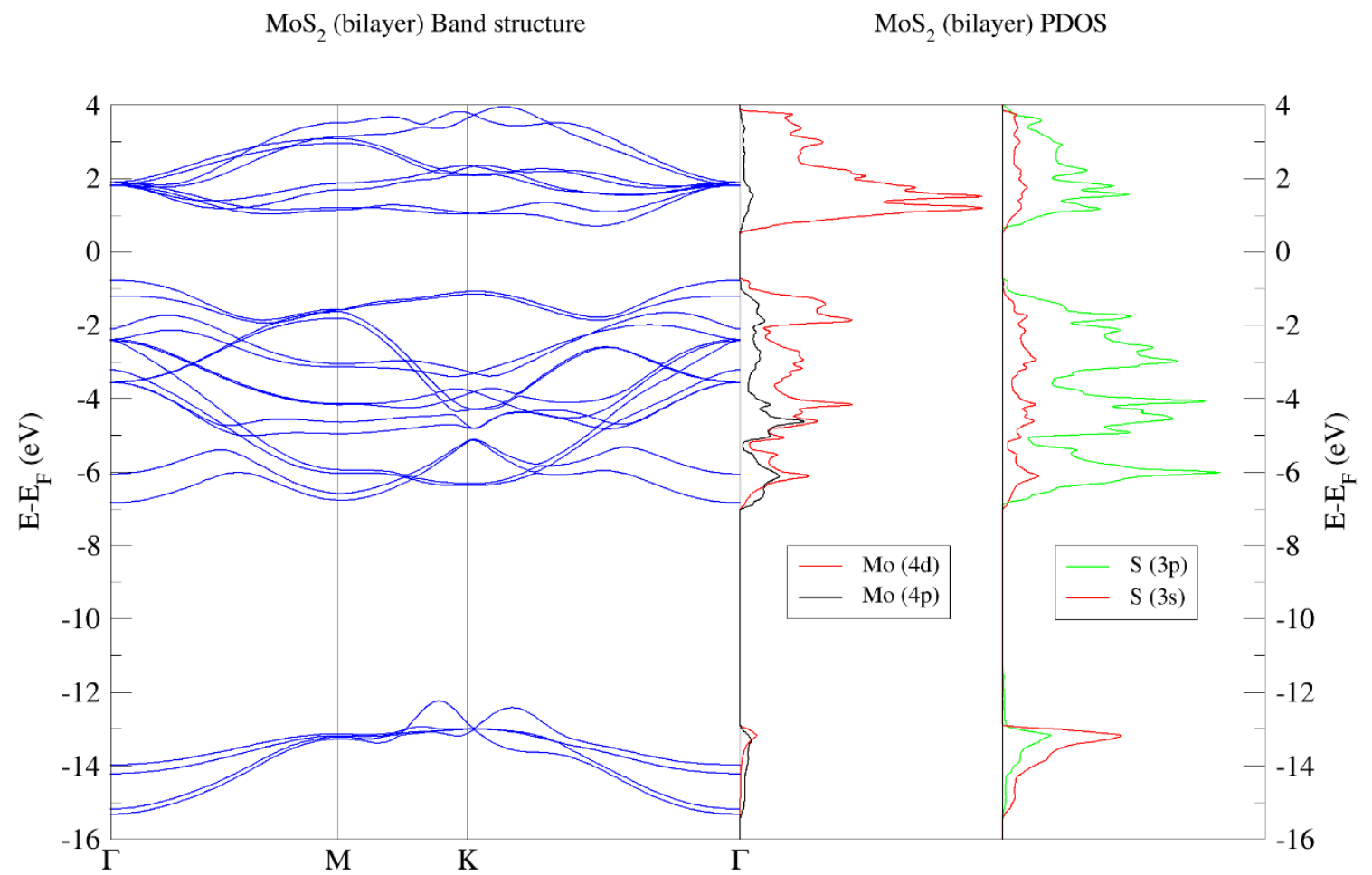

Figure 4.7: Calculated band structure and orbital projected density of states (PDOS) for bilayer $\mathrm{MoS}_{2}$

After completing the band structure and density of states calculations, we further investigate electron transport in a bilayer film. Transmittance calculations were performed as part of the WanT [82-84] code in Quantum ESPRESSO using maximally localized Wannier functions. WanT uses the plane wave ultra-soft pseudopotential 
calculations with a Green's function method that is based upon Landauer formalism to describe quantum conductance. The essential connection between the DFT calculations and the Green's functions is the use of maximally localized Wannier functions to introduce the ground state electronic structure into the lattice Green's function approach at the basis of the evaluation of quantum conductance. Bloch orbitals cannot be used directly to calculate electronic transport because they are delocalized. The Green's function relies on localized orbital representations; therefore, the Bloch orbitals must be transformed into localized functions, known as the Wannier functions. This transformation is described in detail by Mazari and Vanderbilt [84]. Wannier functions are the solid-state equivalent of localized molecular orbitals and they provide a valuable insight to the chemical bonding that is otherwise missing from the Bloch picture of extended orbitals. In essence they are the real-space picture of localized orbitals. The Wannier functions for the Mo $4 d$ and S $3 p$ atoms calculated from the bilayer $\mathrm{MoS}_{2}$ film are plotted in figures 4.8 and 4.9. The isosurface plots shown are consistent with $d$ and $p$ orbitals and provide a qualitative picture of the electronic structure of bilayer $\mathrm{MoS}_{2}$. 

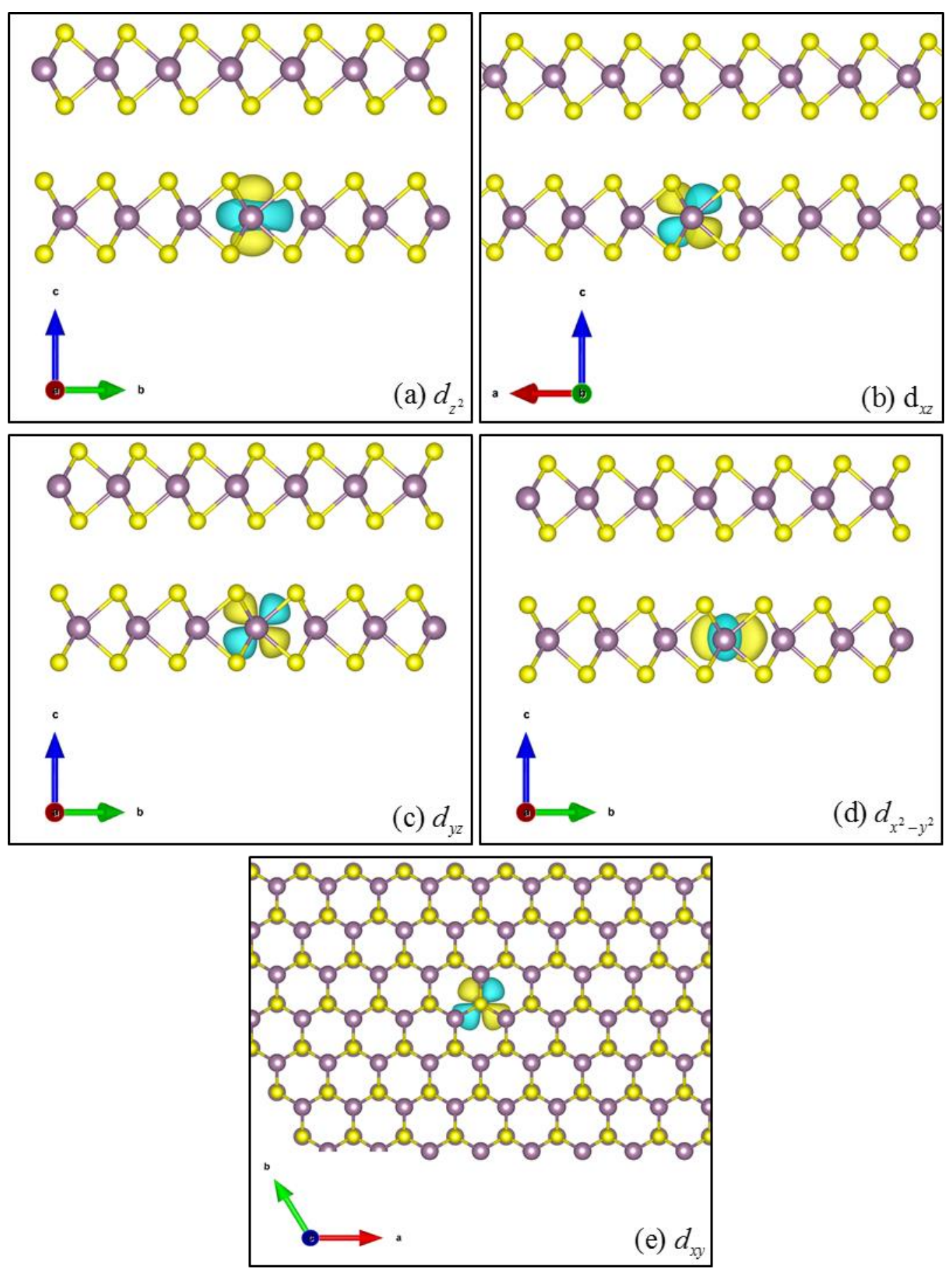

Figure 4.8: Wannier functions of the Mo 4d orbitals for bilayer MoS2. Yellow and bluegreen isosurfaces correspond to positive and negative charge respectively. 


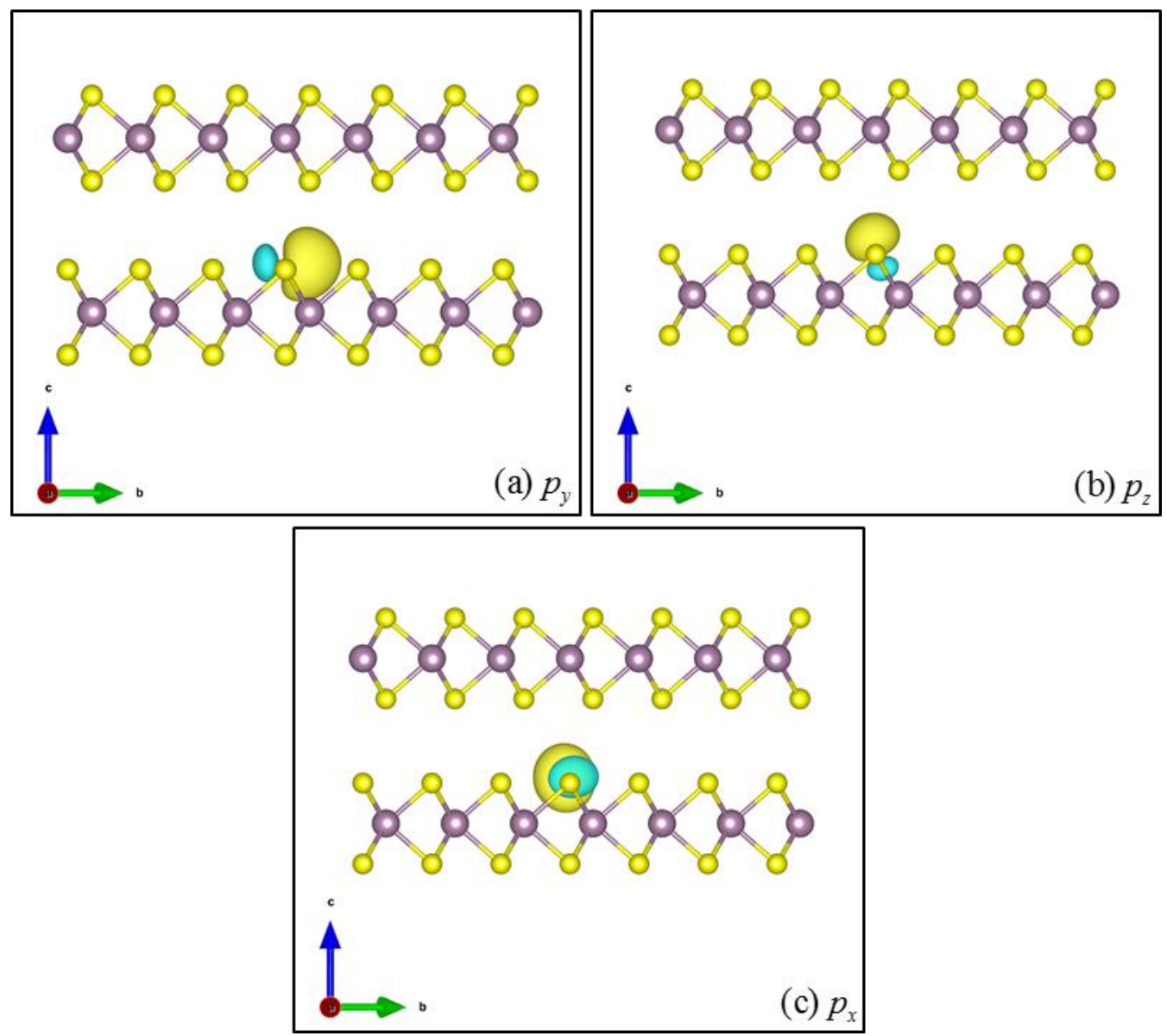

Figure 4.9: Wannier functions of the $S 3 p$ orbitals for bilayer $M o S_{2}$. Yellow and bluegreen isosurfaces correspond to positive and negative charge respectively.

The bulk transmittance calculations were made using the Hamiltonian matrices calculated during the Wannierization process in the previous steps. The main output is the quantum transmittance across the $\mathrm{MoS}_{2}$ bilayer film. The theoretical quantum conductance output is given in units of $\frac{2 e^{2}}{h}$ (or $\left.77.46 \mu \mathrm{S}\right)$ and is plotted in figure 4.10. The complete picture with bands, conductance, and density of states is shown in figure 4.11 and matches previous calculations found in literature [77]. 


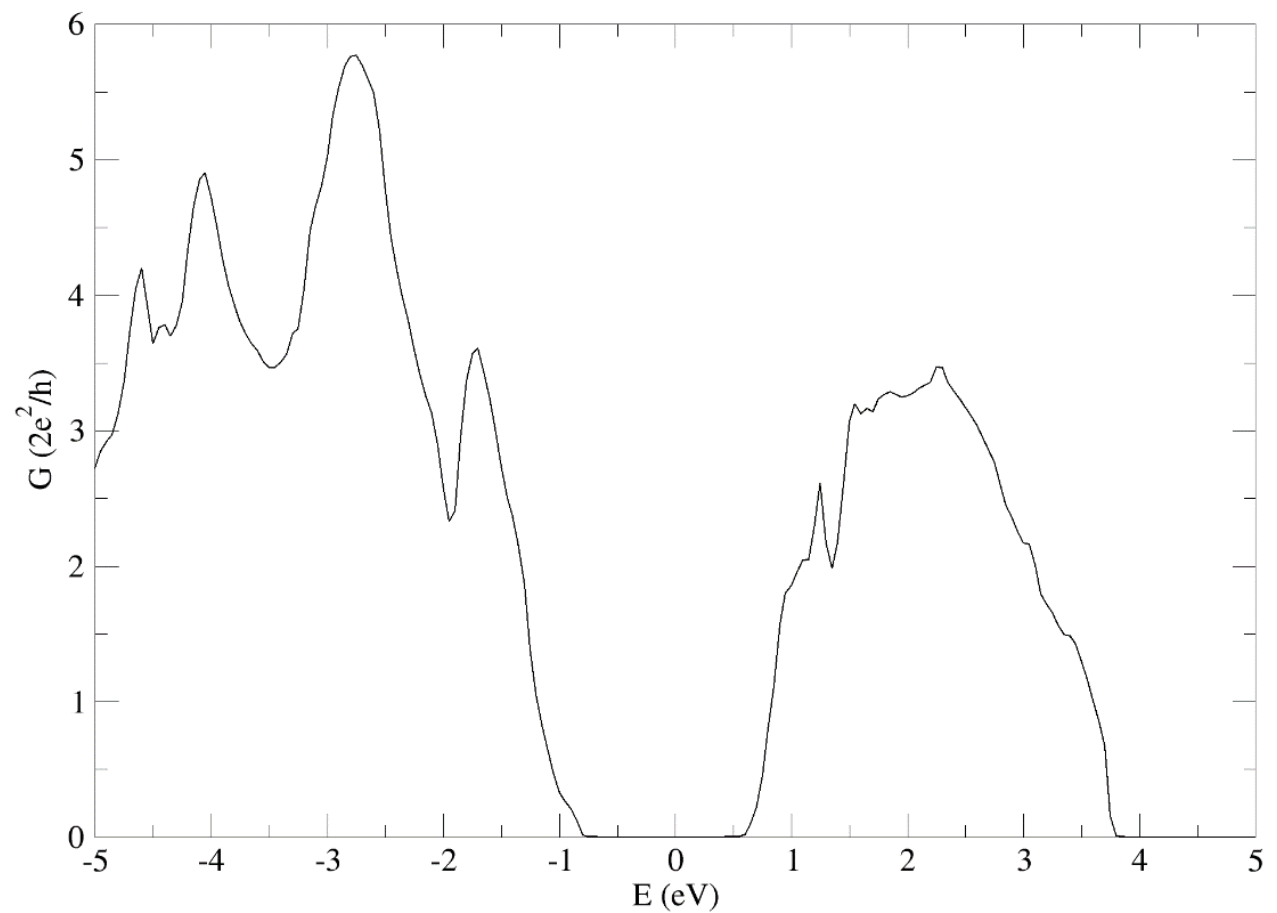

Figure 4.10: $\mathrm{MoS}_{2}$ bilayer film theoretical quantum conductance 

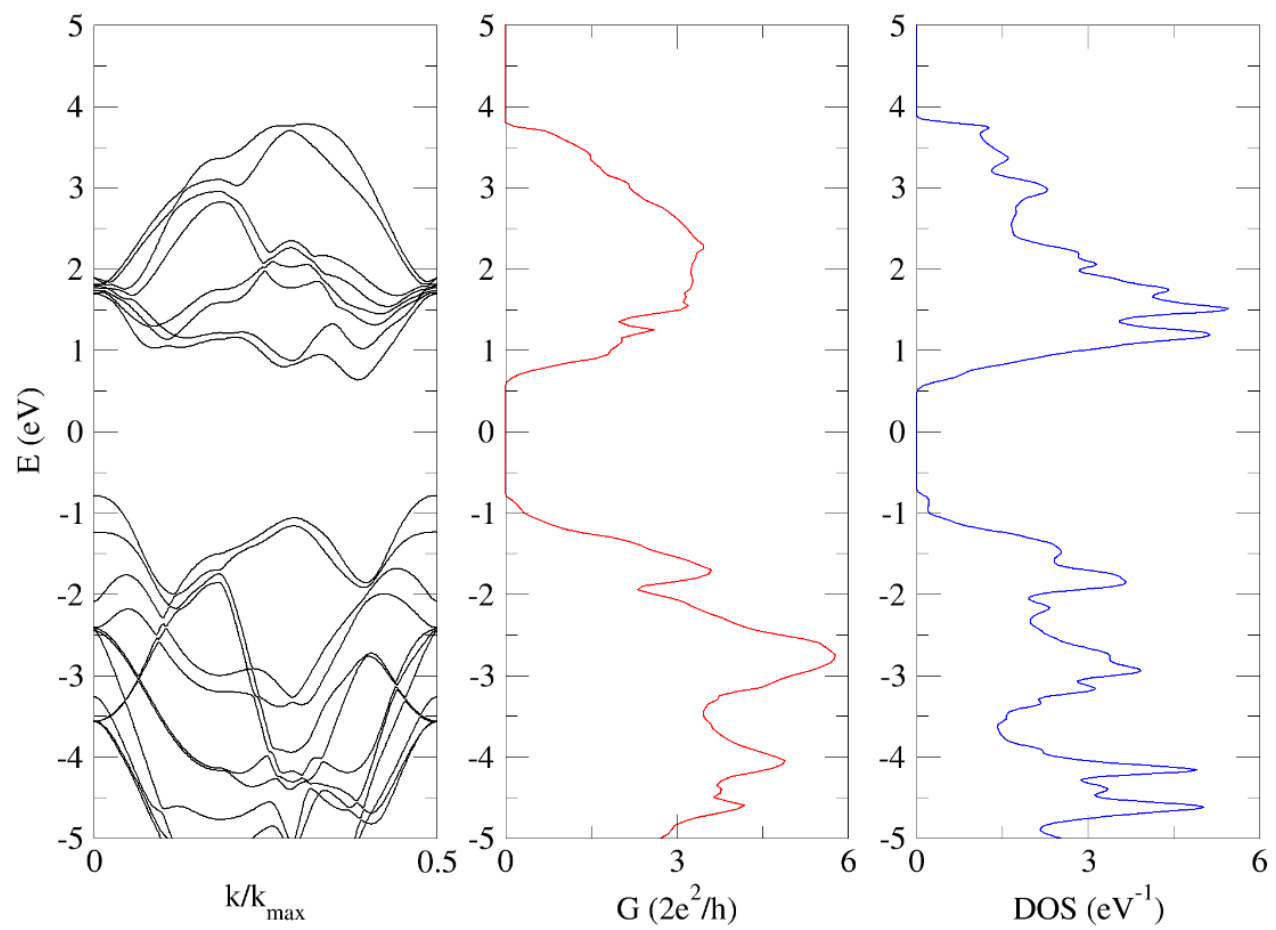

Figure 4.11: $\mathrm{MoS}_{2}$ bilayer band structure, conductance, and density of states

\subsection{Electronic Structure of WSe2}

The electronic structure of $\mathrm{WSe}_{2}$ has many similarities to that of $\mathrm{MoS}_{2}$ and therefore some of the data will be similar. The $\mathrm{WSe}_{2}$ structure consists of two hexagonal planes of the chalcogen atoms, selenium, separated by the metal atom tungsten. The selenium and tungsten atoms are covalently bonded, while the Se-W-Se units are stacked on top of each other and held together by the weak van der Waals forces. WSe $\mathrm{W}_{2}$ exists 
predominately as three polytypes; $1 \mathrm{~T}, 2 \mathrm{H}$, and $3 \mathrm{R}$, the same as $\mathrm{MoS}_{2}$. The $1 \mathrm{~T}$ phase is unstable and therefore the least common. The $2 \mathrm{H}$ and $3 \mathrm{R}$ phases are identical in structure with the difference being the stacking of the monolayers (see $\mathrm{MoS}_{2}$ example in Fig. 4.2). The $2 \mathrm{H}$ phase has hexagonal symmetry with $\mathrm{W}$ atoms in a prismatic arrangement with the Se atoms, and the two monolayers per unit repeat, while the $3 \mathrm{R}$ phase has rhombohedral symmetry with three monolayers per unit repeat. The $2 \mathrm{H}$ phase is the most predominant polytype. The $\mathrm{WSe}_{2}$ bulk unit cell belongs to the $\mathrm{P}_{3} / \mathrm{mmc}$ space group. The $\mathrm{W}$ and Se atoms are larger than the Mo and S atoms, therefore the lattice constants are slightly larger with $a=3.282 \AA$ and $c=12.96 \AA$. The electronic configuration of the $\mathrm{W}$ atoms is $[\mathrm{Xe}] 4 f^{14} 5 d^{4} 6 s^{2}$ and $\mathrm{Se}$ is $[\mathrm{Ar}] 3 d^{10} 4 s^{2} 4 p^{4}$. This causes a strong $p-d$ interaction between the $\mathrm{W}$ and Se layers within each monolayer, while the interaction between the layers is only the weak van der Waals force.

Prior to performing the band structure calculations, we had to determine the proper density of the Monkhorst-Pack grid that was used for calculating the wavefunctions. Following a similar methodology to that used for $\mathrm{MoS}_{2}$, energy convergence studies were performed with respect to the number of k-points and the kinetic energy cutoff value, $\mathrm{E}_{\mathrm{cut}}$. This data is plotted in the two graphs shown in figure 4.12. As can be seen in the graph 4.12(a), increasing the number of k-points above 288 does not lower the total energy significantly, therefore using a 12x12x2 k-point grid yields precise results with a lower computational cost. The energy convergence study for $\mathrm{E}_{\text {cut }}$ in the graph 4.12 (b) shows that beyond $40 \mathrm{Ry}(\sim 544 \mathrm{eV})$ the total energy is not significantly reduced, therefore the cutoff energy was set to $40 \mathrm{Ry}$. Once the k-point grid 
and energy cutoff values were determined, calculations were performed using the same procedure as in section 4.3 .
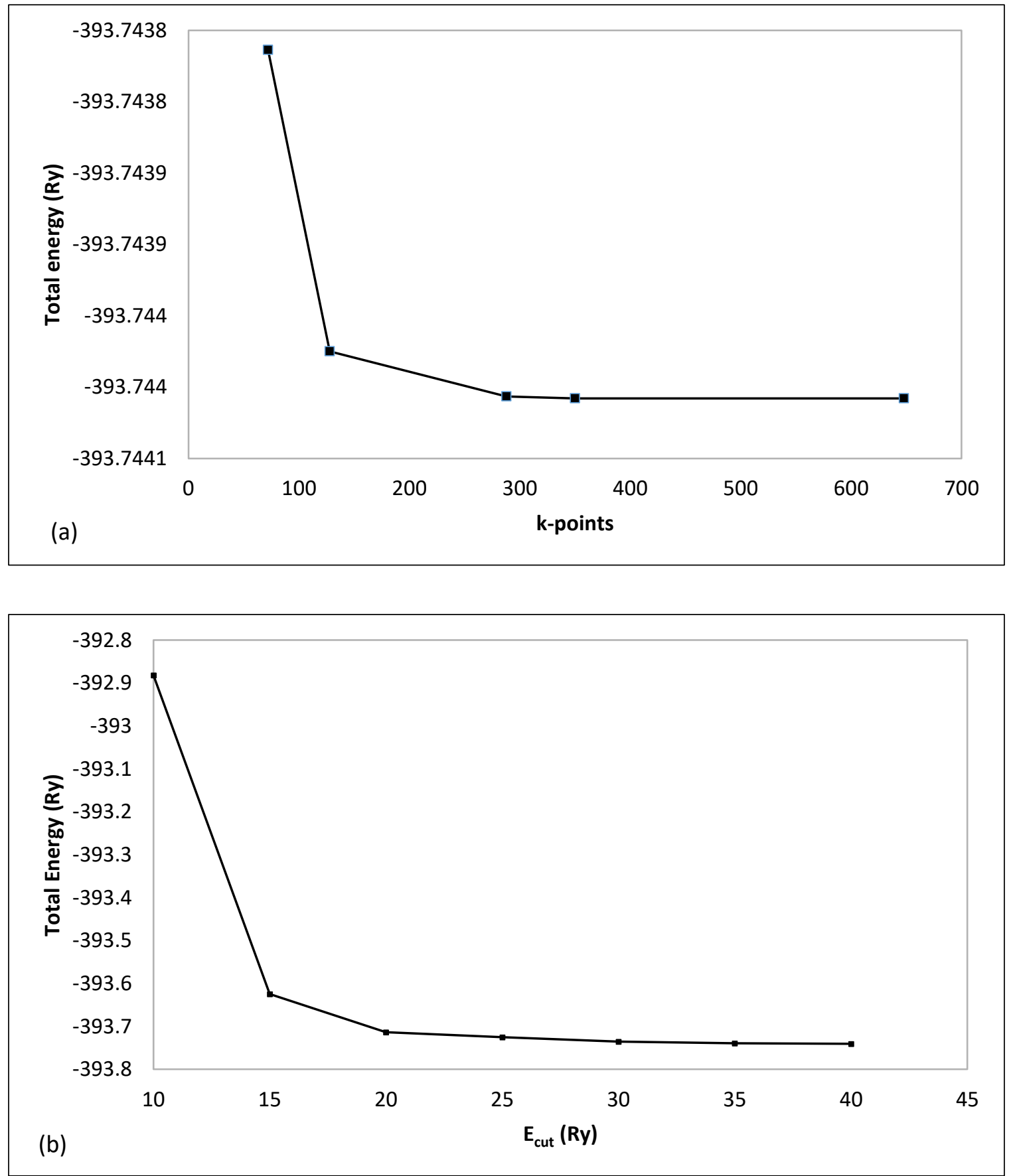

Figure 4.12: (a) k-point and (b) $E_{\text {cut }}$ convergence study 
Band calculations and orbital projected density of states (PDOS) calculations were made for monolayer and bulk WSe 2 . Since no film thicknesses fewer than about four layers were grown, only monolayer and bulk were compared so that the difference between bulk and monolayer can be shown. $\mathrm{WSe}_{2}$ is very similar to $\mathrm{MoS}_{2}$ in its band diagram (Fig. 4.13) in that it is a direct band gap semiconductor at the monolayer with the gap occurring at the $\mathrm{K}$ point. One key difference between $\mathrm{MoS}_{2}$ and $\mathrm{WSe}_{2}$ is the size of the spin orbit splitting due to the different size of the transition metal. $\mathrm{WSe}_{2}$ has a larger spin orbit coupling with the splitting of the VBM (valence band maximum) $\sim 0.46 \mathrm{eV}$ in monolayer while $\mathrm{MoS}_{2}$ spin orbit splitting is $\sim 0.12 \mathrm{eV}$ in the monolayer. The spin orbit physics of these materials are interesting because of the potential application in spintronics and optoelectronics, but is not investigated here. In bulk $\mathrm{WSe}_{2}$ the indirect gap occurs between the $\Gamma$ and $\mathrm{K}$ point on the $\mathrm{CBM}$ and the $\Gamma$ point at the VBM. The red arrow between the CBM and VBM in figure 4.13 indicates the band gap. In the case of the bulk $\mathrm{WSe}_{2}$ film the theoretical band gap is indirect at $\sim 1.15 \mathrm{eV}$ while the monolayer band gap is $\sim 1.65 \mathrm{eV}$, which is consistent with previously reported values $[65,79,85]$. For $\mathrm{WSe}_{2}$ the transition from direct to indirect band gap differs by $\sim 40 \mathrm{meV}(\sim 500 \mathrm{meV}$ from monolayer to bulk) while for $\mathrm{MoS}_{2}$ that difference is $\sim 360 \mathrm{meV}(\sim 700 \mathrm{meV}$ from monolayer to bulk). 

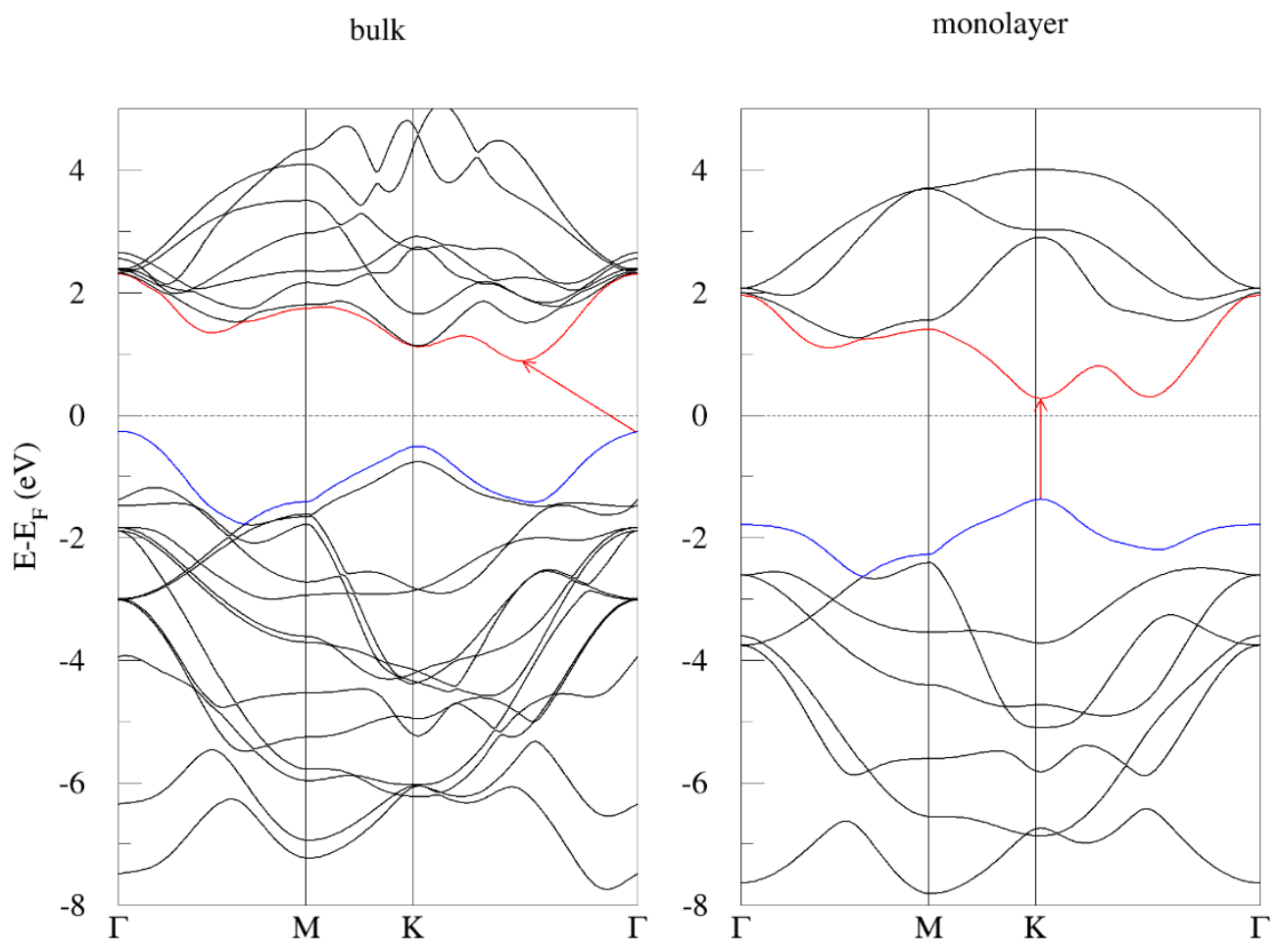

Figure 4.13: Electronic band structure of bulk and monolayer $\mathrm{WSe}_{2}$

The comparison between bulk and monolayer projected density of states (PDOS) is shown in figure 4.14. From the figure, the electronic states near the Fermi level are observed to be mostly due to the out-of-plane $d_{z^{2}}$ and in-plane $d_{x y}$ and $d_{x^{2}-y^{2}}$ states in W 
at the conduction bands, while the $p_{z}$ state in Se at the valence bands is predominant.

W $(4 d)$

$\operatorname{Se}(3 p)$

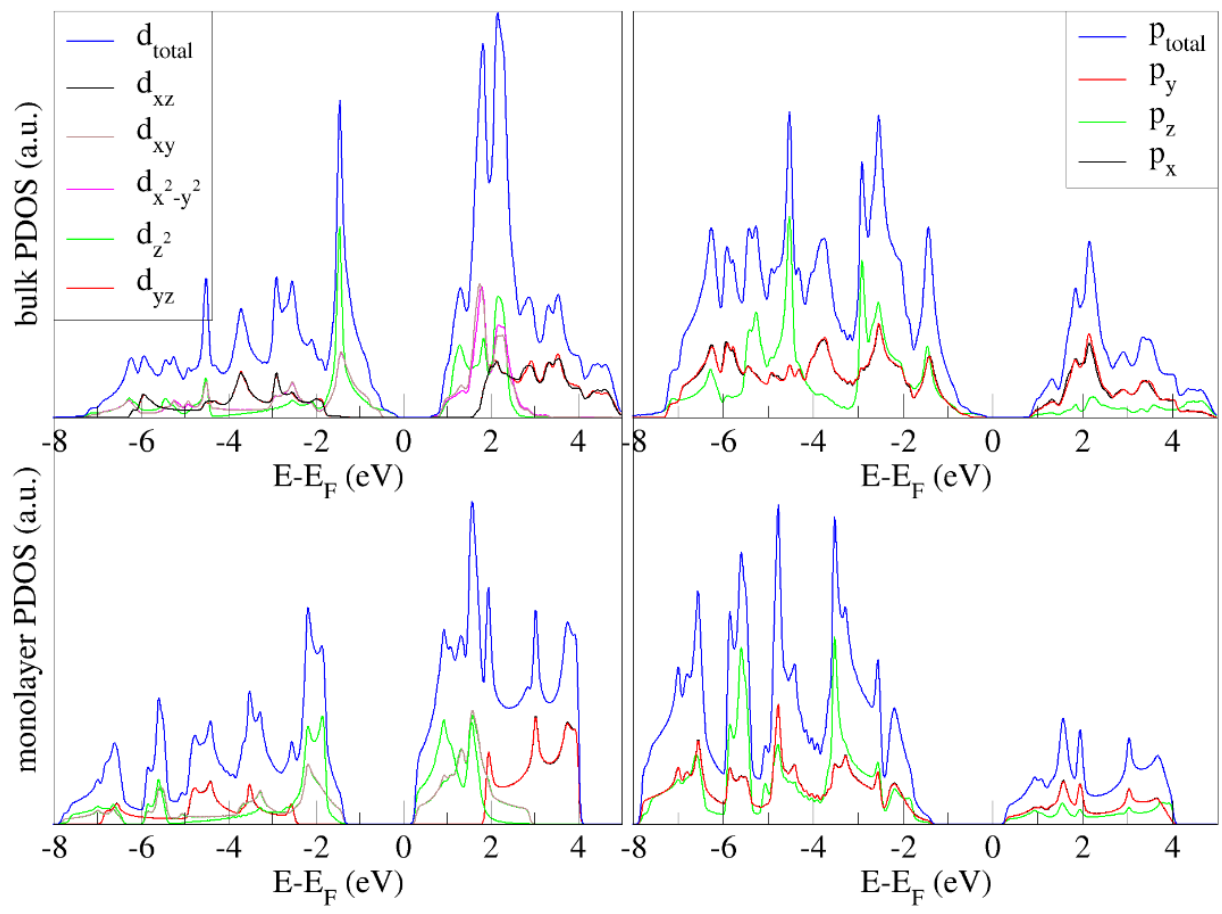

Figure 4.14: Calculated projected density of states (PDOS) of bulk and monolayer WSe 2 for $W(4 d)$ and $\mathrm{Se}(3 p)$ states

A side by side comparison of the electronic band structure and the orbital projected density of states (Fig. 4.15) shows that the states at the top of the valence band are a result of the Se $p$-orbitals, while the bottom of the conduction band is due to the $\mathrm{W}$ 
$d$-orbitals.

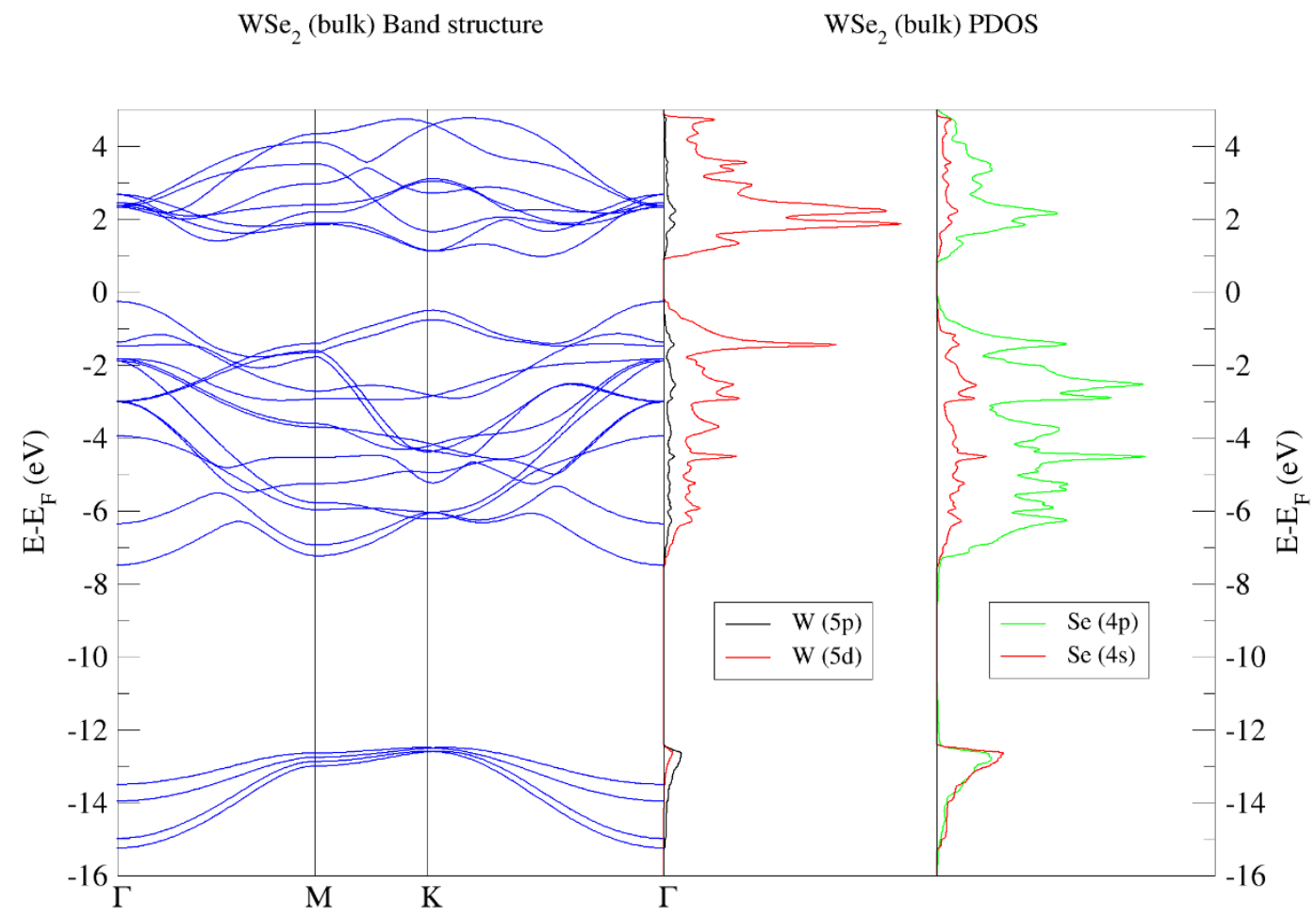

Figure 4.15: Calculated band structure and orbital projected density of states (PDOS) for bulk $\mathrm{WSe}_{2}$

Transmittance calculations were performed for bulk $\mathrm{WSe}_{2}$ as part of the WanT code in Quantum ESPRESSO using maximally localized Wannier functions, following the same procedures as described in section 4.3.1 for $\mathrm{MoS}_{2}$. The Wannier functions for the W $5 d$ and Se $4 p$ atoms calculated from the bulk WSe 2 film are plotted in figures 4.16 and 4.17. The spherical symmetry is evident and the isosurface plots are consistent with $d$ 
and $p$ orbitals and provide a qualitative picture of the electronic structure of bulk $\mathrm{WSe}_{2}$. 

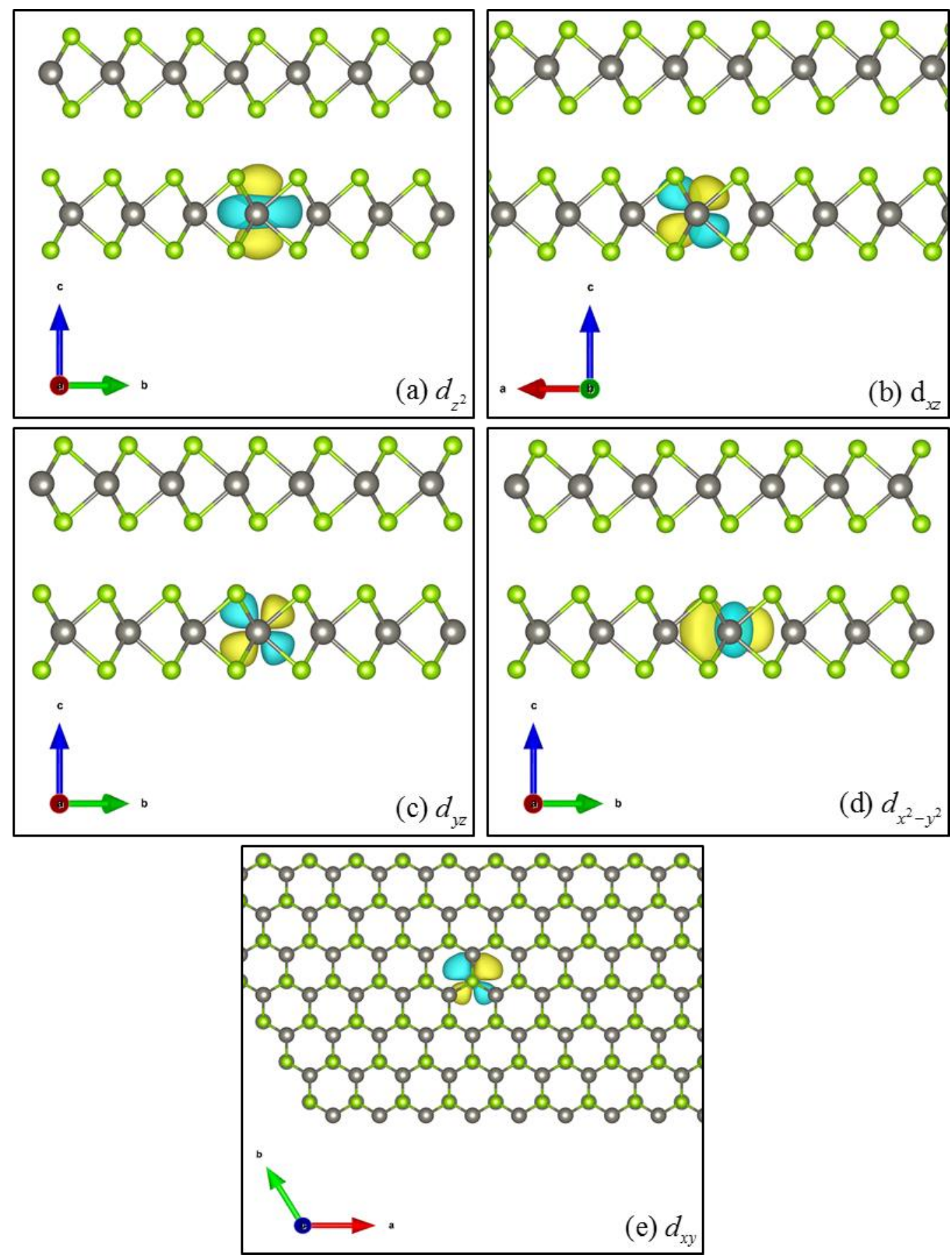

Figure 4.16: Wannier functions for the 5d orbitals for bulk WSe2. Yellow and blue-green isosurfaces correspond to positive and negative charge respectively. 


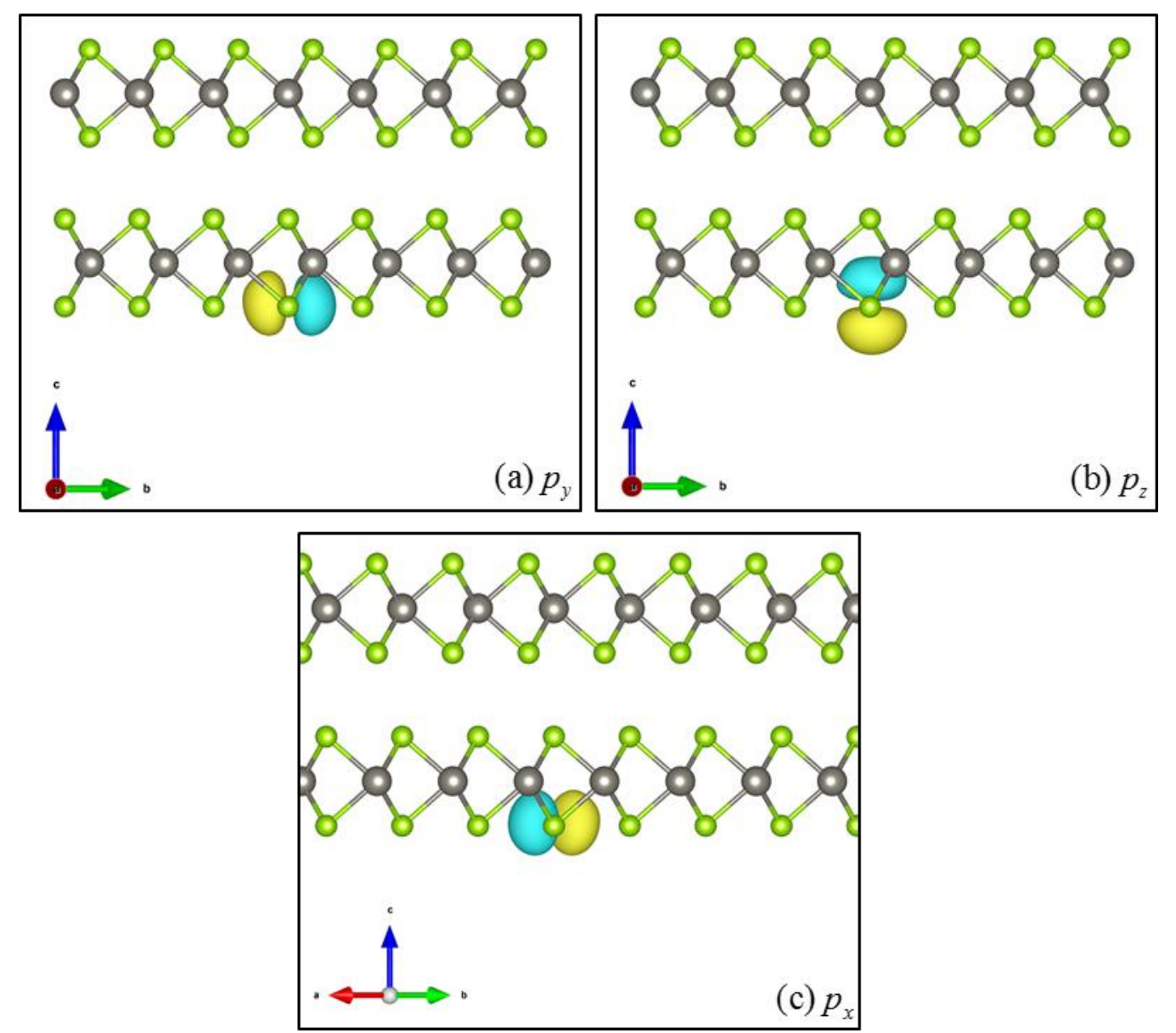

Figure 4.17: Wannier functions for the 4p orbitals for bulk WSe. Yellow and blue-green isosurfaces correspond to positive and negative charge respectively.

The theoretical bulk transmittance calculations were completed for $\mathrm{WSe}_{2}$ and compared to the experimental conductance data calculated from the electrical data in figure 3.14 from our bulk $\mathrm{WSe}_{2}$ film. The theoretical conductance (transconductance or $\left.\mathrm{g}_{\mathrm{m}}\right), g_{m}=\left.\frac{d I_{d}}{d V_{G S}}\right|_{V_{D S}}$, plot profile matched the experimental conductance plot very well, but is higher by a factor of about 100 (Fig. 4.18) because the experimental plot contains 104 
resistance due to the metal contacts. The slight shift to the right of zero bias voltage is due to the p-type nature of the $\mathrm{WSe}_{2}$ film.

Theoretical

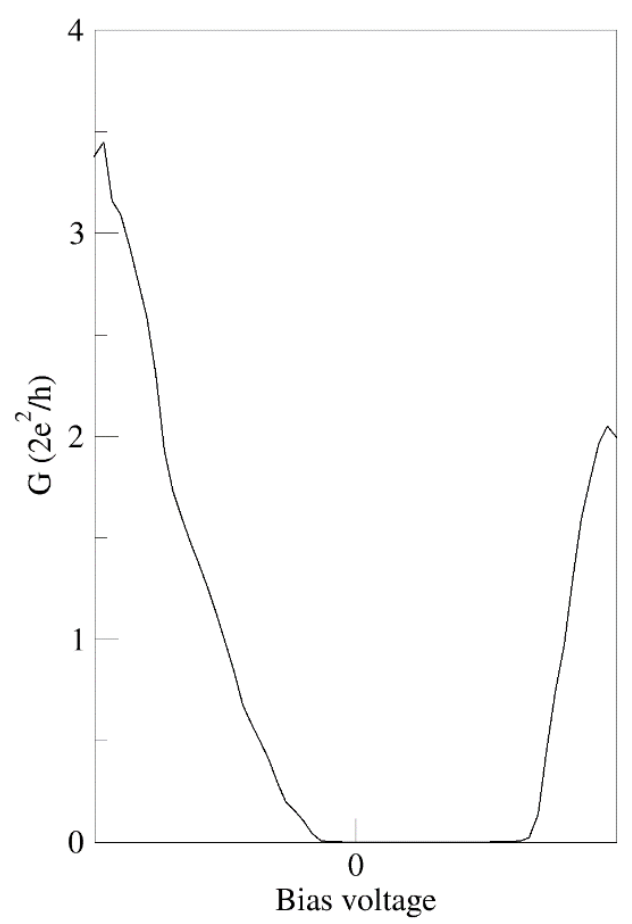

Experimental

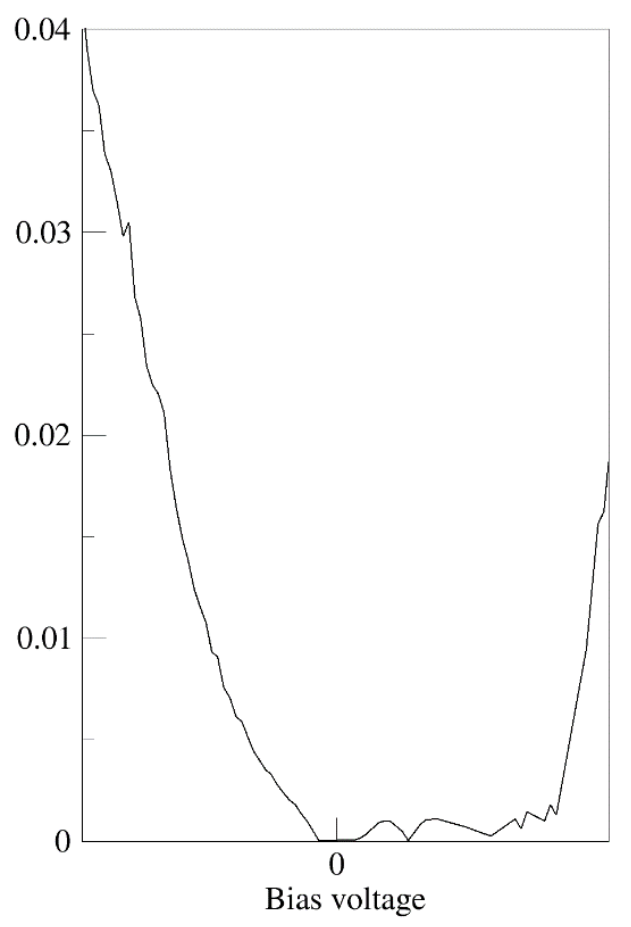

Figure 4.18: Comparison of experimental and theoretical conductance of bulk $\mathrm{WSe}_{2}$ film.

To compare the experimental data with the theoretical data, the theoretical conductance

( $G$ ) was converted to resistivity with the following formula: $\rho=S \times \frac{12900 \Omega}{G}$, where $12900 \Omega$ is the quantum conductance and $S$ is the shape factor. The shape factor is added because the calculations were made on a $6 \times 6$ hexagonal supercell and resistivity is unit 
area, therefore, $S=6 \times 6 \times \sqrt{3}$. The $\sqrt{3}$ factor is from the conversion of the hexagonal cell metrics into a rectangular structure. The contact resistances, $R_{c}$, for these devices were calculated for $\mathrm{Pd}$ and $\mathrm{Ni}$ contacts in section 3.3. The experimental contact resistance was added to the total resistance of the theoretical data and the theoretical conductance was recalculated and overlaid with the experimental conductance in the graphs shown in figures 4.19 and 4.20. For the device with Pd contacts, the theoretical and experimental curves fit very well. The experimental conductance for hole injection (left side of the curve) starts to increase just to the left of $0 \mathrm{~V}$ gate bias at about $-0.3 \mathrm{~V}$. The electron injection side (right side of curve) of the curve has a sudden increase in conductance at about $1 \mathrm{~V}$. The fermi level of $\mathrm{Pd}$ is closer to the valence band of $\mathrm{WSe}_{2}$, reducing the height of the Schottky barrier and allowing for better hole injection, while the Schottky barrier height for electron injection into the conduction band is higher [63]. The theoretical vs. experimental conductance plot using Ni contacts is shown in the graph of figure 4.20. The contact resistance value calculated from the $\mathrm{WSe}_{2} \mathrm{FET}$ with Ni contacts, was added to the total resistance of the theoretical data as described above. The experimental conductance matches well with the theoretical for the device with $\mathrm{Ni}$ contacts. The experimental conductance on the left side of the curve (hole injection) for the device with $\mathrm{Ni}$ contacts starts to increase at about $-0.4 \mathrm{~V}$ compared to the increase in theoretical conductance at about $-0.3 \mathrm{~V}$, while the right side (electron injection) increase in conductivity for both theoretical and experimental is about $0.7 \mathrm{~V}$. 
Theoretical vs. Experimental Conductance

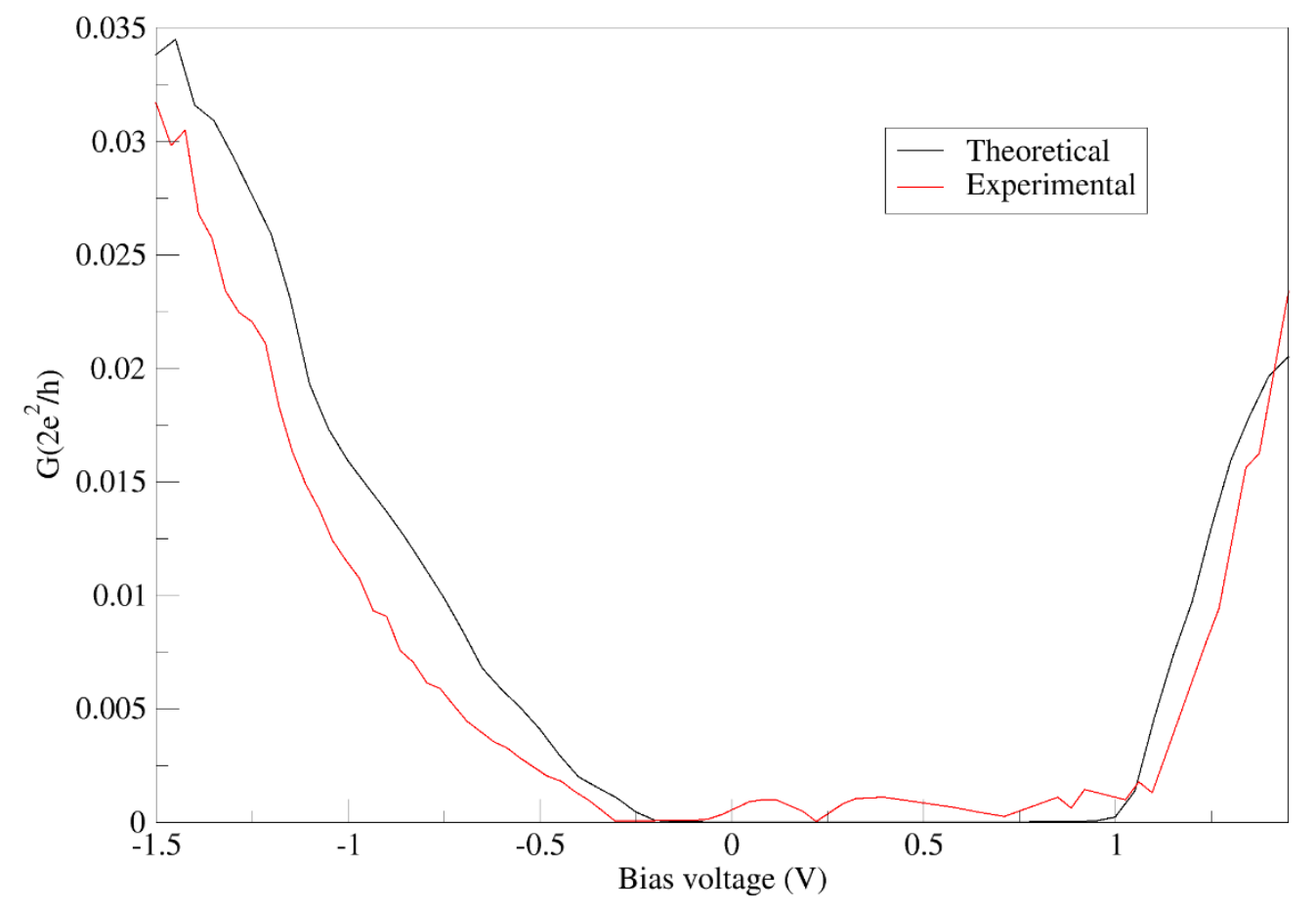

Figure 4.19: Theoretical bulk conductance adjusted for Pd contact resistance as compared to experimental conductance from $\mathrm{WSe}_{2}$ sample. 
Theoretical vs. Experimental Conductance

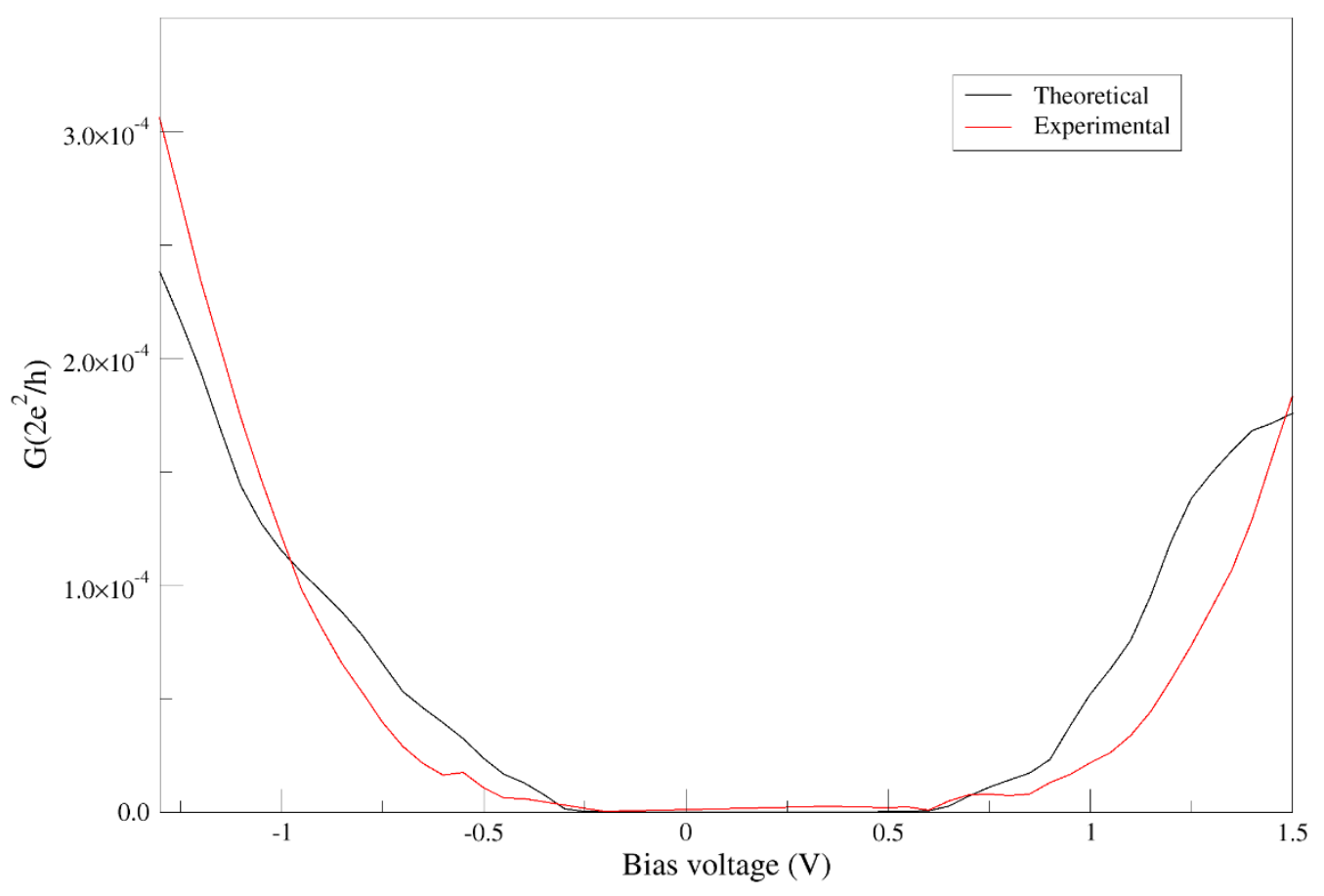

Figure 4.20: Theoretical bulk conductance adjusted for Ni contact resistance as compared to experimental conductance from $\mathrm{WSe}_{2}$ sample

There are several scattering mechanisms that can also affect the speed of the electrons and therefore conductance. The mobility at room temperature is predominately limited by phonon scattering [86]. In this mode, the electrons (or holes) make frequent collisions with the vibrating atoms of the lattice and scattering increases with increasing temperature. Another key factor impacting scattering is carrier mass. Since the electron mass is smaller than the hole mass, the electrons accelerate faster and therefore are more 
likely to scatter [6]. This is also known as lattice scattering. At low temperatures, scattering from defects and impurities are the dominating scattering mechanism, however for our devices this is unlikely since the measurements were taken at room temperature. Another scattering mechanism is surface roughness scattering which can occur at the surface of the semiconductor if it is not pristine and free of dangling bonds. This has been seen in CVD grown $\mathrm{WSe}_{2}$ films [87] due to grain boundaries that were created during the growth process. The $\mathrm{WSe}_{2}$ films grown in this research were smooth with no obvious visible grain boundaries and therefore scattering from grain boundaries is less likely for these devices. Another surface roughness scattering issue occurs when high gate fields (>1 MV/cm) drive carriers closer to the semiconductor surface causing them to scatter with dangling bonds [88]. Due to the van der Waals bonds, the surface is free of dangling bonds making the device less susceptible to this type of scattering. Considering the Schottky barrier height differences and potential effects due to possible phonon scattering, we find that conductance of the experimental device compares very well with the theoretical once the contact resistance from the experimental FET is included. Figure 4.21 shows the complete electronic band structure, quantum conductance, and density of states for a bulk layer film. 

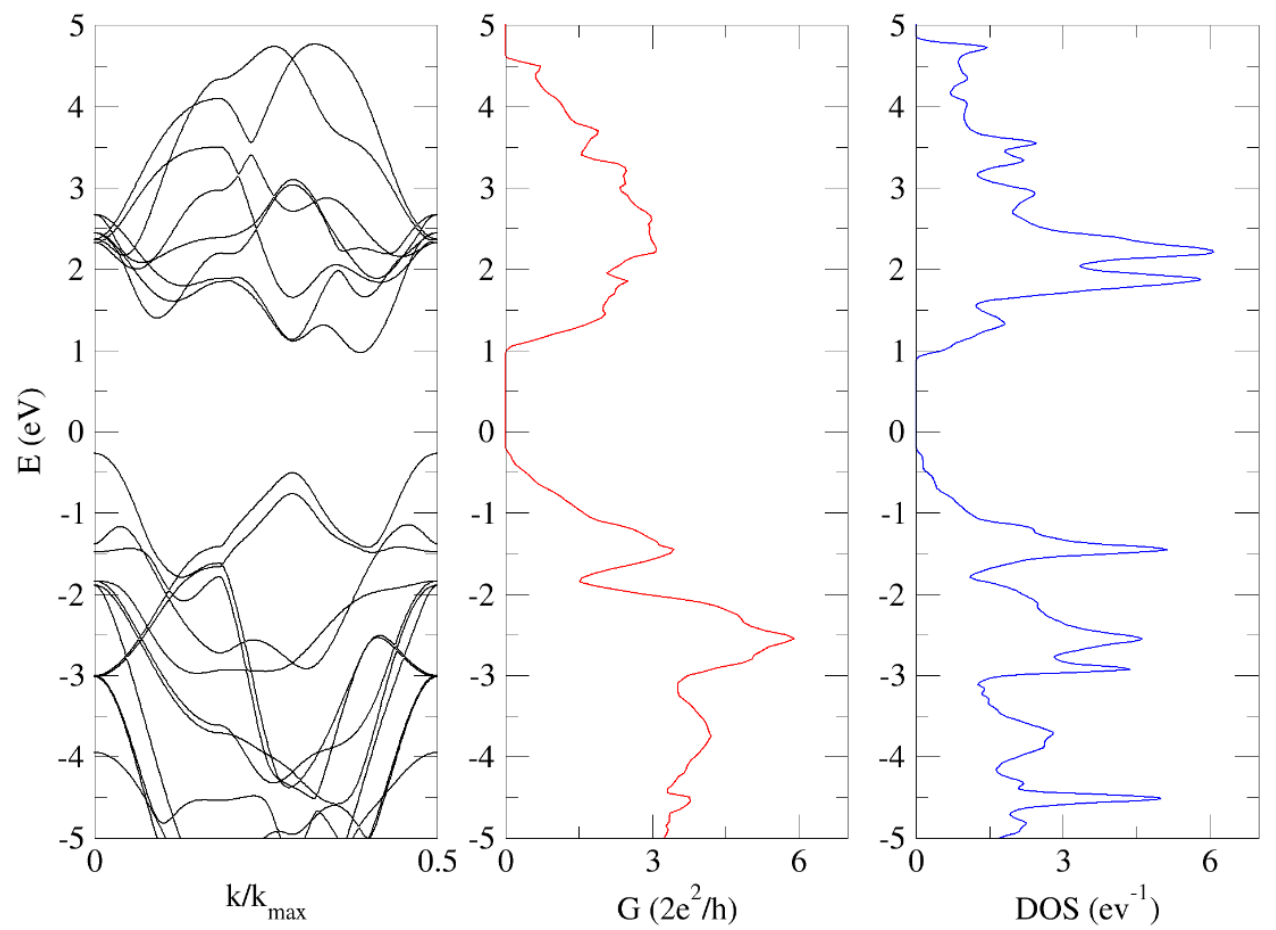

Figure 4.21: $\mathrm{WSe}_{2}$ bulk film band structure, conductance, and density for states

The electronic structure of $\mathrm{WSe}_{2}$ has been characterized by DFT methods with the relevant physics and properties reviewed. Calculations were performed to show the band gap, density of states, and the conductance. The band gap was shown to be indirect for bulk material but becomes direct band gap at the monolayer. The PDOS calculations show the main contribution to the electronic performance is due to the $d$ orbitals of W and the $p$ orbitals of Se atoms. The relative position of the $d$ orbitals plays an important 
role when choosing a contact metal. A metal with a high work function such as Pd, which has full $4 d$ shells and is near $E_{f}$, overlaps and strongly hybridizes with the valence bands of $\mathrm{WSe}_{2}$. This is what gives Pd nearly Ohmic contact as seen in figure 3.10. The theoretical conductance data matches closely with that found previously in literature [66] and closely matches the experimental data when contact resistance is accounted for.

\subsection{Summary}

Quantum mechanical modeling under the framework of density functional theory provides a very useful tool for the understanding of the nature of periodic crystal structures such as the TMDs. Electronic structure simulations based upon density functional theory is fundamental to providing a deeper understanding of the nature, as well as the predictability of these materials. The modeling performed in this research was used to show the electronic band structure of two primary materials, $\mathrm{MoS}_{2}$ and $\mathrm{WSe}_{2}$, from monolayer to bulk thickness. The band structure provided useful information about these films, such as the magnitude of the band gap and whether the material is direct or indirect. Both $\mathrm{MoS}_{2}$ and $\mathrm{WSe}_{2}$ are shown to be direct band gap semiconductors at the monolayer and indirect band gap in bulk film. The differences between band gaps of the TMDs are due to several factors, such as the size of the metal atom and energy of the metal atom $d$ orbital, the $p$ orbitals of the chalcogen atoms and bond length of the metal to chalcogen atom [89]. The projected density of states plot shows the number of available states for electrons to occupy, projected onto atomic orbitals. These plots reveal which shell (s, p, d, f) and orbital of a specified atom contributes the most to the total density of states. The data from these plots and the projected density of states shows that 
Mo and $\mathrm{W} d$ orbitals contribute to the states near the conduction band while the $\mathrm{S}$ and $\mathrm{Se}$ $p$ orbitals contribute to the states near the valence band. These factors explain why Ti with its low work function work best for $\mathrm{MoS}_{2}$ while Pd and Au with their high work functions work best for $\mathrm{WSe}_{2}$ to create Ohmic contacts. From the previous calculations, quantum conductance calculations were performed which showed good correlation to the experimental data after fitting resistance of experimental data to the theoretical values. The quantum mechanical modeling has proven to be a very valuable tool, enabling a complete and thorough understanding of the electronic structure of the TMDs. 


\section{Chapter V: Conclusions and Future Work}

\subsection{Conclusions}

The need for new materials that can replace silicon as the gate channel material is becoming clear as we continue to reduce transistor dimensions. As discussed in chapter one, TMDs are a promising family of layered materials that have the necessary properties to be used as a gate channel material. With their many remarkable electronic properties, these materials have great potential as a future option for advanced semiconductor processes. The advantages of using TMD films have been discussed, and the need to fully investigate them is clear.

Atomic layer growth of several of the transition metal dichalcogenides $\left(\mathrm{MoS}_{2}\right.$, $\mathrm{WSe}_{2}, \mathrm{WS}_{2}, \mathrm{SnS}$ and heterostructure $\mathrm{WS}_{2} / \mathrm{SnS}$ ) was demonstrated using atomic layer deposition. The physical characterizations that were performed, demonstrated that these films possess the same unique characteristics seen in literature with several other growth techniques, however we show that high quality atomically thin layers of TMD films can be grown over a large area using ALD. Most of the research into these materials is from data obtained from small flakes or small area films, which has given great insight into the properties of these materials, however to be useful for future semiconductor technology, ALD is a necessary deposition technique that must be investigated. The ability to grow these films using ALD is the key component of this research, and it has been demonstrated with several TMD films. Once these TMD films were grown, it was 
necessary to create back-gated FETs to test the electrical characteristics they possess, so that we can understand if they possess the same properties as other methods.

Electrical tests were performed that provided data for several FET devices and show key important electrical characteristics of these films. The films exhibited moderately high current on/off ratios, high field effect mobilities, photoluminescence, and direct/indirect bandgaps. The electrical data has shown that these films exhibit the necessary characteristics required to be used as a gate channel material and enable further scaling of semiconductor devices.

To better understand these films and to gain further insight into their atomic level properties, quantum mechanical modeling was performed. This study contributed to the physical and electrical data analysis by performing quantum mechanical modeling using the Quantum ESPRESSO and WanT program suites to perform electronic structure and transport calculations, and then compare those to the experimental data from devices characterized. The 2D materials analyzed during the modeling also showed the electronic structure band diagrams with the direct and indirect bandgaps of the two modeled films $\mathrm{MoS}_{2}$ and $\mathrm{WSe}_{2}$. It also showed that the band gap gets larger as the film thickness is reduced in both $\mathrm{MoS}_{2}$ and $\mathrm{WSe}_{2}$. When comparing $\mathrm{WSe}_{2}$ theoretical conductivity to the experimental conductivity from one of our devices, we see the experimental data matched very well with theoretical data once the resistance from metal contacts was applied to the theoretical. The modeling provided a nice bridge from the theoretical to the actual data and further confirms that ALD grown TMD films do indeed possess the necessary properties to be useful in advanced semiconductor material research. 
Research into TMDs is growing rapidly, even producing new journals such as $2 D$ Materials which was created specifically for publishing fundamental and applied research on graphene and related 2D materials. There is still much to learn from the study of these materials. The research that has been presented in this work resulted in three peer reviewed publications on TMDs [28, 41, 47], and several invitations for presentations.

\subsection{Future work}

One of the challenges with using TMDs as a semiconductor gate channel material is that they are intrinsically either p- or n-type. As discussed in chapter three, there is a need to be able to either dope the film, or to grow heterostructures to obtain p- or n-type

devices. One of the areas of future work should be to further investigate multiple types of heterostructures and the ability to grow them using ALD. This research focused on one combination of films in only one arrangement, but there are several films that can be combined in multiple arrangements, which are expected to have unique attributes. The ability to form heterostructures that take the positive properties of each film and apply to the combined film would be a great contribution to the field.

To achieve high performance TMD based FETs, the formation of a high- $\kappa$ gate dielectric is important. Since the surface of TMD films are pristine and free of dangling bonds, and low reactive surface sites, the formation of high- $\kappa$ gate dielectric films via ALD is difficult. Research into methods of depositing good quality thin dielectric films is an important area for future work. 
As was demonstrated in this research, low resistance contacts are difficult to form and require the right metal to minimize the Schottky barrier. Even with the appropriate low/high work function metal, Schottky barriers are inevitable and can limit high performance TMD based FETs. The ultrathin TMD films limit the traditional doping method of ion implantation used to create the low resistance contact, therefore novel doping methods need to be pursued to prevent relying on different contact metals.

There are many avenues of research into the use of TMD films as a channel material for future silicon based electronics. This research focused on ALD as a method of creating high quality films over a large area, which will be necessary for mainstream applications. However, there is much work to be accomplished if we are to be able to use TMD films in mainstream silicon based electronics. 


\section{Bibliography}

1. Dennard, R.H., et al., Design of ion-implanted MOSFET's with very small physical dimensions. Solid-State Circuits, IEEE Journal of, 1974. 9(5): p. 256-268.

2. Neamen, D., Semiconductor Physics And Devices.

3. Doyle, B.S., et al., High performance fully-depleted tri-gate CMOS transistors. IEEE Electron Device Letters, 2003. 24(4): p. 263-265.

4. Novoselov, K.S., et al., Electric Field Effect in Atomically Thin Carbon Films. Science, 2004. 306(5696): p. 666-669.

5. Frey, G.L., et al., Optical-absorption spectra of inorganic fullerenelike MS2 (M=Mo, W). Physical Review B, 1998. 57(11): p. 6666-6671.

6. Liu, W., et al., High-Performance Field-Effect-Transistors on Monolayer WSe2. 2013.

7. Gong, C., et al., The unusual mechanism of partial Fermi level pinning at metalMoS2 interfaces. Nano Lett, 2014. 14(4): p. 1714-20.

8. Amani, M., et al., Electrical performance of monolayer MoS2 field-effect transistors prepared by chemical vapor deposition. Applied Physics Letters, 2013. 102(19): p. 193107.

9. Lee, W.Y., T.M. Besmann, and M.W. Stott, Preparation of MoS2 thin films by chemical vapor deposition. Journal of Materials Research, 1994. 9(06): p. 14741483.

10. Imanishi, N., K. Kanamura, and Z.i. Takehara, Synthesis of MoS2 Thin Film by Chemical Vapor Deposition Method and Discharge Characteristics as a Cathode of the Lithium Secondary Battery. Journal of The Electrochemical Society, 1992. 139(8): p. 2082-2087.

11. Hofmann, W., Thin films of molybdenum and tungsten disulphides by metal organic chemical vapour deposition. Journal of Materials Science, 1988. 23(11): p. 3981-3986.

12. Li, Q., et al., Polycrystalline Molybdenum Disulfide (2H-MoS2) Nano- and Microribbons by Electrochemical/Chemical Synthesis. Nano Letters, 2004. 4(2): p. 277-281.

13. Zhang, Y., et al., Direct observation of the transition from indirect to direct bandgap in atomically thin epitaxial MoSe2. Nat Nano, 2014. 9(2): p. 111-115.

14. Zhou, K.G., et al., A mixed-solvent strategy for efficient exfoliation of inorganic graphene analogues. Angew Chem Int Ed Engl, 2011. 50(46): p. 10839-42.

15. Matte, H.S., et al., MoS2 and WS2 analogues of graphene. Angew Chem Int Ed Engl, 2010. 49(24): p. 4059-62.

16. Eda, G., et al., Photoluminescence from chemically exfoliated MoS2. Nano Lett, 2011. 11(12): p. 5111-6.

17. Radisavljevic, B., et al., Single-layer MoS2 transistors. Nat Nanotechnol, 2011. 6(3): p. 147-50. 
18. Zeng, Z., et al., Single-layer semiconducting nanosheets: high-yield preparation and device fabrication. Angew Chem Int Ed Engl, 2011. 50(47): p. 11093-7.

19. Coleman, J.N., et al., Two-Dimensional Nanosheets Produced by Liquid Exfoliation of Layered Materials. Science, 2011. 331(6017): p. 568-571.

20. Lee, C., et al., Anomalous Lattice Vibrations of Single- and Few-Layer MoS2. ACS Nano, 2010. 4(5): p. 2695-2700.

21. Liu, H., et al., Molecular-beam epitaxy of monolayer and bilayer WSe2: a scanning tunneling microscopy/spectroscopy study and deduction of exciton binding energy. 2D Materials, 2015. 2(3): p. 034004.

22. Jiao, L., et al., Molecular-beam epitaxy of monolayer MoSe2: growth characteristics and domain boundary formation. New Journal of Physics, 2015. 17(5): p. 053023.

23. Liu, B., et al., Chemical Vapor Deposition Growth of Monolayer WSe2 with Tunable Device Characteristics and Growth Mechanism Study. ACS Nano, 2015. 9(6): p. 6119-6127.

24. Bethge, O., et al., Low temperature atomic layer deposition of high-k dielectric stacks for scaled metal-oxide-semiconductor devices. Thin Solid Films, 2009. 517(18): p. 5543-5547.

25. Puurunen, R.L., A Short History of Atomic Layer Deposition: Tuomo Suntola's Atomic Layer Epitaxy. Chemical Vapor Deposition, 2014. 20(10-11-12): p. 332344.

26. Gordon, R.A., et al., Structures of exfoliated single layers of WS2, MoS2, and MoSe2 in aqueous suspension. Physical Review B, 2002. 65(12).

27. Tan, L.K., et al., Atomic layer deposition of a MoS2 film. Nanoscale, 2014. 6(18): p. 10584-8.

28. Browning, R., et al., Atomic layer deposition of MoS2 thin films. Materials Research Express, 2015. 2(3): p. 035006.

29. Liu, K.-K., et al., Growth of large-area and highly crystalline MoS2 thin layers on insulating substrates. Nano letters, 2012. 12(3): p. 1538-1544.

30. Li, H., et al., From Bulk to Monolayer MoS2: Evolution of Raman Scattering. Advanced Functional Materials, 2012. 22(7): p. 1385-1390.

31. Luo, X., et al., Anomalous frequency trends in MoS2 thin films attributed to surface effects. 2013.

32. Kuc, A., N. Zibouche, and T. Heine, Influence of quantum confinement on the electronic structure of the transition metal sulfideTS2. Physical Review B, 2011. 83(24).

33. Mak, K.F., et al., Atomically ThinMoS2: A New Direct-Gap Semiconductor. Physical Review Letters, 2010. 105(13).

34. Altavilla, C., M. Sarno, and P. Ciambelli, A Novel Wet Chemistry Approach for the Synthesis of Hybrid 2D Free-Floating Single or Multilayer Nanosheets of MS2@oleylamine ( $M=M o, W)$. Chemistry of Materials, 2011. 23(17): p. 38793885 . 
35. Suzuki, K., et al., Reactivity of molybdenum disulfide surfaces studied by XPS. Journal of Electron Spectroscopy and Related Phenomena, 1981. 24(2): p. 283287.

36. Goloveshkin, A.S., et al., Structural Properties and Phase Transition of ExfoliatedRestacked Molybdenum Disulfide. The Journal of Physical Chemistry C, 2013. 117(16): p. 8509-8515.

37. Zatsepin, D.A., et al., Structural ordering in a silica glass matrix under Mn ion implantation. Journal of Physics: Condensed Matter, 2012. 24(18): p. 185402.

38. Li, H., et al., Mechanical exfoliation and characterization of single-and few-layer nanosheets of WSe(2) , TaS(2), and TaSe(2). Small, 2013. 9(11): p. 1974-81.

39. Mead, D. and J. Irwin, Long wavelength optic phonons in WSe2. Canadian Journal of Physics, 1977. 55(5): p. 379-382.

40. Sahin, H., et al., Anomalous Raman spectra and thickness-dependent electronic properties of WSe2. Physical Review B, 2013. 87(16).

41. Browning, R., et al., Large area growth of layered WSe2 films. Semiconductor Science and Technology, 2016. 31(9): p. 095002.

42. Fang, H., et al., High-Performance Single Layered WSe2 p-FETs with Chemically Doped Contacts. Nano Letters, 2012. 12(7): p. 3788-3792.

43. Boscher, N.D., C.J. Carmalt, and I.P. Parkin, Atmospheric pressure chemical vapor deposition of WSe2thin films on glass-highly hydrophobic sticky surfaces. J. Mater. Chem., 2006. 16(1): p. 122-127.

44. Huang, J.-K., et al., Large-Area Synthesis of Highly Crystalline WSe2 Monolayers and Device Applications. ACS Nano, 2014. 8(1): p. 923-930.

45. Berkdemir, A., et al., Identification of individual and few layers of WS2 using Raman Spectroscopy. Scientific Reports, 2013. 3.

46. Zhao, W., et al., Lattice dynamics in mono- and few-layer sheets of WS2 and WSe2. Nanoscale, 2013. 5(20): p. 9677-83.

47. Browning, R., et al., Growth of multiple WS2/SnS layered semiconductor heterojunctions. Nanoscale, 2016.

48. Sinsermsuksakul, P., et al., Atomic Layer Deposition of Tin Monosulfide Thin Films. Advanced Energy Materials, 2011. 1(6): p. 1116-1125.

49. Chandrasekhar, H.R., et al., Infrared and Raman spectra of the IV-VI compounds SnS and SnSe. Physical Review B, 1977. 15(4): p. 2177-2183.

50. Gong, Y., et al., Vertical and in-plane heterostructures from WS2/MoS2 monolayers. Nat Mater, 2014. 13(12): p. 1135-42.

51. Radisavljevic, B. and A. Kis, Mobility engineering and a metal-insulator transition in monolayer MoS(2). Nat Mater, 2013. 12(9): p. 815-20.

52. Lembke, D. and A. Kis, Breakdown of High-Performance Monolayer MoS2 Transistors. ACS Nano, 2012. 6(11): p. 10070-10075.

53. Liu, H., A.T. Neal, and P.D. Ye, Channel length scaling of MoS2 MOSFETs. Acs Nano, 2012. 6(10): p. 8563-8569. 
54. Kang, J., et al., Computational Study of Metal Contacts to Monolayer TransitionMetal Dichalcogenide Semiconductors. Physical Review X, 2014. 4(3).

55. Popov, I., G. Seifert, and D. Tomanek, Designing electrical contacts to MoS2 monolayers: a computational study. Phys Rev Lett, 2012. 108(15): p. 156802.

56. Wu, W., et al., High mobility and high on/off ratio field-effect transistors based on chemical vapor deposited single-crystal MoS2 grains. Applied Physics Letters, 2013. 102(14): p. 142106.

57. Xiong, F., et al., Li Intercalation in MoS2: In Situ Observation of Its Dynamics and Tuning Optical and Electrical Properties. Nano Lett, 2015. 15(10): p. 6777-84.

58. Reshak, A.H. and S. Auluck, Calculated optical properties of $2 \mathrm{H}-$ MoS2intercalated with lithium. Physical Review B, 2003. 68(12).

59. Enyashin, A.N. and G. Seifert, Density-functional study of LixMoS2 intercalates $(0 \leqslant x \leqslant 1)$. Computational and Theoretical Chemistry, 2012. 999: p. 13-20.

60. Allain, A., et al., Electrical contacts to two-dimensional semiconductors. Nat Mater, 2015. 14(12): p. 1195-205.

61. Leong, W.S., C.T. Nai, and J.T. Thong, What does annealing do to metal-graphene contacts? Nano Lett, 2014. 14(7): p. 3840-7.

62. Allain, A. and A. Kis, Electron and Hole Mobilities in Single-Layer WSe2. ACS Nano, 2014. 8(7): p. 7180-7185.

63. Das, S. and J. Appenzeller, WSe2 field effect transistors with enhanced ambipolar characteristics. Applied Physics Letters, 2013. 103(10): p. 103501.

64. Pradhan, N.R., et al., Hall and field-effect mobilities in few layered $p$-WSe(2) fieldeffect transistors. Sci Rep, 2015. 5: p. 8979.

65. Zhou, H., et al., Large area growth and electrical properties of p-type WSe2 atomic layers. Nano Lett, 2015. 15(1): p. 709-13.

66. Wang, Y., et al., Does P-type Ohmic Contact Exist in WSe2-metal Interfaces? Nanoscale, 2015.

67. De, D., et al., High on/off ratio field effect transistors based on exfoliated crystalline SnS2 nano-membranes. Nanotechnology, 2013. 24(2): p. 025202.

68. Martin, R.M., Electronic Structure: Basic Theory and Practical Methods. 2004: Cambridge University Press.

69. Hohenberg, P. and W. Kohn, Inhomogeneous Electron Gas. Physical Review, 1964. 136(3B): p. B864-B871.

70. Kohn, W. and L.J. Sham, Self-Consistent Equations Including Exchange and Correlation Effects. Physical Review, 1965. 140(4A): p. A1133-A1138.

71. Perdew, J.P., K. Burke, and M. Ernzerhof, Generalized Gradient Approximation Made Simple. Physical Review Letters, 1996. 77(18): p. 3865-3868.

72. Giannozzi, P., et al., QUANTUM ESPRESSO: a modular and open-source software project for quantum simulations of materials. J Phys Condens Matter, 2009. 21(39): p. 395502.

73. Enyashin, A.N., et al., New Route for Stabilization of 1T-WS2and MoS2Phases. The Journal of Physical Chemistry C, 2011. 115(50): p. 24586-24591. 
74. Mishina, E., et al., Observation of two polytypes of MoS2 ultrathin layers studied by second harmonic generation microscopy and photoluminescence. Applied Physics Letters, 2015. 106(13): p. 131901.

75. Monkhorst, H.J. and J.D. Pack, Special points for Brillouin-zone integrations. Physical Review B, 1976. 13(12): p. 5188-5192.

76. Jiang, H., Electronic Band Structures of Molybdenum and Tungsten Dichalcogenides by the GW Approach. The Journal of Physical Chemistry C, 2012. 116(14): p. 7664-7671.

77. Enyashin, A.N. and G. Seifert, Electronic Properties of MoS2 Monolayer and Related Structures. Nanosystems: Physics, Chemistry, Mathematics, 2014. 5(4): p. 517-539.

78. Kadantsev, E.S. and P. Hawrylak, Electronic structure of a single MoS2 monolayer. Solid State Communications, 2012. 152(10): p. 909-913.

79. Kumar, A. and P.K. Ahluwalia, Electronic structure of transition metal dichalcogenides monolayers $1 H-M X 2(M=M o, W ; X=S$, Se, Te) from ab-initio theory: new direct band gap semiconductors. The European Physical Journal B, 2012. 85(6).

80. Böker, T., et al., Band structure of MoS2, MoSe2, and $\alpha-M o T e 2:$ Angle-resolved photoelectron spectroscopy and ab initio calculations. Physical Review B, 2001. 64(23).

81. Splendiani, A., et al., Emerging photoluminescence in monolayer MoS2. Nano Lett, 2010. 10(4): p. 1271-5.

82. Nardelli, M.B., Electronic transport in extended systems: Application to carbon nanotubes. Physical Review B, 1999. 60(11): p. 7828-7833.

83. Gibertini, M., G. Pizzi, and N. Marzari, Engineering polar discontinuities in honeycomb lattices. Nat Commun, 2014. 5: p. 5157.

84. Marzari, N. and D. Vanderbilt, Maximally localized generalized Wannier functions for composite energy bands. Physical Review B, 1997. 56(20): p. 12847-12865.

85. Liu, G.B., et al., Electronic structures and theoretical modelling of twodimensional group-VIB transition metal dichalcogenides. Chem Soc Rev, 2015. 44(9): p. 2643-63.

86. Schmidt, H., F. Giustiniano, and G. Eda, Electronic transport properties of transition metal dichalcogenide field-effect devices: surface and interface effects. Chem Soc Rev, 2015. 44(21): p. 7715-36.

87. Huang, J., et al., Large-area synthesis of monolayer WSe(2) on a SiO(2)/Si substrate and its device applications. Nanoscale, 2015. 7(9): p. 4193-8.

88. Jin, S., M.V. Fischetti, and T.W. Tang, Modeling of Surface-Roughness Scattering in Ultrathin-Body SOI MOSFETs. IEEE Transactions on Electron Devices, 2007. 54(9): p. 2191-2203.

89. Kang, J., et al., Band offsets and heterostructures of two-dimensional semiconductors. Applied Physics Letters, 2013. 102(1): p. 012111. 